\title{
The Aeroacoustics of Slowly Diverging Supersonic Jets
}

\author{
By M. E. Goldstein ${ }^{1}$ and S. J. Leib ${ }^{2}$ \\ ${ }^{1}$ National Aeronautics and Space Administration, Glenn Res. Center, Cleveland, $\mathrm{OH} 44135$, USA \\ ${ }^{2}$ Ohio Aerospace Institute, Brook Park, $\mathrm{OH} 44135$, USA
}

This paper is concerned with utilizing the acoustic analogy approach to predict the sound from unheated supersonic jets. Previous attempts have been unsuccessful at making such predictions over the Mach number range of practical interest. The present paper, therefore, focuses on implementing the necessary refinements needed to accomplish this objective. The important effects influencing peak supersonic noise turn out to be source convection, mean flow refraction, mean flow amplification, and source non-compactness. It appears that the last two effects have not been adequately dealt with in the literature. The first of these because the usual parallel flow models produce most of the amplification in the so called critical layer where the solution becomes singular and, therefore, causes the predicted sound field to become infinite as well. We deal with this by introducing a new weakly non parallel flow analysis that eliminates the critical layer singularity. This has a strong effect on the shape of the peak noise spectrum. The last effect places severe demands on the source models at the higher Mach numbers because the retarded time variations significantly increase the sensitivity of the radiated sound to the source structure in this case. A highly refined (non-separable) source model is, therefore, introduced in this paper.

\section{Introduction}

The impracticality of directly calculating the sound field from the full NavierStokes equations creates a need for a viable reduced order model for these equations--typically referred to as an acoustic analogy following an approach initiated by Lighthill (1952). This is analogous to the situation in turbulence modeling, which is almost always based on some form of the filtered NavierStokes equations such as the Reynolds averaged Navier-Stokes (RANS) equations. While there is some disagreement about the proper choice of turbulence modeling equations, the situation in Aeroacoustics is even more contentious. Here, there is considerable disagreement about the appropriate starting equations. Perhaps because there is no single set of equations that is optimal in all situations. There does, however, seem to be a consensus about some of the requirements for such equations. First, they should be derivable from the Navier-Stokes equations and second, they should be formally linear (Dowling \& Ffowcs Williams, 1983, p.157). 
It, therefore, makes sense to describe this phenomenon by dividing the flow variables into their mean and fluctuating components. The Navier-Stokes equations can then be rewritten as a set of mean flow equations plus a formally linear set of equations for the fluctuating component of the motion. And, it turns out that the latter can be put into the form of the linearized Navier-Stokes equations (about the mean flow) but with source terms whose strengths can be represented by a four dimensional stress tensor, whose first 3 dimensions correspond to the usual fluctuating Reynolds stress with the additional dimension being associated with the stagnation enthalpy fluctuations. The true non-linearity of these equations is hidden in the source terms as well as in the non-linearity of the dependent variables. The latter causes no particular difficulty in the present context because one of these variables (the pressure-like variable) reduces to the ordinary pressure fluctuation in the far field.

The apparent linearity can be exploited by using a Green's function approach to separate out the so called "propagation effects" from the unsteady source fluctuations, which are represented by a generalized fluctuating stress tensor in the present approach. The result can then be used to express the far field pressure autocovariance as the convolution product of a "propagator" (which can be calculated from the Green's function) with the two point timedelayed correlation of the fluctuating stress tensor--which is a statistical entity of the type that is typically measured in turbulent flows. It is also the quantity that ultimately has to be modeled in the present acoustic analogy approach. It is expected that most of the non-local "propagation effects", which would be very difficult to distinguish from the turbulent fluctuations--and, therefore, very difficult to model-- have been removed from these stresses. An attractive feature of the original Lighthill (1952) approach is that the source strength is represented by a single stress tensor, which insures that the far field pressure autocovariance can be expressed in terms of a two point time delayed correlation tensor. This significantly simplifies the interpretation of the source (Ffowcs Williams, 1963, 1969) and thereby aids in the construction of appropriate models for this quantity. The present result (which is similar to the one in Goldstein (2003)) also has this important property which, together with the two requirements set out at the beginning of this section, effectively restricts the form of the analogy to relatively trivial rearrangements of a single set of equations.

Current state of the art noise prediction methodologies, typically, use empirical models for the unknown Reynolds stress correlations and RANS based approaches (typically of the k-epsilon type) to calculate the mean flow and mean turbulent kinetic energy. The former is then used to calculate the coefficients in the Green's function equations, while the latter is used to determine the parameters empirical models for the fluctuating Reynolds stresses.

This approach is very general and should, in principle, apply to any turbulent flow. But jet flows are nearly parallel, and the mean flow is usually approximated by a much simpler unidirectional transversely sheared flow. The resulting acoustic equations can then be reduced to a single $3^{\text {rd }}$ order equation frequently referred to as Lilley's (Lilley, 1972, 1974) equation. 
This type of model appears to be adequate for subsonic, unheated jets (Khavaran \& Bridges, 2004) but becomes unphysical at supersonic acoustic Mach numbers where it predicts infinite acoustic pressures. The difficulty is due to the breakdown of the parallel flow model and can be eliminated by retaining the correct non-parallel mean flow. But this would greatly increase the complexity of the Green's function computation.

The present paper introduces a perturbation approach that takes advantage of the small jet spread rate. It uses matched asymptotic expansions to obtain a locally parallel "outer solution" that applies in the main part of the jet where the parallel flow "propagator" is non-singular and a non-singular "inner solution" that accounts for the nonparallel flow effects in the vicinity of the so called critical layer where the outer (parallel flow) solution breaks down. The results are then combined to obtain a uniformly valid "composite" solution that produces physically reasonable results and is still not much more complicated than the parallel flow solution. The resulting "propagator" still becomes large when the source point is at the critical layer but, unlike the parallel flow result, remains finite there.

A similar weakly non-parallel flow approach was used by Goldstein and Leib (2005) to construct a causal Green's function that remains finite in the small spread rate limit. It results in an additional contribution to the usual parallel flow Green's function that involves the linear instability modes of the jet. It was incorrectly stated in that paper that the result, which did not account for the critical layer singularity, was uniformly valid everywhere in the flow. The complete uniformly valid causal solution can only be obtained by combining the solution in the present paper with the Goldstein and Leib (2005) result. But this would be extremely complicated and our most recent computations show that the instability wave contribution tends to be very small at the relatively low acoustic Mach numbers being considered in the paper. We, therefore, decided to neglect that contribution (in the manner discussed in section 5) in order to focus on the critical-layer effects without introducing undue complication.

While strong streamwise and relatively weak transverse coherence effects preclude the possibility of assuming that the sources are completely compact (i.e., of neglecting all variations in retarded time across the sources), it is still highly desirable to model the situation as closely as possible. The relevant source model should, therefore, account for streamwise variations in retarded time and be general enough to account for the long range turbulence correlations at the end of the potential core which, in particular, precludes using such assumptions as local isotropy and quasi-normality. The model should also be simple enough to produce easily computed expressions for the spectral tensor components. The present paper introduces such a model. Its most general form involves a large number of adjustable constants, which can be parameterized and subsequently determined from an auxiliary flow calculation. Current state of the art noise prediction codes, such as JeNo (Khavaran \& Bridges, 2004), usually use steady $\mathrm{k}-\varepsilon$ based computations for this purpose. But codes of this type only provide enough information to determine a small number of these parameters. It 
is, however, highly likely that higher fidelity methods such as full Reynolds Stress Modeling or even hybrid RANS / Large Eddy Simulation approaches will replace the steady k- $\varepsilon$ computations. These codes can provide enough information to determine many more of the adjustable constants.

Previous attempts -typically based on much simpler flow models-have not been successful at predicting jet noise over the parameter range of practical interest. They are usually unable to adequately predict the sound radiation from heated jets or the peak radiation from all supersonic jets. This has even caused some writers (see for example, Viswanathan, 2007) to recommend that the acoustic analogy approach be abandoned in favor of empirical correlations of the jet noise data base. But this approach is unlikely to be very robust beyond the parameter range of the available data. The present paper, therefore, focuses on implementing the necessary refinements into the acoustic analogy. It attempts to demonstrate that good predictions can be obtained when a high level of rigor is maintained by minimizing the approximations and keeping the formulas exact until the last stages of the analysis in order to make the required approximations as consistent as possible. We have chosen to not consider heated jets because the available computer codes for producing required information about the flow have not been adequately calibrated with an appropriate experimental data base. The focus is, therefore, on unheated supersonic jets, but the general formulas are applicable to heated jets as well.

The important effects influencing peak supersonic noise turn out to be source convection, mean flow refraction, mean flow amplification, and source non-compactness. It is our contention that the last two effects have not been adequately dealt with in the literature. The first of these because, as originally noted by Phillips (1960), the usual parallel flow (i.e. Lilley equation) models produce most of the amplification in the so called critical layer where the solution becomes singular and causes the predicted sound field to become infinite. We deal with this by introducing a new weakly non parallel flow analysis that eliminates the critical layer singularity. This has a strong effect on the shape of the peak noise spectrum. The inability of previous attempts to deal with the last effect can be traced to inadequate source models. Retarded time variations can significantly increase the sensitivity of the radiated sound to the detailed source structure. A much more refined (non-separable) source model is, therefore, developed in section 6.2 of this paper.

The overall plan of the paper is as follows: First, the basic acoustic analogy equations are set out in section 2 and a formal Green's function solution is written down in section 3 . The resulting expression for the pressure-like variable is an integral of a tensor product of a "propagator" that depends only on the mean flow variables, with a generalized four dimensional stress tensor that completely characterizes the turbulent velocity/enthalpy fluctuations. The propagator depends on the Green's function for an arbitrary mean flow. The latter is simplified in section 4 by assuming that the mean flow is weakly non-parallel. It is shown that the strictly parallel flow result breaks down in the so called critical layer and matched asymptotic expansions have to be used to obtain a uniformly valid (i.e. non-singular) result. 
The Green's function solution of section 3 is used in section 5 to obtain an expression for the far field pressure autocovariance in terms of a two-point time delayed correlation of the turbulent velocity/ enthalpy fluctuations. This exact result, which shows that the far field pressure autocovariance can be expressed as a convolution product of a new propagator with this generalized correlation tensor, is the fundamental starting equation of this paper. It is used to obtain an expression for the far field acoustic spectrum in terms of the weakly non-parallel flow Green's function constructed in section 4, which is then simplified by assuming that the transverse correlation length of the turbulence was small compared to the transverse length scale of the jet. The resulting expression for the acoustic spectrum is now purely algebraic and involves a product of a propagator and a generalized spectral tensor that accounts for the turbulent velocity/enthalpy fluctuations. It is only at this point that the results are restricted to "cold" jets by neglecting the enthalpy fluctuations.

The propagation tensor is completely characterized by the mean flow which, with the present approach, is to be calculated from a RANS solution. But the generalized spectral tensor requires detailed information about the turbulence statistics, which can not be directly obtained from the steady RANS solution. It, therefore, has to be modeled, which is done in section 6. First "kinematic models" which exploit expected symmetry properties of the turbulence correlation functions, are introduced in section 6.1 to reduce its large number of independent components (45 in all, even if the enthalpy fluctuations are neglected). Then a very general correlation function model is introduced in section 6.2 to represent the spectra of the surviving components of this tensor. It involves a large number of parameters that can be related to the output of the RANS solution by making appropriate modeling assumptions. But before doing this, the general results are specialized to a round jet in section 7 . The modeling assumptions are introduced in section 8 and the results are then compared with detailed jet noise measurements taken over a number of years at the Glenn Research Center.

\section{Basic Equations}

Assume that the pressure $p$, density $\rho$, enthalpy $h$, speed of sound $c$ and absolute temperature $T$ satisfy the ideal gas law equation of state

$$
p=\rho R T, \quad h=c_{p} T=c_{v} c^{2} / R
$$

with $R=c_{p}-c_{v}$ being the gas constant, and $c_{p}$ and $c_{v}$ the specific heats at constant pressure and volume, respectively. Then the Navier-Stokes equations can be written as

$$
\begin{gathered}
D \rho=0 \\
D\left(\rho v_{i}\right)+\frac{\partial p}{\partial x_{i}}=\frac{\partial}{\partial x_{j}} \sigma_{i j}
\end{gathered}
$$




$$
D\left(\frac{\gamma p}{\gamma-1}+\frac{\rho}{2} v^{2}\right)-\frac{\partial p}{\partial t}=\frac{\partial}{\partial x_{i}}\left(\frac{\sigma_{4 i}}{\gamma-1}+\sigma_{i j} v_{j}\right)
$$

where $i, j=1,2,3$, the operator $D$ is defined by

$$
\mathrm{D} f \equiv \frac{\partial f}{\partial t}+\frac{\partial}{\partial x_{j}}\left(v_{j} f\right)
$$

for any function $f, t$ denotes the time, $\boldsymbol{x}=\left\{x_{1}, x_{2}, x_{3}\right\}$ the Cartesian coordinates, $\boldsymbol{v}=\left\{v_{1}, v_{2}, v_{3}\right\}$ the fluid velocity. $\sigma_{i j}$ denotes the viscous stress, $\sigma_{4 i} \equiv-(\gamma-1) q_{i}$, the scaled heat flux vector, and $\gamma \equiv c_{p} / c_{v}$ the specific heat ratio.

By introducing the new pressure like variable

$$
\bar{p}_{e} \equiv \bar{p}+\frac{\gamma-1}{2} \widetilde{\rho} \widetilde{v^{\prime 2}}
$$

the Favre (1969) averaged Navier-Stokes (RANS) equations can be written in the virtually identical form

$$
\begin{gathered}
D_{o} \bar{\rho}=0 \\
D_{o}\left(\bar{\rho} \tilde{v}_{i}\right)+\frac{\partial \bar{p}_{e}}{\partial x_{i}}=\frac{\partial}{\partial x_{j}} \overline{e_{i j}^{\prime}} \\
D_{o}\left(\frac{\gamma \bar{p}_{e}}{\gamma-1}+\frac{\bar{\rho}}{2} \tilde{v}^{2}\right)-\frac{\partial \bar{p}_{e}}{\partial t}=\frac{\partial}{\partial x_{i}}\left(\frac{\overline{e_{4 i}^{\prime}}}{\gamma-1}+\bar{e}_{i j}^{\prime} \tilde{v}_{j}\right)
\end{gathered}
$$

where

$$
D_{o} f \equiv \frac{\partial f}{\partial t}+\frac{\partial}{\partial x_{j}}\left(\tilde{v}_{j} f\right)
$$

for any function $f$, the over bars denote the usual time average

$$
\bar{\bullet} \equiv \lim _{T \rightarrow \infty} \frac{1}{2 T} \int_{-T}^{T} \bullet(\boldsymbol{x}, t) d t
$$

with the dot being a place holder for $\rho, v_{i}, p, h$,etc. and 


$$
\widetilde{\bullet} \equiv(\overline{\rho \bullet} / \bar{\rho})
$$

denotes a Favre (1969) average, it being understood that the time derivatives drop out of the RANS equations (2.7) to (2.9),

$$
e_{v i}^{\prime} \equiv-\rho v_{v}^{\prime} v_{i}^{\prime}+\delta_{v i} \frac{(\gamma-1)}{2} \rho v^{\prime 2}+\left[\sigma_{v i}+(\gamma-1) \delta_{v 4} \sigma_{i k} v_{k}^{\prime}\right]
$$

with $v=1,2,3,4$,

$$
v_{4}^{\prime} \equiv(\gamma-1)\left(h^{\prime}+\frac{1}{2} v^{\prime 2}\right)=\left(c^{2}\right)^{\prime}+\frac{(\gamma-1)}{2} v^{\prime 2}
$$

and $\delta_{v j}$ denoting the Kronecker delta in the usual notation.

The primes denote the fluctuating variables

$$
\rho^{\prime} \equiv \rho-\bar{\rho}, \quad p^{\prime} \equiv p-\bar{p}, \quad h^{\prime} \equiv h-\tilde{h}, \quad v_{i}^{\prime} \equiv v_{i}-\tilde{v}_{i}
$$

etc., which upon introducing the (non-linear) dependent variables

$$
\begin{gathered}
p_{e}^{\prime} \equiv p^{\prime}+\frac{\gamma-1}{2}\left(\rho v^{\prime 2}-\overline{\rho v^{\prime 2}}\right) \\
\mathrm{u}_{\mathrm{i}} \equiv \rho \mathrm{v}_{\mathrm{i}}^{\prime}
\end{gathered}
$$

can be shown to satisfy the five formally linear equations (Goldstein, 2003)

$$
\begin{gathered}
D_{o} \rho^{\prime}+\frac{\partial}{\partial x_{j}} u_{j}=0 \\
D_{o} u_{i}+u_{j} \frac{\partial \tilde{v}_{i}}{\partial x_{j}}+\frac{\partial}{\partial x_{i}} p_{e}^{\prime}-\frac{\rho^{\prime}}{\bar{\rho}} \frac{\partial}{\partial x_{j}} \tilde{\theta}_{i j}=\frac{\partial}{\partial x_{j}} e_{i j}^{\prime \prime}
\end{gathered}
$$

and

$$
D_{o} p_{e}^{\prime}+\frac{\partial}{\partial x_{j}} \widetilde{c^{2}} u_{j}+(\gamma-1)\left(p_{e}^{\prime} \frac{\partial \tilde{v}_{j}}{\partial x_{j}}-\frac{u_{i}}{\bar{\rho}} \frac{\partial \tilde{\theta}_{i j}}{\partial x_{j}}\right)=\frac{\partial}{\partial x_{j}} e_{4 j}^{\prime \prime}+(\gamma-1) e_{i j}^{\prime \prime} \frac{\partial \tilde{v}_{i}}{\partial x_{j}}
$$

where 


$$
\tilde{\theta}_{i j} \equiv \delta_{i j} \bar{p}_{e}-\overline{e_{i j}^{\prime}},
$$

is the total mean flow stress tensor,

$$
\widetilde{c^{2}} \equiv \gamma \bar{p} / \bar{\rho}
$$

the square of the mean-flow sound speed, and the source strengths $e_{v j}^{\prime \prime}, v=1,2,3,4$, which are given by

$$
e_{\mu j}^{\prime \prime} \equiv e_{\mu j}^{\prime}-\overline{e_{\mu j}^{\prime}},
$$

have zero time average.

This shows that the entire acoustic source strength is given by the generalized (fluctuating velocity-sound speed) stress tensor $e_{v j}^{\prime \prime}, v=1,2,3,4$-which is an important advantage of the present formulation because it puts all of the modeling on the same basis and thereby reduces the probability of over/under estimating one component of the source relative to another due to differences in modeling requirements (see paragraph following equation(5.29)).

Notice that (2.7) to (2.9) will constitute a closed system of five equations in the five unknowns $\bar{\rho}, \tilde{v}_{i}, \bar{p}_{e}$ that can be solved independently of the relation (2.6) between the effective pressure $\bar{p}_{e}$ and the thermodynamic pressure $\bar{p}$ once a particular turbulence model has been introduced. This is also true for the linear system (2.18) to (2.20) in the variables $\rho^{\prime}, u_{i}, p_{e}^{\prime}$---with their nonlinear relation to the physical variables $\rho^{\prime}, v_{i}, p^{\prime}$ being largely irrelevant because the variable $p_{e}^{\prime}$ reduces to the acoustic pressure fluctuation $p^{\prime}$ in the far field where the sound field is to be calculated.

\section{Formal Green's Function Solution for the Far Field Pressure}

The particular solution to equations (2.18) to (2.20) can, as noted in the introduction, be expressed in terms of the vector Green's function (Morse and Feshbach 1953, pp. 878-886) $g_{v \sigma}(\boldsymbol{x}, t \mid \boldsymbol{y}, \tau)$, which satisfies

$$
\begin{gathered}
D_{0} g_{i \sigma}+g_{j \sigma} \frac{\partial \tilde{v}_{i}}{\partial x_{j}}+\frac{\partial g_{4 \sigma}}{\partial x_{i}}-\frac{1}{\bar{\rho}} \frac{\partial \tilde{\theta}_{i j}}{\partial x_{j}} g_{5 \sigma}=\delta_{i \sigma} \delta(\boldsymbol{x}-\boldsymbol{y}) \delta(t-\tau) \\
D_{0} g_{4 \sigma}+(\gamma-1)\left(\frac{\partial \tilde{v}_{i}}{\partial x_{i}} g_{4 \sigma}-\frac{1}{\bar{\rho}} \frac{\partial \tilde{\theta}_{i j}}{\partial x_{j}} g_{i \sigma}\right)+\frac{\partial \widetilde{c^{2}} g_{j \sigma}}{\partial x_{j}}=\delta_{4 \sigma} \delta(\boldsymbol{x}-\boldsymbol{y}) \delta(t-\tau)
\end{gathered}
$$




$$
D_{o} g_{5 \sigma}+\frac{\partial}{\partial x_{j}} g_{j \sigma}=0
$$

for $\sigma=1,2, \ldots, 5$ and can be inserted into Green's formula to obtain the following expression for the pressure like variable $p_{e}^{\prime}$ (Goldstein,2006)

$$
p_{e}^{\prime}(\boldsymbol{x}, t)=-\int_{V} \int_{-\infty}^{\infty} \gamma_{\mu j}(\boldsymbol{x}, t \mid \boldsymbol{y}, \tau) e_{\mu j}^{\prime \prime}(\boldsymbol{y}, \tau) d \mathbf{y} d \tau
$$

where $V$ denotes integration over all space ,the propagator $\gamma_{\mu j}(\boldsymbol{x}, t \mid \boldsymbol{y}, \tau)$ is defined by

$$
\gamma_{\mu j}(\boldsymbol{x}, t \mid \boldsymbol{y}, \tau) \equiv \frac{\partial g_{4 \mu}(\boldsymbol{x}, t \mid \boldsymbol{y}, \tau)}{\partial y_{j}}-(\gamma-1) \frac{\partial \tilde{v}_{\mu}}{\partial y_{j}} g_{44}(\boldsymbol{x}, t \mid \boldsymbol{y}, \tau)
$$

$\tilde{v}_{4} \equiv 0$, and we have integrated by parts to transfer the derivatives from the source term to $g_{4 v}(\boldsymbol{x}, t \mid \boldsymbol{y}, \tau)$.

\section{Weakly Non-Parallel Flow Approximation}

As noted in the Introduction, the propagator $\gamma_{\mu j}(\boldsymbol{y}, \tau \mid \boldsymbol{x}, t)$ can be greatly simplified by assuming that the mean flow is locally parallel. In fact, Appendix A, which describes the mean flow perturbation expansion, shows that equations (3.1) to (3.3) reduce to the inhomogeneous Rayleigh equations (i.e., the equations governing the inviscid instability of a parallel flow) in this limit

$$
\begin{gathered}
\frac{D_{o}}{D t} g_{i \sigma}+\frac{\partial}{\partial x_{i}} g_{4 \sigma}+\delta_{i 1} \frac{\partial U}{\partial x_{j}} g_{j \sigma}=\delta_{i \sigma} \delta(\boldsymbol{x}-\boldsymbol{y}) \delta(t-\tau), \\
\frac{D_{o}}{D t} g_{4 \sigma}+\frac{\partial}{\partial x_{i}}\left(\widetilde{c_{0}^{2}} g_{i \sigma}\right)=\delta_{4 \sigma} \delta(\boldsymbol{x}-\boldsymbol{y}) \delta(t-\tau), \\
\frac{D_{o}}{D t} g_{5 \sigma}+\frac{\partial}{\partial x_{j}} g_{j \sigma}=0,
\end{gathered}
$$

where

$$
\frac{D_{0}}{D t}=\frac{\partial}{\partial t}+U \frac{\partial}{\partial x_{1}}
$$


is the usual convective derivative. Since the solution to this system will depend on $x_{1}, y_{1}, t, \tau$ only in the combinations $x_{1}-y_{1}$ and $t-\tau$, we put

$$
\begin{aligned}
& \hat{g}_{\mathrm{v \sigma}}\left(\boldsymbol{x}_{T} \mid \boldsymbol{y}_{T} ; k, \omega\right) \\
& \equiv \frac{1}{(2 \pi)^{2}} \iint e^{-i\left[k\left(x_{1}-y_{1}\right)-\omega(t-\tau)\right]} g_{v \sigma}(\boldsymbol{x}, t \mid \boldsymbol{y}, \tau) d(t-\tau) d\left(x_{1}-y_{1}\right),
\end{aligned}
$$

where $\boldsymbol{x}_{T}=\left\{x_{2,} x_{3}\right\}$ and $\boldsymbol{y}_{T}=\left\{y_{2,} y_{3}\right\}$ denote the transverse components of $\boldsymbol{x}$ and $\boldsymbol{y}$ respectively. The resulting equation for $\hat{g}_{4 \sigma}\left(\boldsymbol{x}_{T} \mid \boldsymbol{y}_{T} ; k, \omega\right)$ has a regular singular point at the so called critical layer where

$$
\omega-k U\left(\boldsymbol{x}_{T}\right)=0,
$$

and the solution has an infinite singularity there, which (as will be shown below) produces a much stronger singularity in the propagator $\bar{\gamma}_{v j \mu l}$ (defined below). The original singularity in $\hat{g}_{v \sigma}\left(\boldsymbol{x}_{T} \mid \boldsymbol{y}_{T} ; k, \omega\right)$ can be eliminated by constructing an 'inner solution' in the vicinity of the critical layer which has continuous pressure and normal velocity components but discontinuous tangential velocity components (i.e., it has a slip line). However, we do not present the details here because our interest is in the corresponding singularity that occurs in the source variable $\boldsymbol{y}$ when the observation point $\boldsymbol{x}$ is in the far field. This singularity can only be found by working with the $4^{\text {th }}$ component of the adjoint vector Green's function

$$
g_{\sigma v}^{a}(\boldsymbol{y}, \tau \mid \boldsymbol{x}, t)=g_{v \sigma}(\boldsymbol{x}, t \mid \boldsymbol{y}, \tau)
$$

which (unlike the direct Green's function that is determined by equations in the observation variables $\boldsymbol{x}, t)$ is determined by the following set of equations in the source variables $\boldsymbol{y}, \tau$ (Morse and Feshbach (1953), p.870; Goldstein (2006))

$$
\begin{gathered}
-\frac{\tilde{D} g_{i 4}^{a}}{D \tau}+g_{j 4}^{a} \frac{\partial \tilde{v}_{j}}{\partial y_{i}}-\widetilde{c^{2}} \frac{\partial g_{44}^{a}}{\partial y_{i}}-\frac{\gamma-1}{\bar{\rho}} \frac{\partial \tilde{\theta}_{i j}}{\partial y_{j}} g_{44}^{a}-\frac{\partial g_{54}^{a}}{\partial y_{i}}=0 \\
-\frac{\tilde{D} g_{44}^{a}}{D \tau}-\frac{\partial g_{i 4}^{a}}{\partial y_{i}}+(\gamma-1) g_{44}^{a} \frac{\partial \tilde{v}_{j}}{\partial y_{j}}=\delta(\boldsymbol{x}-\boldsymbol{y}) \delta(t-\tau)
\end{gathered}
$$




$$
-\frac{\tilde{D} g_{54}^{a}}{D \tau}-\frac{1}{\bar{\rho}} \frac{\partial \tilde{\theta}_{i j}}{\partial y_{j}} g_{i 4}^{a}=0
$$

where

$$
\frac{\tilde{D}}{D \tau} \equiv \frac{\partial}{\partial \tau}+\tilde{v}_{i}(\boldsymbol{y}) \frac{\partial}{\partial y_{i}}
$$

Using Appendix A and retaining only terms $O(\varepsilon)$ leads to

$$
\begin{gathered}
-\frac{D_{0} g_{i 4}^{a}}{D \tau}+g_{14}^{a} \frac{\partial U}{\partial y_{i}}-\widetilde{c^{2}} \frac{\partial g_{44}^{a}}{\partial y_{i}}-\frac{\partial g_{54}^{a}}{\partial y_{i}}=\varepsilon\left[\left(U \frac{\partial}{\partial Y}+U^{(1)} \frac{\partial}{\partial y_{1}}+V_{j} \frac{\partial}{\partial y_{j}}\right) g_{i 4}^{a}\right. \\
\left.-g_{j 4}^{a} \frac{\partial V_{j}}{\partial y_{i}}-g_{14}^{a}\left(\frac{\partial U^{(1)}}{\partial y_{i}}+\delta_{i 1} \frac{\partial U}{\partial Y}\right)+\delta_{i 1}\left(\widetilde{c^{2}} \frac{\partial g_{44}^{a}}{\partial Y}-\frac{\gamma-1}{\bar{R}} S g_{44}^{a}+\frac{\partial g_{54}^{a}}{\partial Y}\right)\right] \\
-\frac{D_{0} g_{44}^{a}}{D \tau}-\frac{\partial g_{i 4}^{a}}{\partial y_{i}}=\delta(\boldsymbol{x}-\boldsymbol{y}) \delta(t-\tau)+\varepsilon\left[\left(U \frac{\partial}{\partial Y}+U^{(1)} \frac{\partial}{\partial y_{1}}+V_{j} \frac{\partial}{\partial y_{j}}\right) g_{44}^{a}+\frac{\partial g_{14}^{a}}{\partial Y}\right. \\
\left.-(\gamma-1) g_{44}^{a}\left(\frac{\partial V_{j}}{\partial y_{j}}+\frac{\partial U}{\partial Y}\right)\right] \\
-\frac{D_{0} g_{54}^{a}}{D \tau}=\varepsilon\left[\left(U \frac{\partial}{\partial Y}+U^{(1)} \frac{\partial}{\partial y_{1}}+V_{j} \frac{\partial}{\partial y_{j}}\right) g_{54}^{a}-\frac{S}{\bar{R}} g_{14}^{a}\right]
\end{gathered}
$$

where $j=2,3$ and $D_{0} / D \tau$ is defined by (4.4).

\subsection{The 'Outer Solution'}

Equations (4.12) to (4.14) suggest that $g_{v \mu}^{a}$ should expand like 


$$
g_{v \mu}^{a}=g_{v \mu}^{a, 0}+\varepsilon g_{v \mu}^{a, 1}+\ldots
$$

in any region where it is nonsingular and that the lowest order term $g_{v \mu}^{a, 0}$ can be expressed in terms of the single scalar Green's function $G^{a}(\boldsymbol{y}, \tau \mid \boldsymbol{x}, t)$ by

$$
g_{i 4}^{a, 0}=-\widetilde{c^{2}}\left(\frac{\partial}{\partial y_{i}} \frac{D_{0}}{D \tau}+2 \frac{\partial U}{\partial y_{i}} \frac{\partial}{\partial y_{1}}\right) G^{a}, \quad g_{44}^{a, 0}=\frac{D_{0}^{2} G^{a}}{D \tau^{2}}
$$

which satisfies the $3^{\text {rd }}$ order equation

$$
\left(\frac{\partial}{\partial y_{i}} \widetilde{c^{2}} \frac{\partial}{\partial y_{i}} \frac{D_{0} G^{a}}{D \tau}\right)-\frac{D_{0}^{3} G^{a}}{D \tau^{3}}+2 \frac{\partial}{\partial y_{j}}\left(\frac{\partial U}{\partial y_{j}} \widetilde{c^{2}} \frac{\partial}{\partial y_{1}} G^{a}\right)=\delta(\boldsymbol{x}-\boldsymbol{y}) \delta(t-\tau)
$$

in this region.

Since the coefficients in (4.12) to (4.14) are independent of time and only depend on $y_{1}$ through the slow streamwise variable $Y$ its solution should depend on $x_{1}, y_{1}, t$ and $\tau$ only in the combinations $x_{1}-y_{1}$ and $t-\tau$. And it, therefore, makes sense to put

$$
\begin{aligned}
& \hat{g}_{\mathrm{v} 4}^{a}\left(\boldsymbol{y}_{T}, Y \mid \boldsymbol{x}_{T} ; k, \omega, \varepsilon\right) \\
& \equiv \frac{1}{(2 \pi)^{2}} \iint e^{-i\left[k\left(x_{1}-y_{1}\right)-\omega(t-\tau)\right]} g_{\mathrm{v} 4}^{a}(\boldsymbol{y}, \tau \mid \boldsymbol{x}, t ; \varepsilon) d(t-\tau) d\left(x_{1}-y_{1}\right)
\end{aligned}
$$

It then follows from (4.16) that

$$
\left.\begin{array}{c}
\hat{g}_{i 4}^{a, 0}=-\widetilde{c^{2}} i\left[\frac{\partial}{\partial y_{i}}(\omega-U k)-2 k \frac{\partial U}{\partial y_{i}}\right] \hat{G}^{a}=\frac{\widetilde{c^{2}}}{(\omega-U k)^{2}} \frac{\partial \hat{G}_{0}}{\partial y_{i}}, \mathrm{i}=2,3 \\
\hat{g}_{14}^{a, 0}=-\widetilde{c^{2}} k(\omega-U k) \hat{G}^{a}=\frac{-i k \widetilde{c^{2}}}{(\omega-U k)^{2}} \hat{G}_{0} \\
\hat{g}_{44}^{a, 0}=-(\omega-U k)^{2} \hat{G}^{a}=\frac{-i}{(\omega-U k)} \hat{G}_{0}
\end{array}\right\}
$$

where

$$
\hat{G}_{0}\left(\boldsymbol{y}_{T}, Y \mid \boldsymbol{x}_{T} ; k, \omega\right) \equiv
$$




$$
\begin{array}{r}
-i \frac{\left(\omega-U\left(\boldsymbol{y}_{T}\right) k\right)^{3}}{(2 \pi)^{2}} \iint e^{-i\left[k\left(x_{1}-y_{1}\right)-\omega(t-\tau)\right]} G^{a}(\boldsymbol{y}, \tau \mid \boldsymbol{x}, t) d(t-\tau) d\left(x_{1}-y_{1}\right) \\
\equiv-i\left(\omega-U\left(\boldsymbol{y}_{T}\right) k\right)^{3} \hat{G}^{a}\left(\boldsymbol{y}_{T}, Y \mid \boldsymbol{x}_{T} ; k, \omega\right)
\end{array}
$$

Is determined by

$$
\begin{gathered}
\widetilde{\mathcal{L}_{k}} \hat{G}_{o}=\frac{\delta\left(\boldsymbol{x}_{T}-\boldsymbol{y}_{T}\right)}{(2 \pi)^{2}} \\
\widetilde{\mathcal{L}_{k}} \equiv \frac{\partial}{\partial y_{j}} \frac{\widetilde{c^{2}}}{(k U-\omega)^{2}} \frac{\partial}{\partial y_{j}}+1-\frac{k^{2} \widetilde{c^{2}}}{(k U-\omega)^{2}} \quad j=2,3
\end{gathered}
$$

(Afsar et al (2006) show that this formulation is computationally advantageous when the mean flow is only known at a discrete number of points)Then, corresponding to (4.15)

$\hat{g}_{v \sigma}^{a}\left(\boldsymbol{y}_{T}, Y \mid \boldsymbol{x}_{T} ; k, \omega, \varepsilon\right)=\hat{g}_{v \sigma}^{a, 0}\left(\boldsymbol{y}_{T}, Y \mid \boldsymbol{x}_{T} ; k, \omega\right)+\varepsilon \hat{g}_{v \sigma}^{a, 1}\left(\boldsymbol{y}_{T}, Y \mid \boldsymbol{x}_{T} ; k, \omega, \varepsilon\right)+\ldots$

in the region where $\hat{g}_{v \mu}^{a}$ is nonsingular, and the leading order term satisfies

$$
\begin{gathered}
-i(\omega-U k) \hat{g}_{14}^{a, 0}+i k\left(\widetilde{c^{2}} \hat{g}_{44}^{a, 0}+\hat{g}_{54}^{a, 0}\right)=0 \\
-i(\omega-U k) \hat{g}_{i 4}^{a, 0}+\hat{g}_{14}^{a, 0} \frac{\partial U}{\partial y_{i}}-\widetilde{c^{2}} \frac{\partial \hat{g}_{44}^{a, 0}}{\partial y_{i}}-\frac{\partial \hat{g}_{54}^{a, 0}}{\partial y_{i}}=0, \text { for } i=2,3 \\
-i(\omega-U k) \hat{g}_{44}^{a, 0}-\frac{\partial \hat{g}_{i 4}^{a, 0}}{\partial y_{i}}+i k \hat{g}_{14}^{a, 0}=\frac{1}{(2 \pi)^{2}} \delta\left(\boldsymbol{x}_{T}-\boldsymbol{y}_{T}\right) \\
i(\omega-U k) \hat{g}_{54}^{a, 0}=0
\end{gathered}
$$

there. The fifth component, $\hat{g}_{54}^{a, 0}$, which is determined independently of the remaining components by (4.25), is indeterminate at this stage of the analysis. These equations also have a regular singular point in the vicinity of the critical layer which passes through the line of singular points, say $\boldsymbol{y}_{T}=\boldsymbol{y}_{c}$, where 


$$
\omega-k U\left(\boldsymbol{y}_{c}\right)=0 .
$$

The resulting solution will exhibit a strong infinite singularity when the source variable $\boldsymbol{y}_{T}$ approaches this point, which can be attributed to the breakdown of the parallel flow model. It can be eliminated by constructing a local non-parallel flow solution in the vicinity of this point--referred to here as the "inner solution". The critical layer, which disappears when the source variable $\boldsymbol{y}_{c}$ moves onto the jet centerline, will lie on the level curve/surface of $U$, say $S_{c}$, where the dominant balance of the outer (parallel flow) solution turns out to be (Wundrow, \& Goldstein 1994)

$$
\begin{aligned}
\hat{g}_{\sigma 4}^{a, 0} & =\frac{\hat{a}_{\sigma}\left(s, Y \mid \boldsymbol{x}_{T}\right)}{y_{\perp}^{2}}+\hat{b}_{\sigma}\left(s, Y \mid \boldsymbol{x}_{T}\right)+\hat{c}_{\sigma}\left(s, Y \mid \boldsymbol{x}_{T}\right) y_{\perp} \ln y_{\perp}+\ldots, \quad \sigma=1, t \\
\hat{g}_{\perp 4}^{a, 0}= & \frac{\hat{b}_{\perp 4}\left(s, Y \mid \boldsymbol{x}_{T}\right)}{y_{\perp}}+\hat{c}_{\perp 4}\left(s, Y \mid \boldsymbol{x}_{T}\right) \ln y_{\perp}+\ldots, \\
\hat{g}_{44}^{a, 0} & =\frac{\hat{a}_{4}\left(s, Y \mid \boldsymbol{x}_{T}\right)}{y_{\perp}}+\hat{b}_{4}\left(s, Y \mid \boldsymbol{x}_{T}\right) y_{\perp}+\hat{c}_{4}\left(s, Y \mid \boldsymbol{x}_{T}\right) y_{\perp}^{2} \ln y_{\perp}+\ldots, \\
\hat{g}_{54}^{a, 0} & =\ldots \ldots \ldots
\end{aligned}
$$

where the $\hat{a}_{v}, \hat{b}_{v}, \hat{c}_{v} \ldots$ are completely determined by the outer solution while the dots in the last equation are meant to reflect the indeterminacy of $\hat{g}_{54}^{a, 0}$ at this point in the analysis, $s$ denotes the transverse distance along $S_{c}$ and $y_{\perp} \equiv\left(\boldsymbol{y}_{T}-\boldsymbol{y}_{\boldsymbol{c}}\right) \cdot \hat{\boldsymbol{n}}$ denotes the perpendicular distance to this surface, i.e., the distance in the direction of its unit normal $\hat{\boldsymbol{n}}$ (see figure 1 ).

\subsection{The 'Inner Solution'}

The solution in the 'inner region' $\bar{y}_{\perp} \equiv y_{\perp} / \delta=\left(\boldsymbol{y}_{T}-\boldsymbol{y}_{\boldsymbol{c}}\right) \cdot \hat{\boldsymbol{n}} / \delta=O(1)$, where $\delta$ denotes an appropriate scale factor for the critical layer, should therefore expand like

$$
\begin{aligned}
& \hat{g}_{\sigma 4}^{a}=\delta^{-2} g_{\sigma 4}^{0}\left(\bar{y}_{\perp}, s, Y\right)+\delta^{-1} g_{\sigma 4}^{1}\left(\bar{y}_{\perp}, s, Y\right)+g_{\sigma 4}^{2}\left(\bar{y}_{\perp}, s, Y\right)+\ldots, \quad \sigma=1, t \\
& \hat{g}_{\perp 4}^{a}=\delta^{-1} g_{\perp 4}^{0}\left(\bar{y}_{\perp}, s, Y\right)+\ln \delta g_{\perp 4}^{1}\left(\bar{y}_{\perp}, s, Y\right)+g_{\perp 4}^{2}\left(\bar{y}_{\perp}, s, Y\right)+\delta g_{\perp 4}^{3}\left(\bar{y}_{\perp}, s, Y\right)+\ldots,
\end{aligned}
$$




$$
\hat{g}_{v 4}^{a}=\delta^{-1} g_{v 4}^{0}\left(\bar{y}_{\perp}, s, Y\right)+g_{v 4}^{1}\left(\bar{y}_{\perp}, s, Y\right)+\delta g_{v 4}^{2}\left(\bar{y}_{\perp}, s, Y\right)+\ldots, \quad v=4,5
$$

Then, since the distinguished limit corresponds to $\delta=\sqrt{\varepsilon}$,

$$
h_{s} \equiv\left|\frac{\partial \boldsymbol{y}_{T}}{\partial s}\right|=1+\delta h_{s c}^{\prime}(s) \bar{y}_{\perp}+\frac{1}{2} \delta^{2} h_{s c}^{\prime \prime}(s) \bar{y}_{\perp}^{2}+\ldots
$$

and, for any function $f=f\left(\bar{y}_{\perp}, s, Y\right)$, the chain rule implies that

$$
\left(\frac{\partial f}{\partial Y}\right)_{\mathbf{y}_{\mathrm{T}}}=\left(\frac{\partial f}{\partial Y}\right)_{\bar{y}_{\perp}, S}+\frac{\partial f}{\partial \bar{y}_{\perp}} \frac{\partial \bar{y}_{\perp}}{\partial Y}=\left(\frac{\partial f}{\partial Y}\right)_{\bar{y}_{\perp}, S}-\frac{1}{\delta} \frac{\partial f}{\partial \bar{y}_{\perp}}\left[\frac{\partial y_{c}}{\partial Y}+O(\varepsilon)\right]
$$

where $y_{c} \equiv \mathbf{y}_{c} \cdot \hat{\mathbf{n}}$, the subscript $c$ indicates that the quantity is to be evaluated at $\boldsymbol{y}_{\boldsymbol{c}}$ and the primes denote differentiation with respect to $y_{\perp}$, the lowest order terms in these expansions should satisfy

$$
\begin{gathered}
g_{14}^{0} U_{c}^{\prime}-\widetilde{c_{c}^{2}} \frac{\partial g_{44}^{0}}{\partial \bar{y}_{\perp}}-\frac{\partial g_{54}^{0}}{\partial \bar{y}_{\perp}}=0 \\
i k U_{c}^{\prime} \bar{y}_{\perp} g_{14}^{0}+i k\left(\widetilde{c_{c}^{2}} g_{44}^{0}+g_{54}^{0}\right)=\left(V_{c}-U_{c} \frac{\partial y_{c}}{\partial Y}\right) \frac{\partial}{\partial \bar{y}_{\perp}} g_{14}^{0}, \\
i k U_{c}^{\prime} \bar{y}_{\perp} g_{t 4}^{0}-\frac{\partial}{\partial s}\left(\widetilde{c_{c}^{2}} g_{44}^{0}+g_{54}^{0}\right)=\left(V_{c}-U_{c} \frac{\partial y_{c}}{\partial Y}\right) \frac{\partial}{\partial \bar{y}_{\perp}} g_{t 4}^{0}, \\
i k U_{c}^{\prime} \bar{y}_{\perp} g_{54}^{0}=\left(V_{c}-U_{c} \frac{\partial y_{c}}{\partial Y}\right) \frac{\partial}{\partial \bar{y}_{\perp}} g_{54}^{0}-\left[U_{c}\left(\frac{\partial U}{\partial Y}\right)_{c}^{0}+\frac{1}{h_{S}}\left(\frac{1}{h_{\perp}} \frac{\partial h_{\perp} g_{t 4}^{0}}{\partial s}+\frac{\partial h_{S} g_{\perp 4}^{0}}{\partial \bar{y}_{\perp}}\right)=0\right. \\
\frac{\partial h_{S} g_{\perp 4}^{1}}{\partial \bar{y}_{\perp}}=0 \\
g_{14}^{0},
\end{gathered}
$$




$$
\begin{gathered}
i k g_{14}^{1}-\frac{1}{h_{S}}\left(\frac{1}{h_{\perp}} \frac{\partial h_{\perp} g_{t 4}^{1}}{\partial s}+\frac{\partial h_{S} g_{\perp 4}^{2}}{\partial \bar{y}_{\perp}}\right)=0 \\
i k U_{c}^{\prime} \bar{y}_{\perp} g_{44}^{0}+i k g_{14}^{2}-\frac{1}{h_{S}}\left(\frac{1}{h_{\perp}} \frac{\partial h_{\perp} g_{t 4}^{2}}{\partial s}+\frac{\partial h_{S} g_{\perp 4}^{3}}{\partial \bar{y}_{\perp}}\right)=\left(V_{c}-U_{c} \frac{\partial y_{c}}{\partial Y}\right) \frac{\partial}{\partial \bar{y}_{\perp}} g_{44}^{0}+\frac{\partial g_{14}^{0}}{\partial Y},
\end{gathered}
$$

where $V_{c} \equiv \boldsymbol{V}\left(\boldsymbol{y}_{c}, Y\right) \cdot \hat{\boldsymbol{n}}$,

$$
h_{\perp} \equiv\left|\left(\frac{\partial \boldsymbol{y}_{T}}{\partial U}\right)_{S}\right| .=h_{\perp c}(s)+\delta h_{\perp c}^{\prime}(s) \bar{y}_{\perp}+\frac{1}{2} \delta^{2} h_{\perp c}^{\prime \prime}(s) \bar{y}_{\perp}^{2}+\ldots
$$

These results were obtained by using (A.12), (A.13) and (A.16) to get (4.35), and retaining higher order terms in equations (4.34) and (4.36) to (4.38)in order to circumvent the complexity of using the expansions (4.29) and (4.39).

The first of these equations, which can be written as

$$
g_{14}^{0} U_{c}^{\prime}=\frac{\partial \bar{\gamma}}{\partial \bar{y}_{\perp}},
$$

where

$$
\bar{\gamma} \equiv \widetilde{c_{c}^{2}} g_{44}^{0}+g_{54}^{0},
$$

can be used to eliminate $g_{14}^{0}$ in the $2^{\text {nd }}$ equation to obtain

$$
\frac{\partial^{2}}{\partial \bar{y}_{\perp}^{2}} \bar{\gamma}-2 \alpha^{2}\left(\bar{y}_{\perp} \frac{\partial \bar{\gamma}}{\partial \bar{y}_{\perp}}+\bar{\gamma}\right)=0
$$

where

$$
\alpha^{2} \equiv i k \frac{U_{c}^{\prime}}{2\left(V_{c}-U_{c} \frac{\partial y_{c}}{\partial Y}\right)}
$$

The solution to these equations that matches onto the $1^{\text {st }}$ and last of equations (4.27) is 


$$
\bar{\gamma}=2 \alpha^{2} \hat{a}_{4} \widetilde{c_{c}^{2}} \exp \cdot\left(\alpha^{2} \bar{y}_{\perp}^{2}\right) \int_{\bar{y}_{\perp}}^{\infty} \exp \cdot\left(-\alpha^{2} \eta^{2}\right) d \eta
$$

and

$$
g_{14}^{0}=\frac{2 \alpha^{2} \widetilde{c_{c}^{2}}}{U_{c}^{\prime}} \hat{a}_{4} \frac{\partial}{\partial \bar{y}_{\perp}} \exp \cdot\left(\alpha^{2} \bar{y}_{\perp}^{2}\right) \int_{\bar{y}_{\perp}}^{\infty} \exp \cdot\left(-\alpha^{2} \eta^{2}\right) d \eta
$$

These results, along with those of Appendix $B$ which contains the details of the critical layer expansion, show that the inner expansion for the Fourier Transform $\hat{g}_{v 4}^{a}\left(\boldsymbol{y}_{T}, Y \mid \boldsymbol{x}_{T} ; k, \omega, \varepsilon\right)$ of adjoint vector Green's function $g_{\sigma v}^{a}(\boldsymbol{y}, \tau \mid \boldsymbol{x}, t)$ can be summarized as follows:

$$
\begin{aligned}
& \widetilde{c_{c}^{2}} \hat{g}_{44}^{a}+\hat{g}_{54}^{a}=\frac{2 \alpha \widetilde{c}_{c}^{2}}{\sqrt{\varepsilon}} w\left(\alpha \bar{y}_{\perp}\right)+O(\sqrt{\varepsilon}) \\
& \hat{g}_{54}^{a}=\frac{\widetilde{c_{c}^{2}} \hat{a}_{4}}{2 \alpha \sqrt{\varepsilon}} \frac{d^{2}}{d \bar{y}_{\perp}^{2}} w\left(\alpha \bar{y}_{\perp}\right) \\
& \hat{g}_{14}^{a}=\frac{2 \alpha \widetilde{c_{c}^{2}} \hat{a}_{4}}{\varepsilon U_{c}^{\prime}} \frac{d}{d \bar{y}_{\perp}} w\left(\alpha \bar{y}_{\perp}\right)+O(1) \\
& \hat{g}_{t 4}=-\frac{2 \alpha}{\varepsilon i k U_{c}^{\prime}} \frac{\partial \widetilde{c_{c}^{2}} \hat{a}_{4}}{\partial s} \frac{d}{d \bar{y}_{\perp}} w\left(\alpha \bar{y}_{\perp}\right)+O(1) \\
& \hat{g}_{\perp 4}=-\frac{2 \alpha}{\sqrt{\varepsilon} i k U_{c}^{\prime}}\left(\frac{1}{h_{\perp}} \frac{\partial}{\partial s} h_{\perp} \frac{\partial \widetilde{c_{c}^{2}} \hat{a}_{4}}{\partial s}+k^{2} \widetilde{c_{c}^{2}} \hat{a}_{4}\right) w\left(\alpha \bar{y}_{\perp}\right) \\
& \left(-i k+\frac{\partial}{\partial Y}\right) \hat{g}_{14}^{a}+\frac{1}{h_{S}}\left(\frac{1}{h_{\perp}} \frac{\partial h_{\perp} \hat{g}_{t 4}^{a}}{\partial s}+\frac{\partial h_{S} \hat{g}_{\perp 4}^{a}}{\partial y_{\perp}}\right)=\frac{1}{\widetilde{c_{c}^{2}}}\left(V_{c}-U_{c} \frac{\partial y_{c}}{\partial Y}\right)\left[\left(2 \alpha^{2} \bar{y}_{\perp}-\frac{\partial}{\partial \bar{y}_{\perp}}\right) \bar{\gamma}+U_{c}^{\prime} g_{14}^{0}\right] \\
& =\hat{a}\left[1+\frac{1}{\alpha} \frac{d}{d \bar{y}_{\perp}} w\left(\alpha \bar{y}_{\perp}\right)\right]
\end{aligned}
$$


where equations (4.34) to (4.38) and (B.3) were used to obtain (4.51) and we have put (Abramowitz \& Stegun, 1965, p.297)

$$
\begin{aligned}
w\left(\alpha \bar{y}_{\perp}\right) \equiv & \alpha \exp .\left(\alpha^{2} \bar{y}_{\perp}^{2}\right) \int_{\bar{y}_{\perp}}^{\infty} \exp .\left(-\alpha^{2} \eta^{2}\right) d \eta= \\
& \frac{\sqrt{\pi}}{2} e^{\left(\alpha \bar{y}_{\perp}\right)^{2}} \operatorname{erfc}\left(\alpha \bar{y}_{\perp}\right) \rightarrow \frac{1}{2 \alpha \bar{y}_{\perp}}+\frac{1}{4 \alpha^{3} \bar{y}_{\perp}^{3}}+\ldots \text { as } \alpha \bar{y}_{\perp} \rightarrow \infty
\end{aligned}
$$

and

$$
\hat{a} \equiv i k U_{c}^{\prime} \hat{a}_{4} .
$$

Corresponding to(4.27), $\hat{G}_{0}$ should behave like

$$
\hat{G}_{0} \sim \hat{a}(\boldsymbol{x}, Y, s)+\hat{b}(\boldsymbol{x}, Y, s) y_{\perp}^{2}+\hat{c}(\boldsymbol{x}, Y, s) y_{\perp}^{3} \ln y_{\perp}+\ldots \text { as } y_{\perp} \rightarrow 0
$$

where $\hat{a}=\left.\hat{G}_{0}\right|_{y_{\perp}=0}$ is defined by (4.53) and

$$
\hat{\mathrm{b}}=-\left(\frac{1}{h_{\perp} \widetilde{c_{c}^{2}}} \frac{\partial}{\partial s} h_{\perp} \frac{\partial \widetilde{c_{c}^{2}} \bar{a}_{4}}{\partial s}+k^{2} \bar{a}_{4}\right)
$$

We are ultimately interested in using these results to obtain a uniformly valid expression for the space-time Fourier Transform of propagator (3.5) which, in view of(4.7) an (4.18), will depend on $\hat{g}_{\mathrm{v} 4}^{a}\left(\boldsymbol{y}_{T}, Y \mid \boldsymbol{x}_{T} ; k, \omega, \varepsilon\right)$ and its derivatives with respect to $\boldsymbol{y}_{T}$ (see(5.14)-(5.17) below). This will be accomplished by using the product rule (i.e., multiplying the inner and outer solutions and dividing the result by their common part in the overlap domain (Van Dyke,1975) to combine the inner and outer expansions for $\hat{g}_{\mathrm{v} 4}^{a}\left(\boldsymbol{y}_{T}, Y \mid \boldsymbol{x}_{T} ; k, \omega, \varepsilon\right)$. The most singular contributions come from the derivatives $\partial \hat{g}_{i 4}^{a} / \partial y_{j}, j=2,3 ; \mathrm{i} \neq \mathrm{j}$ with a typical term being $\partial \hat{g}_{14}^{a} / \partial y_{\perp}$. Equations (4.19) and (4.48) show that the inner and outer expansions for this quantity are given by $-i k \partial\left[\widetilde{c^{2}} \hat{G}_{0} /(\omega-U k)\right] / \partial y_{\perp}$ and $\hat{g}_{14}^{a}=\left(2 \alpha c_{c}^{2} \hat{a}_{4} / \varepsilon \delta U_{c}^{\prime}\right) d^{2} w\left(\alpha \bar{y}_{\perp}\right) / d \bar{y}_{\perp}^{2}$ and equations (4.52) and (4.54) show that they will match in some overlap domain where $y_{\perp} \ll 1$ and $\bar{y}_{\perp} \gg 1$. It therefore follows from the product rule that 


$$
\left(\partial \hat{g}_{14}^{a} / \partial y_{\perp}\right)_{\text {uniformly valid }}=-i k\left[\frac{\partial}{\partial y_{\perp}} \frac{\widetilde{c^{2}} \bar{G}_{0}}{(\omega-U k)}\right]\left[\frac{d^{2}}{d \bar{y}_{\perp}^{2}} w\left(\alpha \bar{y}_{\perp}\right)\right] \alpha \bar{y}_{\perp}^{2}
$$

is a uniformly valid approximation to $\partial \hat{g}_{14}^{a} / \partial y_{\perp}$ that remains bounded at $y_{\perp}=0$ and reduces to the appropriate inner and outer solutions within their respective domains of validity.

\section{The Far Field Spectrum}

The focus of this paper is on predicting the acoustic spectrum

$$
I_{\omega}(\boldsymbol{x}) \equiv \frac{1}{2 \pi} \int_{-\infty}^{\infty} e^{i \omega t} \overline{p^{2}}(\boldsymbol{x}, t) d t
$$

which is just the Fourier transform of the far-field pressure autocovariance defined by

$$
\overline{p^{2}}\left(\boldsymbol{x}, t_{0}\right) \equiv \frac{1}{2 T} \int_{-T}^{T} p^{\prime}(\boldsymbol{x}, t) p^{\prime}\left(\boldsymbol{x}, t+t_{0}\right) d t,
$$

where $T$ denotes some large, but finite, time interval. (In most cases, capital letters will be used to denote Fourier transforms of the corresponding lower case quantities) Then, since $p_{e}^{\prime} \rightarrow p^{\prime}$ as $x \rightarrow \infty$, equation (3.4) can be inserted into(5.2), and the integration variables changed to $\mathrm{t}_{1} \equiv \mathrm{t}-\tau_{2}$ and $\tau_{1} \equiv \tau_{2}-\tau$, to obtain

$\overline{p^{2}}\left(\boldsymbol{x}, t_{0}\right) \equiv$

$\frac{1}{2 T} \int_{-T}^{T} \iint_{-\infty}^{\infty} \iint_{V} \gamma_{v j}\left(\boldsymbol{x} \mid \boldsymbol{y}, t+t_{o}-\tau\right) \gamma_{\mu l}\left(\boldsymbol{x} \mid \boldsymbol{y}_{1}, t-\tau_{2}\right) e_{v j}^{\prime \prime}(\boldsymbol{y}, \tau) \mathrm{e}_{\mu l}^{\prime \prime}\left(\boldsymbol{y}_{1}, \tau_{2}\right) d \boldsymbol{y} d \boldsymbol{y}_{1} d \tau d \tau_{2} d t$

$=\iint_{-\infty}^{\infty} \iint_{V} \gamma_{v j}\left(\boldsymbol{x} \mid \boldsymbol{y}, t_{l}+t_{o}+\tau_{l}\right) \gamma_{\mu l}\left(\boldsymbol{x} \mid \boldsymbol{y}_{1}, t_{l}\right) \boldsymbol{R}_{v j \mu l}\left(\boldsymbol{y} ; \boldsymbol{y}_{1}-\boldsymbol{y}, \tau_{l}\right) d \boldsymbol{y} d \boldsymbol{y}_{l} d t_{1} d \tau_{l}$,

where we have taken the liberty of using the same symbol to denote slightly different functions and put 


$$
\begin{gathered}
\gamma_{v j}(\boldsymbol{x} \mid \boldsymbol{y}, t-\tau) \equiv \gamma_{v j}(\boldsymbol{x}, t \mid \boldsymbol{y}, \tau), \\
\boldsymbol{R}_{v j \mu l}(\boldsymbol{y} ; \boldsymbol{\eta}, \tau) \equiv \frac{1}{2 T} \int_{-T}^{T} e_{v j}^{\prime \prime}\left(\boldsymbol{y}, \tau_{0}\right) e_{\mu l}^{\prime \prime}\left(\boldsymbol{y}+\boldsymbol{\eta}, \tau_{0}+\tau\right) d \tau_{0}
\end{gathered}
$$

is the modified fixed frame density-weighted, fourth-order, two-point, timedelayed fluctuating velocity correlation and

$$
\eta \equiv y_{1}-\boldsymbol{y}
$$

denotes the separation vector between the points. This result can be put into a more transparent form by introducing a new propagator $\bar{\gamma}_{v j \mu l}$, defined in terms of the original propagator $\gamma_{j \mu}(\boldsymbol{x}, t \mid \boldsymbol{y}, \tau)$ by

$$
\bar{\gamma}_{v j \mu l}(\boldsymbol{x} \mid \boldsymbol{y} ; \boldsymbol{\eta}, t+\tau) \equiv \int_{-\infty}^{\infty} \gamma_{v j}\left(\boldsymbol{x} \mid \boldsymbol{y}, t_{1}+t+\tau\right) \gamma_{\mu l}\left(\boldsymbol{x} \mid \boldsymbol{y}+\boldsymbol{\eta}, t_{1}\right) d t_{1}
$$

to obtain the following, more compact, expression

$$
\overline{p^{2}}(\boldsymbol{x}, t)=\int_{-\infty}^{\infty} \iint_{V} \bar{\gamma}_{v j \mu l}(\boldsymbol{x} \mid \boldsymbol{y} ; \boldsymbol{\eta}, t+\tau) \boldsymbol{R}_{v j \mu l}(\boldsymbol{y} ; \boldsymbol{\eta}, \tau) d \boldsymbol{y} d \boldsymbol{\eta} d \tau
$$

for the far-field pressure autocovariance in terms of the four-dimensional twopoint, time-delayed momentum/enthalpy flux correlation tensor $\boldsymbol{R}_{v j \mu l}(\boldsymbol{y} ; \boldsymbol{\eta}, \tau)$.

This result is essentially exact, but the source correlation can contain purely convective, and therefore non-radiating, components. In an effort to minimize such components (in the Lighthill equation context), Ffowcs Williams (1963) introduced a moving frame correlation tensor which, with the present formulation, is appropriately defined by

$$
\mathbb{R}_{v j \mu l}^{M}(\boldsymbol{y} ; \xi, \tau) \equiv \boldsymbol{R}_{v j \mu l}\left(\boldsymbol{y} ; \xi+\hat{\mathbf{i}} U_{c} \tau, \tau\right),
$$

where

$$
\xi \equiv \boldsymbol{\eta}-\hat{\mathbf{i}} U_{c} \tau
$$

denotes a moving frame coordinate system, with $U_{c}$ being the "convection velocity" of the turbulence. And since this objective is consistent with the philosophy of this paper (as outlined in the Introduction), we insert (5.9)into (5.8) to obtain 


$$
\overline{p^{2}}(\boldsymbol{x}, t)=\int_{-\infty}^{\infty} \iint_{V} \bar{\gamma}_{v j \mu l}\left(\boldsymbol{x} \mid \boldsymbol{y} ; \boldsymbol{\xi}+\hat{\mathbf{i}} U_{c} \tau, t+\tau\right) \boldsymbol{R}_{v j \mu l}^{M}(\boldsymbol{y} ; \boldsymbol{\xi}, \tau) d \boldsymbol{y} d \boldsymbol{\xi} d \tau
$$

The modified fixed-frame correlation $\boldsymbol{R}_{v j \mu l}(\boldsymbol{y} ; \boldsymbol{\eta}, \tau)$ is related to the autocovariance of the generalized velocity/enthalpy Reynolds stress tensor

$$
R_{v j \mu l}(\boldsymbol{y} ; \boldsymbol{\eta}, \tau) \equiv \frac{1}{2 T} \int_{-T}^{T}\left[\rho v_{v}^{\prime} v_{j}^{\prime}-\overline{\rho v_{v}^{\prime} v_{j}^{\prime}}\right]\left(\boldsymbol{y}, \tau_{0}\right)\left[\rho v_{\mu}^{\prime} v_{l}^{\prime}-\overline{\rho v_{\mu}^{\prime} v_{l}^{\prime}}\right]\left(\boldsymbol{y}+\boldsymbol{\eta}, \tau_{0}+\tau\right) d \tau_{0}
$$

(which is about as close as you can get to what is actually measured in turbulent flows) by the simple linear transform

$$
R_{v j \mu l}=R_{v j \mu l}-\frac{\gamma-1}{2}\left(\delta_{v j} R_{k k \mu l}+\delta_{\mu l} R_{v j k k}\right)+\left(\frac{\gamma-1}{2}\right)^{2} \delta_{v j} \delta_{\mu l} R_{i i k k},
$$

with an identical relation holding between the corresponding moving frame correlation tensors $\mathbb{R}_{v j \mu l}^{M}(\boldsymbol{y} ; \boldsymbol{\xi}, \tau)$ and $R_{v j \mu l}^{M}(\boldsymbol{y} ; \boldsymbol{\xi}, \tau)$.

The far-field spectrum can be calculated by taking the Fourier transform in time of (5.11) and noting that (5.7) is of convolution form (Morse and Feshbach, 1953, p.465) to obtain

$$
I_{\omega}(\boldsymbol{x} \mid \boldsymbol{y})=2 \pi \int_{-\infty}^{\infty} \int_{V} \Gamma_{v j}(\boldsymbol{x} \mid \boldsymbol{y} ; \omega) \Gamma_{\mu l}^{*}\left(\boldsymbol{x} \mid \boldsymbol{y}+\boldsymbol{\xi}+\hat{\boldsymbol{i}} U_{c} \tau ; \omega\right) e^{-i \omega \tau} \boldsymbol{R}_{v j \mu l}^{M}(\boldsymbol{y}, \boldsymbol{\xi}, \tau) d \xi d \tau
$$

where

$$
\Gamma_{v j} \equiv \frac{1}{2 \pi} \int_{-\infty}^{\infty} e^{i \omega(t-\tau)} \gamma_{v j}(\boldsymbol{x} \mid \boldsymbol{y}, t-\tau) d(t-\tau)
$$

is the Fourier transform of $\gamma_{v j}$ and we have introduced $I_{\omega}(\boldsymbol{x} \mid \boldsymbol{y})$, the acoustic spectrum at $\mathbf{x}$ due to a unit volume of turbulence at $\mathbf{y}$, i.e.,

$$
I_{\omega}(\boldsymbol{x})=\int_{V} I_{\omega}(\boldsymbol{x} \mid \boldsymbol{y}) d \boldsymbol{y}
$$

in order to make the formula more transparent.

Appendix A and equations(3.5),(4.7),(4.18) and(5.15) imply that

$$
\Gamma_{v j}(\boldsymbol{x} \mid \boldsymbol{y} ; \omega) \equiv \int_{-\infty}^{\infty}\left[\frac{\partial}{\partial y_{j}} e^{i k\left(x_{1}-y_{1}\right)} \hat{g}_{\mathrm{v} 4}^{a}\left(\boldsymbol{y}_{T}, Y \mid \boldsymbol{x}_{T} ; k, \omega, \varepsilon\right)\right.
$$




$$
\left.-(\gamma-1) \delta_{v 1} e^{i k\left(x_{1}-y_{1}\right)} \frac{\partial U\left(Y, \boldsymbol{y}_{\boldsymbol{T}}\right)}{\partial y_{j}} \hat{g}_{44}^{a}\left(\boldsymbol{y}_{T}, Y \mid \boldsymbol{x}_{T} ; k, \omega, \varepsilon\right)\right] d k
$$

to lowest order of approximation, where the integration contour would have to be deformed below the real axis in order to pick up the contribution from the instability waves if the Green's function were required to be causal (Goldstein \& Leib, 2005). But our most recent studies suggest that this contribution should be fairly small for cold (i.e., unheated) jets at the relatively low supersonic Mach numbers being considered here. Then, since our focus is on jets of this type, we tentatively neglect it by requiring that the integration contour lie along the real axis. It can easily be incorporated, especially if it is assumed that it is uncorrelated with the non-causal contribution.

The result is much simpler in the far field where $U\left(\mathbf{x}_{T}\right) \rightarrow 0, \widetilde{c^{2}} \rightarrow c_{\infty}^{2}=$ constant, and

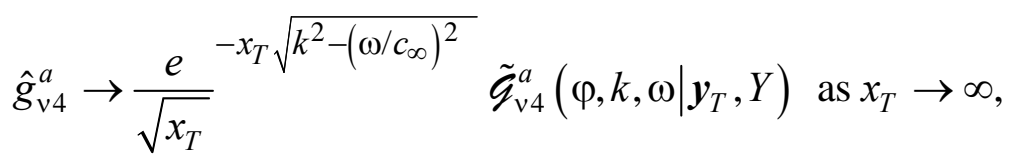

where we have used (4.5),(4.7),(4.18)and (4.21) (with $y_{j}$ replaced by $\left.x_{j}\right), x_{T} \equiv\left|\mathbf{x}_{T}\right|$ and $\varphi \equiv \tan ^{-1} x_{2} / x_{3}$ denotes the circumferential angle. Inserting this into equation(5.17), and using stationary phase to evaluate the integrals, shows that

$$
\begin{aligned}
& \Gamma_{v j} \rightarrow-\frac{e^{i \omega\left(x-y_{1} \cos \theta\right) / c_{\infty}}}{x} \sqrt{\frac{2 \pi i \omega \sin \theta}{c_{\infty}}} \bar{\Gamma}_{v j}\left(\boldsymbol{x} \mid \boldsymbol{y}_{\perp}, Y\right) \equiv \\
& -\frac{e^{i \omega x / c_{\infty}}}{x} \sqrt{\frac{2 \pi i \omega \sin \theta}{c_{\infty}}}\left\{\frac{\partial}{\partial y_{j}} \tilde{\boldsymbol{q}}_{v 4}^{a}\left(\varphi, \frac{\omega}{c_{\infty}} \cos \theta, \omega \mid \boldsymbol{y}_{T}, Y\right)\right. \\
& \left.-(\gamma-1) \delta_{v 1} \frac{\partial U\left(Y, \boldsymbol{y}_{\boldsymbol{T}}\right)}{\partial y_{j}} \tilde{\boldsymbol{q}}_{44}^{a}\left(\varphi, \frac{\omega}{c_{\infty}} \cos \theta, \omega \mid \boldsymbol{y}_{T}, Y\right)\right\} e^{-i \omega y_{1} \cos \theta / c_{\infty}}
\end{aligned}
$$

where $x \equiv|x|, \theta \equiv \sin ^{-1}\left(x_{T} / x\right)$ denotes the polar angle measured from the downstream jet axis (see figure 2) and the term in curly brackets should be interpreted as an operator that operates on everything to its right. So that, in particular, $\partial / \partial y_{i}$ acts on $e^{-i \omega y_{1} \cos \theta / c_{\infty}} \tilde{\boldsymbol{g}}_{v 4}^{a}$. This result is valid for all values of $\boldsymbol{y}_{\boldsymbol{T}}$, but 
when $y_{T}$ does not lie within the critical layer, equations(4.19) and (4.20) can be inserted to show that

$$
\begin{gathered}
\bar{\Gamma}_{i j}\left(\boldsymbol{x} \mid \boldsymbol{y}_{T}, Y\right) \equiv\left\{e^{i y_{1} \cos \theta \omega / c_{\infty}} \frac{\partial}{\partial y_{j}} \frac{\widetilde{c^{2} / \omega^{2}}}{\left[M\left(\boldsymbol{y}_{T}, Y\right) \cos \theta-1\right]^{2}} \frac{\partial}{\partial y_{i}} e^{-i \omega y_{1} \cos \theta / c_{\infty}}\right. \\
\left.-\frac{i \delta_{1 i}(\gamma-1)}{\omega} \frac{\partial U}{\partial y_{j}} \frac{1}{\left[M\left(\boldsymbol{y}_{T}, Y\right) \cos \theta-1\right]}\right\}\left(\varphi, \frac{\omega}{c_{\infty}} \cos \theta, \omega \mid \boldsymbol{y}_{T}, Y\right) \\
\bar{\Gamma}_{4 j}\left(\boldsymbol{x} \mid \boldsymbol{y}_{T}, Y\right)=\frac{i}{\omega} e^{i \omega y_{1} \cos \theta / c_{\infty}} \frac{\partial}{\partial y_{j}}\left[e^{-i \omega y_{1} \cos \theta / c_{\infty}} \frac{\widetilde{\mathcal{G}_{0}}\left(\varphi, \frac{\omega}{c_{\infty}} \cos \theta, \omega \mid \boldsymbol{y}_{T}, Y\right)}{M\left(\boldsymbol{y}_{T}, Y\right) \cos \theta-1}\right]
\end{gathered}
$$

where

$$
M\left(\boldsymbol{y}_{T}, Y\right) \equiv U\left(\boldsymbol{y}_{T}, Y\right) / c_{\infty}
$$

denotes the "acoustic" Mach number, $\widetilde{\mathcal{G}_{0}}\left(\varphi, k, \omega \mid \boldsymbol{y}_{T}, Y\right)$ is defined by

$$
\hat{G}_{0} \rightarrow{\frac{e}{\sqrt{x_{\perp}}}}^{-x_{\perp} \sqrt{k^{2}-\left(\omega / c_{\infty}\right)^{2}}} \widetilde{\mathcal{G}_{0}}\left(\varphi, k, \omega \mid \boldsymbol{y}_{\boldsymbol{T}}, Y\right), \quad \text { as } x_{\perp} \rightarrow \infty
$$

and the term in curly brackets should again be interpreted as an operator that operates on everything to its right.

This result is important because it provides an explicit representation of the singularity that will occur in the propagator when the parallel flow model is invoked. It shows, for example, that there is a singularity in the transverse source variable $\boldsymbol{y}_{T}$ whenever

$$
M\left(\boldsymbol{y}_{T}, Y\right)=1 / \cos \theta
$$

and that the strongest singularity in $\bar{\Gamma}_{i j}\left(\boldsymbol{x} \mid \boldsymbol{y}_{T}, Y\right)$, which results from differentiating the first term in(5.20), is $O\left(y_{\perp}^{-3}\right)$. This is a strong non-integrable singularity that will cause the far field pressure to become infinite and must, therefore, be eliminated (by using the uniformly valid non-parallel flow components given in Appendix $\mathrm{C}$ ) before meaningful predictions can be made.

Streamwise correlation lengths seem to be very long in certain regions of the flow (J. E. Bridges, personal communication) and it is therefore necessary to 
account for retarded time variations in that direction. But the transverse variation can also be important at sufficiently high Mach numbers. We assume that the transverse correlation lengths are small compared to the cross stream dimension, say $D$, of the mean flow. Then $\bar{\Gamma}_{\mu l}^{*}\left(\boldsymbol{x} \mid \boldsymbol{y}_{\boldsymbol{T}}+\boldsymbol{\xi}_{T}, Y ; \omega\right)$, which scales with $D$ and the acoustic wave length $c_{\infty} / \omega$, can only vary significantly over the correlation volume when $\omega D / c_{\infty}$ is large. In which case $\bar{\Gamma}_{\mu l}^{*}\left(\boldsymbol{x} \mid \boldsymbol{y}_{T}+\boldsymbol{\xi}_{T}, Y ; \omega\right)$ can be represented by its high frequency or WKBJ approximation* (Khavaran, Bridges \& Georgiadis, 2005)

$$
\bar{\Gamma}_{\mu l}^{*}\left(\boldsymbol{x} \mid \boldsymbol{y}_{T}, Y ; \omega\right) \approx A_{\mu l}\left(\boldsymbol{x} \mid \boldsymbol{y}_{\boldsymbol{T}}, Y ; \omega\right) \exp \left[i \frac{\omega}{c_{\infty}} S\left(\varphi, \theta \mid \boldsymbol{y}_{T}, Y\right)\right]
$$

where $A_{\mu l}\left(\boldsymbol{x} \mid \boldsymbol{y}_{T}, Y ; \omega\right)$ expands like

$$
A_{\mu l}^{(0)}\left(\boldsymbol{x} \mid \boldsymbol{y}_{\boldsymbol{T}}, Y\right)+\frac{\omega}{c_{\infty}} A_{\mu l}^{(1)}\left(\boldsymbol{x} \mid \boldsymbol{y}_{\boldsymbol{T}}, Y\right)+\left(\frac{\omega}{c_{\infty}}\right)^{2} A_{\mu l}^{(2)}\left(\boldsymbol{x} \mid \boldsymbol{y}_{\boldsymbol{T}}, Y\right) \cdots
$$

and $S\left(\varphi, \theta \mid \boldsymbol{y}_{T}, Y\right)$, which satisfies the Eikonal equation

$$
\left(\nabla_{\boldsymbol{y}_{\boldsymbol{T}}} S\right) \cdot\left(\nabla_{\boldsymbol{y}_{\boldsymbol{T}}} S\right)=\left[1-M\left(\boldsymbol{y}_{T}\right) \cos \theta\right]^{2} c_{\infty}^{2} / \widetilde{c^{2}}-\cos ^{2} \theta
$$

varies on the scale of $D$. It is, therefore, relatively constant over the correlation volume and can be expanded in a Taylor series for variations on this scale to obtain

$$
\bar{\Gamma}_{\mu l}^{*}\left(\boldsymbol{x} \mid \boldsymbol{y}_{\boldsymbol{T}}+\boldsymbol{\xi}_{T}, Y ; \omega\right) \approx \bar{\Gamma}_{\mu l}^{*}\left(\boldsymbol{x} \mid \boldsymbol{y}_{\boldsymbol{T}}, Y ; \omega\right) \exp \left[i \frac{\omega}{c_{\infty}} \boldsymbol{\xi}_{T} \cdot \nabla_{\boldsymbol{y}_{\boldsymbol{T}}} S\left(\varphi, \theta \mid \boldsymbol{y}_{\boldsymbol{T}}, Y\right)\right]
$$

which allows us to write the formula (5.14) for the far field spectrum in the purely algebraic form

$$
I_{\omega}(\boldsymbol{x} \mid \boldsymbol{y}) \rightarrow
$$

*Actually, the solution can be a sum of terms of this form, but the final result turns out to be the same and we therefore consider only the single term for the sake of simplicity. 


$$
\left(\frac{2 \pi}{x}\right)^{2} \frac{2 \pi \omega}{c_{\infty}} \sin \theta \bar{\Gamma}_{v j}\left(\boldsymbol{x} \mid \boldsymbol{y}_{T}\right) \bar{\Gamma}_{\mu l}^{*}\left(\boldsymbol{x} \mid \boldsymbol{y}_{T}\right) \Phi^{*}{ }_{v j \mu l}\left(\boldsymbol{y} ; \frac{\omega}{c_{\infty}} \cos \theta, \frac{\omega}{c_{\infty}} \nabla_{\mathrm{y}} S, \omega\left(1-M_{c} \cos \theta\right)\right)
$$

$$
\text { as } x \rightarrow \infty
$$

which depends on the turbulent source correlations only through the spectral tensor

$$
\Phi_{v j \mu l}^{*}\left(\boldsymbol{y} ; k_{1}, \boldsymbol{k}_{\perp}, \omega\right) \equiv \frac{1}{2 \pi} \int_{-\infty}^{\infty} e^{-i \omega \tau} \int_{V} e^{i\left(k_{1} \xi_{1}+\boldsymbol{k}_{T} \cdot \boldsymbol{\xi}_{\boldsymbol{T}}\right)} \boldsymbol{乛}_{v j \mu l}^{M}(\boldsymbol{y}, \boldsymbol{\xi}, \tau) d \boldsymbol{\xi} d \tau,
$$

where the asterisk is being used to denote complex conjugates. The comment following equation (5.23) implies that the strongest critical layer singularity in this result is now $O\left(y_{\perp}^{-6}\right)$ when non-parallel flow effects are neglected!

The presence of the enthalpy source $e_{4 j}^{\prime}$, significantly increases the complexity of these results. But equations (2.13),(2.14) and (2.23) suggest that the dimensionless ratio, $e_{4 j}^{\prime} / U_{J} e_{i j}^{\prime}$ (which determines the relative importance of $e_{4 j}^{\prime}$ and $e_{i j}^{\prime}$ in the acoustic analogy equations, where $U_{J}$ denotes a characteristic jet velocity)should be $O\left(v_{1}^{\prime} / U_{J}\right)$ for cold, i.e. unheated, jets when the Mach number is $O(1)$, (see, Lilley,1996 and Morfey, Szewczyk, \& Tester,1978), because $c^{2}$ is expected to be $O\left(v_{1}^{\prime}\right)^{2}$ in this case. The enthalpy source $e_{4 j}^{\prime}$ should then be small and can, therefore, be set to zero if we focus on cold jets-which we now do for the reminder of the paper. It is, of course, possible that equation (5.28) can still produce reasonable predictions even for hot jets. But this would require modeling many cross-coupling terms that would be difficult to estimate from any standard RANS based code. It may, however, be possible to do this by extending a RANS code to yield information about the RMS temperature fluctuations (as was done by Khavaran and Kenzakowski, 2007) if the velocity and sound speed fluctuations are assumed to be uncorrelated in the sense that

$$
\Phi^{*}{ }_{v j \mu l}\left(\boldsymbol{y} ; \frac{\omega}{c_{\infty}} \cos \theta, \frac{\omega}{c_{\infty}} \nabla_{\boldsymbol{y}_{T}} S, \omega\left(1-M_{c} \cos \theta\right)\right)=0
$$

when $\mu \neq v$ and either $\mu$ or $v=4$--but the result may be worse than neglecting $e_{4 j}^{\prime}$ entirely, because the velocity and enthalpy (sound speed) fluctuations may interfere destructively (Freund,2002).We, therefore, assume that $e_{4 j}^{\prime}=0$, i.e., that (5.30) holds whenever $\mu$ or $v$ is equal to 4. Then since (5.12) and (5.13) show 
that $\Phi^{*}{ }_{i j k l}$ is symmetric in its first two and last two indices, equation (5.28) can now be written as

$$
\begin{gathered}
I_{\omega}(\boldsymbol{x} \mid \boldsymbol{y}) \rightarrow \\
\left(\frac{2 \pi}{x}\right)^{2} \frac{2 \pi \omega}{c_{\infty}} \sin \theta G_{i j}\left(\boldsymbol{x} \mid \boldsymbol{y}_{T}\right) G_{k l}^{*}\left(\boldsymbol{x} \mid \boldsymbol{y}_{T}\right) \Phi_{i j k l}^{*}\left(\boldsymbol{y} ; \frac{\omega}{c_{\infty}} \cos \theta, \frac{\omega}{c_{\infty}} \nabla_{\mathrm{y}} S, \omega\left(1-M_{c} \cos \theta\right)\right) \\
\text { as } x \rightarrow \infty
\end{gathered}
$$

where the symmetric propagator

$$
G_{i j}\left(\boldsymbol{x} \mid \boldsymbol{y}_{T}\right)=G_{j i}\left(\boldsymbol{x} \mid \boldsymbol{y}_{T}\right) \equiv \frac{1}{2}\left[\bar{\Gamma}_{i j}\left(\boldsymbol{x} \mid \boldsymbol{y}_{T}\right)+\bar{\Gamma}_{j i}\left(\boldsymbol{x} \mid \boldsymbol{y}_{\boldsymbol{T}}\right)\right]
$$

still has six independent components, but only certain combinations of these appear in the simplified formulas (see section 6.1, below) used in the actual jet nose predictions presented in section 8 of this paper. Appendix $C$ uses the socalled "product rule" (Van Dyke,1975) along with the results of Section 4 to obtain appropriate uniformly valid expressions for these quantities.

The critical layer lies at the transverse location $\boldsymbol{y}_{T}=\boldsymbol{y}_{c}$ determined by(5.23). Figure 3 is a sketch of its overall configuration for a round jet. It shows that the critical layer usually moves inboard from the lip line toward the jet axis with increasing downstream distance and fixed $\theta$ and that, while the initial migration tends to be relatively slow, it quickly moves onto the jet centerline and disappears just after reaching the end of the potential core. The right angle intersection is shown because $U=U\left(y_{T}, Y\right)$ and

$$
U_{c}^{\prime} \equiv \partial U /\left.\partial y_{\perp}\right|_{y_{\perp}=y_{c}}=0 \text { for } y_{c}=0
$$

in this case while the data seems to suggest that $(\partial U / \partial Y)_{c}=O(1)$. So it follows from equation (B.2) that $d y_{c} / d Y$ must be infinite there. This behavior is expected to be generic because the on-axis mean-flow will usually behave as if it were axysymmetric.

The upstream critical layer thickness, say $\delta_{c}$, is also shown in figure 3. Equations (4.43) and (4.52) imply that it will become infinite when the critical layer reaches the jet axis, which would cause the critical layer solution (4.46) (4.51) to completely cancel out the outer solution singularity there. But equation (4.32) shows that the large value of $d y_{c} / d Y$ causes the critical layer expansion (4.28) to break down before this can occur and a new asymptotic solution will apply in the so called blending region indicated by the circle in figure 3 . But 
actually constructing this solution would be very difficult because the relevant flow structure (including that of the mean flow) is no longer quasi-parallel.

The weakly non-parallel flow structure appears to reemerge further downstream. But the outer parallel flow solution will remain invalid in the vicinity of the jet axis as long as the difference, say $\Delta U$, between the centerline velocity and the critical layer velocity $U_{\text {cr lay }} \equiv c_{\infty} / \cos \theta$ remains of order of the jet spread rate $\varepsilon$. We refer to this inner region as the modulated layer. The asymptotic structure and scaling of the relevant Green's function solution will differ from the critical layer structure in this region, but we do not derive the result here. The detailed analysis is similar to, but much more tedious than, that of section 4 . The primary difference is that the inner solution is now determined by a second order partial differential equation rather than by the ordinary differential equation(4.42). Fortunately, this equation can still be solved in closed form, but the solution is considerably more complex than the previous result. We can, however, simplify it by noticing that the streamwise length of this downstream region is typically much shorter than the streamwise length scale of the mean flow and that the RANS solutions show that the mean flow stagnation enthalpy $H_{0}=c_{p} \tilde{T}+U^{2} / 2$ is very nearly constant. The end result is that $\bar{\gamma}$ turns out to be proportional to

$$
\gamma_{0} \equiv \varsigma^{\left(1-\chi_{0}\right)} e^{\varsigma} \Gamma\left(\chi_{o}-1, \varsigma\right)
$$

where $\Gamma\left(\chi_{o}-1, \varsigma\right)$ denotes the incomplete Gamma function with argument $\varsigma$ given by

$$
\varsigma \equiv \frac{i k_{0} U^{\prime \prime}{ }_{c} \bar{y}_{\perp}^{2}}{4 V_{c}^{\prime}}, \quad \text { and } k_{0} \equiv \frac{\omega \cos \theta}{c_{\infty}}
$$

and scaled modulation parameter

$$
\chi_{0} \equiv \frac{-i k_{0}\left(\overline{\Delta U}+U_{c}^{(1)}\right)}{2 V_{c}^{\prime}}
$$

where

$$
\overline{\Delta U} \equiv\left(U_{c}-U_{\text {cr. lay. }}\right) / \varepsilon
$$

The appropriate lowest-order expressions for the relevant propagator components can be obtained by making the substitutions

$$
z_{0} \rightarrow \varsigma-\chi_{0}
$$




$$
w^{\prime}\left(z_{0}\right) \rightarrow \frac{1}{2}\left\{\frac{\partial}{\partial \varsigma}+\frac{(\gamma-1) U_{c}^{2}}{2 V_{c}^{\prime} \widetilde{c}_{0}^{2}} \frac{\partial U_{c}}{\partial Y} \frac{1}{\varsigma}\left[\left(1-\chi_{0}\right) \frac{\partial}{\partial \chi_{0}}-\frac{\partial}{\partial \varsigma} \varsigma\right]\right\} \gamma_{0}\left(\varsigma, \chi_{0}\right)
$$

in equations (6.27) (below) and (C.18) to (C.22), where $z_{0}$ is defined by (6.15) (below).This solution merges smoothly into the parallel flow solution in the downstream potion of this region where $\left|\chi_{0}\right|$ becomes large. But it must also merge onto the blending layer solution at the upstream part of the region. We do not attempt to construct this solution here -which cannot, in any case be done analytically. But since the thickness of the upstream critical layer is infinite when it becomes perpendicular to the streamwise axis, we expect the blending-layer solution to also spread out into the axial direction and smoothly merge into the downstream region also shown in figure 3.

\section{Source Modeling}

The previous sections were primarily concerned with the purely acoustic effects, frequently - but somewhat misleadingly-- referred to as propagation effects. The remaining ingredient involves the (near field) fluid mechanical effects, which are inputted by modeling the turbulent sources that enter the acoustic formulas through the modified spectral

tensor $\Phi^{*}{ }_{i j k l}\left(\boldsymbol{y} ; \frac{\omega}{c_{\infty}} \cos \theta, \frac{\omega}{c_{\infty}} \nabla_{\boldsymbol{y}_{T}} S, \omega\left(1-M_{c} \cos \theta\right)\right)$.

Ideally, we would like to model $\Phi_{i j k l}$ directly, but the models must be based on experimental data and the experimentalists are unlikely to provide the requisite data in the near future. A possible alternative is to develop models for the ordinary spectral tensor

$$
\Psi_{i j k l}\left(\boldsymbol{y} ; \boldsymbol{k}_{1}, \boldsymbol{k}_{\perp}, \omega\right) \equiv \frac{1}{2 \pi} \int_{-\infty}^{\infty} e^{-i \omega \tau} \int_{V} e^{i\left(k_{1} \xi_{1}+\boldsymbol{k}_{T} \cdot \boldsymbol{\xi}_{T}\right)} R_{i j k l}^{M}(\boldsymbol{y}, \boldsymbol{\xi}, \tau) d \boldsymbol{\xi} d \tau,
$$

which is implicitly related to $\Phi^{*}{ }_{i j k l}$ by (5.13). But even this quantity has almost never been measured (see, for example, Harper-Bourne (2003))--and then only at very low Mach numbers. Various components of the two point correlation tensor appear to be the most extensively measured turbulence quantities in high speed air jets. We are, therefore, forced to develop models for these quantities and calculate the spectra from(6.1).

There are two main requirements for such models. The first is that the large number of independent components (there are 45 of these in all) be brought down to a manageable level and the second is that realistic, but still relatively simple models, can be obtained for those components. The first of these (which is discussed in the next section) is usually accomplished by introducing add hoc 
symmetry and/or statistical assumptions such as local isotropy and quasinormality. But, as indicated in the introduction, such assumptions are not viable at the high Mach numbers of technological interest. A more realistic approach is therefore introduced in the next section. Specific models for the remaining spectral components are introduced in section 6.2.

\subsection{Kinematic Modeling}

As indicated in the introduction, the usual quasi-normal and local isotropy assumptions are untenable at high Mach numbers, but developing a viable formulation for a general non-normal axisymmetric source model (Batchelor,1953; Goldstein, and Rosenbaum,1973 b, Kerschen,1983) is enormously complex and the result would probably be too complicated to be of practical utility. We therefore proceed by developing a non-normal but isotropic model and an axisymmetric but quasinormal model.

6.1a General (non quasinormal) Isotropic Turbulence

Equation(5.13) shows that $\mathcal{R}_{i j k l}^{M}$ will be an isotropic tensor if $R_{i j k l}^{M}$ is an isotropic tensor. Batchelor (1953) points out that the most general fourth order isotropic tensor is of the form

$$
\begin{aligned}
\mathbb{R}_{i j k l}^{M}=A \xi_{i} \xi_{j} \xi_{k} \xi_{l}+B \xi_{i} \xi_{j} \delta_{k l}+C \xi_{i} \xi_{k} \delta_{j l} & +D \xi_{i} \xi_{l} \delta_{j k}+E \xi_{j} \xi_{k} \delta_{i l}+F \xi_{j} \xi_{l} \delta_{i k} \\
& +G \xi_{k} \xi_{l} \delta_{i j}+H \delta_{i j} \delta_{k l}+I \delta_{i k} \delta_{j l}+J \delta_{i l} \delta_{j k}
\end{aligned}
$$

where $A, B, \ldots J$ are functions of $\boldsymbol{y}, t$ and $|\boldsymbol{\xi}|$. But it follows from the definition of $\boldsymbol{R}_{i j k l}^{M}$ that

and, therefore, that

$$
\boldsymbol{R}_{i j k l}^{M}=\boldsymbol{R}_{j k l}^{M}=\boldsymbol{R}_{i j k k}^{M}
$$

$$
\begin{aligned}
\mathbb{R}_{i j k l}^{M}=A \xi_{i} \xi_{j} \xi_{k} \xi_{l} & +B \xi_{i} \xi_{j} \delta_{k l}++G \xi_{k} \xi_{l} \delta_{i j}+C \xi_{i}\left(\xi_{k} \delta_{j l}+\xi_{l} \delta_{j k}\right) \\
& +E \xi_{j}\left(\xi_{k} \delta_{i l}+\xi_{l} \delta_{i k}\right)+H \delta_{i j} \delta_{k l}+I\left(\delta_{i k} \delta_{j l}+\delta_{i l} \delta_{j k}\right)
\end{aligned}
$$

which can be integrated over the unit sphere to show that

$$
\int_{V} \boldsymbol{R}_{i j k l}^{M}\left(\boldsymbol{y} ; \boldsymbol{\xi}, t^{\prime \prime}\right) d \boldsymbol{\xi}=\mathbb{I}_{1} \delta_{i j} \delta_{k l}+\mathbb{I}_{2}\left(\delta_{i k} \delta_{j l}+\delta_{i l} \delta_{j k}\right)
$$

where 


$$
\begin{aligned}
\mathbb{I}_{1}\left(\boldsymbol{y} ; t^{\prime \prime}\right) \equiv 4 \pi \int_{0}^{\infty}\left[\frac{1}{15} A \xi^{4}+\frac{1}{3}(B+G) \xi^{2}+H\right] \xi^{2} d \xi \\
\quad=\int_{V} \boldsymbol{R}_{1111}^{M}\left(\boldsymbol{y} ; \boldsymbol{\xi}, t^{\prime \prime}\right) d \boldsymbol{\xi}-2 \int_{V} \boldsymbol{R}_{1212}^{M}\left(\boldsymbol{y} ; \boldsymbol{\xi}, t^{\prime \prime}\right) d \boldsymbol{\xi}
\end{aligned}
$$

and

$$
\mathbb{I}_{2}\left(y ; t^{\prime \prime}\right) \equiv 4 \pi \int_{0}^{\infty}\left[\frac{1}{15} A \xi^{4}+\frac{1}{3}(C+E) \xi^{2}+I\right] \xi^{2} d \xi=\int_{V} \boldsymbol{R}_{1212}^{M}\left(y ; \xi, t^{\prime \prime}\right) d \xi
$$

Inserting this into equation (5.29) shows that

$$
\Phi_{i j k l}^{*}(\boldsymbol{y} ; 0,0, \omega)=\tilde{\phi}_{1} \delta_{i j} \delta_{k l}+\tilde{\phi}_{2}\left(\delta_{i k} \delta_{j l}+\delta_{i l} \delta_{j k}\right)
$$

where

$$
\begin{gathered}
\tilde{\phi}_{1} \equiv \Phi_{1111}^{*}(\boldsymbol{y} ; 0, \boldsymbol{0}, \omega)-2 \Phi_{1212}^{*}(\boldsymbol{y} ; 0,0, \omega) \\
\tilde{\phi}_{2} \equiv \Phi^{*}{ }_{1212}(\boldsymbol{y} ; 0,0, \omega)
\end{gathered}
$$

with

$$
\Phi_{1212}^{*}(y ; 0,0, \omega)=\Phi_{1313}^{*}(y ; 0,0, \omega)
$$

It is reasonable to assume that $\Phi_{i j k l}^{*}\left(\boldsymbol{y} ; k_{1}, \boldsymbol{k}_{T}, \omega\right)$ has the same symmetry, i.e. that

$$
\Phi_{i j k l}^{*}\left(\boldsymbol{y} ; k_{1}, \boldsymbol{k}_{T}, \omega\right)=\phi_{1} \delta_{i j} \delta_{k l}+\phi_{2}\left(\delta_{i k} \delta_{j l}+\delta_{i l} \delta_{j k}\right)
$$

where

$$
\begin{aligned}
& \phi_{1} \equiv \Phi_{1111}^{*}\left(\boldsymbol{y} ; k_{1}, \boldsymbol{k}_{T}, \omega\right)-2 \Phi_{1212}^{*}\left(\boldsymbol{y} ; k_{1}, \boldsymbol{k}_{T}, \omega\right) \\
& \phi_{2} \equiv \Phi_{1212}^{*}\left(\boldsymbol{y} ; k_{1}, \boldsymbol{k}_{T}, \omega\right)
\end{aligned}
$$

and

$$
\Phi_{1212}^{*}\left(\boldsymbol{y} ; k_{1}, \boldsymbol{k}_{T}, \omega\right)=\Phi_{1313}^{*}\left(\boldsymbol{y} ; k_{1}, \boldsymbol{k}_{T}, \omega\right)
$$


Inserting these results into equation(5.31) and using Appendix D (which contains the relevant details of the analysis) as well as (C.18) to (C.22) yields

$$
\begin{gathered}
I_{\omega}(\boldsymbol{x} \mid \boldsymbol{y}) \rightarrow\left(\frac{2 \pi}{x}\right)^{2} \frac{2 \pi \omega}{c_{\infty}} \sin \theta \|\left[\Phi^{*}{ }_{1111}+4 \operatorname{Re}_{c}\left(C_{c}^{*}-1\right) \Phi^{*}{ }_{1212}\right]\left|\widetilde{\mathcal{G}_{0}}\left[1+w^{\prime}\left(z_{0}\right)\right]\right|^{2} \\
+4\left\{\frac{1}{\omega^{2}}\left|\frac{\sqrt{c^{2}} \cos \theta}{c_{\infty}(M \cos \theta-1)} \nabla_{T}\left[\frac{\widetilde{\mathcal{G}_{0}} \sqrt{\widetilde{c^{2}}}}{(1-M \cos \theta)}\right]-\frac{(\gamma-1) \widetilde{\mathcal{G}_{0}} c_{\infty}}{2(1-M \cos \theta)} \nabla_{T} M\right|^{2}\right. \\
\left.+\left(\left|G_{23}\right|^{2}-\operatorname{Re}_{22} \Gamma_{33}^{*}\right)\right\}\left|z_{0}^{3} w^{\prime \prime}\left(z_{0}\right)\right|^{2} \Phi^{*}{ }_{1212} \|
\end{gathered}
$$

where the primes denote differentiation with respect to

$$
z_{0} \equiv \alpha_{0} y_{\perp},
$$

$\alpha_{0}^{2}$ denotes the parameter $\alpha^{2}$ (defined by (4.43)) with $k=\omega \cos \theta / c_{\infty}, C_{c}$ is given by

$$
C_{c} \equiv \frac{-C^{2} 2 z_{0}^{2} w^{\prime}\left(z_{0}\right)}{1+w^{\prime}\left(z_{0}\right)}=\frac{-\widetilde{c^{2}} 2 z_{0}^{2} w^{\prime}\left(z_{0}\right) \cos ^{2} \theta}{c_{\infty}^{2}\left[M\left(\boldsymbol{y}_{T}\right) \cos \theta-1\right]^{2}\left[1+w^{\prime}\left(z_{0}\right)\right]}
$$

(with $C$ defined by (C.4) and the replacements (5.38) and (5.39) for $z_{0}$ and, respectively inserted after the critical layer disappears) and

$$
\begin{gathered}
\Phi^{*}{ }_{1111} \equiv \Phi^{*}{ }_{1111}\left(\boldsymbol{y} ; \frac{\omega}{c_{\infty}} \cos \theta, \frac{\omega}{c_{\infty}} \nabla_{\boldsymbol{y}_{T}} S, \omega\left(1-M_{c} \cos \theta\right)\right), \\
\Phi^{*}{ }_{1212} \equiv \Phi^{*}{ }_{1212}\left(\boldsymbol{y} ; \frac{\omega}{c_{\infty}} \cos \theta, \frac{\omega}{c_{\infty}} \nabla_{\boldsymbol{y}_{T}} S, \omega\left(1-M_{c} \cos \theta\right)\right)
\end{gathered}
$$

are two independent components of the Doppler shifted turbulence spectrum that can, in principle, have entirely different functional forms.

\section{1b Quasinormal Axisymmetric Model}


We now put

$$
R_{i j}^{M}(\boldsymbol{y}, \boldsymbol{\xi}, \tau) \equiv \frac{1}{2 T} \int_{-T}^{T} \sqrt{\rho} v_{i}^{\prime}(\boldsymbol{y}, t) \sqrt{\rho} v_{j}^{\prime}\left(\boldsymbol{y}+\boldsymbol{\xi}+\hat{\boldsymbol{i}} U_{c} \tau, t+\tau\right) d t
$$

and assume that the turbulence is quasi-normal(see Batchelor,1953; Goldstein,2004), which means that

$$
R_{i j k l}^{M}(\boldsymbol{y}, \boldsymbol{\xi}, \tau)=R_{i k}^{M}(\boldsymbol{y} ; \boldsymbol{\xi}, \tau) R_{j l}^{M}(\boldsymbol{y} ; \boldsymbol{\xi}, \tau)+R_{i l}^{M}(\boldsymbol{y} ; \boldsymbol{\xi}, \tau) R_{j k}^{M}(\boldsymbol{y} ; \boldsymbol{\xi}, \tau)
$$

since (Goldstein \& Leib,2005)

$$
\begin{aligned}
\frac{1}{2 T} \int_{-T}^{T} \rho v_{i}^{\prime} v_{j}^{\prime}\left(\boldsymbol{y}, \tau_{0}\right) \rho v_{k}^{\prime} v_{l}^{\prime}\left(\boldsymbol{y}+\boldsymbol{\eta}, \tau_{0}+\tau\right) d \tau_{o} \\
=R_{i j k l}(\mathbf{y}, \boldsymbol{\eta}, \tau)+R_{i j}(\mathbf{y} ; \mathbf{0}, 0) R_{k l}(\mathbf{y}+\boldsymbol{\eta} ; \mathbf{0}, 0)
\end{aligned}
$$

We now require the turbulence to be axisymmetric (Batchelor, 1953) in the sense that

$$
R_{i j}^{M}(\boldsymbol{y} ; \boldsymbol{\xi}, \tau)=A \xi_{i} \xi_{j}+B \delta_{i j}+C \delta_{1 i} \delta_{1 j}+D\left(\delta_{1 i} \xi_{j}+\delta_{1 j} \xi_{i}\right)
$$

where $A, B, C$, and $D$ denote functions of $\boldsymbol{y}, \quad \tau, \quad \xi_{\perp} \equiv \sqrt{\xi_{2}^{2}+\xi_{3}^{2}}$ and $\xi_{1}$; $A, B$ and $C$ are even functions of the latter quantity while $D$ is an odd function. This model is chosen because it is the most general of those whose mathematical properties have been studied in the literature and because it reflects the fact that the cross flow velocity components tend to be much more similar to one another than to the stream-wise component (even for nonaxisymmetric flows).

It follows from equations(5.13), (6.19) and (6.21) that

$$
\begin{aligned}
\frac{1}{2} G_{i j} G_{k l}^{*} \int_{V} \boldsymbol{R}_{i j k l}^{M}(\boldsymbol{y} ; \boldsymbol{\xi}, \tau) d \xi=G_{i j} G_{k l}^{*} I_{i k j l}-2\left(\frac{\gamma-1}{2}\right) & \operatorname{Re}_{i i} G_{k l}^{*} I_{n k n l} \\
& +\left(\frac{\gamma-1}{2}\right)^{2}\left|G_{i i}\right|^{2} I_{n k n k}
\end{aligned}
$$


where

$$
I_{i j k l} \equiv \int_{V} R_{i j}^{M} R_{k l}^{M} d \xi
$$

while appendix $\mathrm{E}$ (which contains the relevant details for this model) and equations(C.18) to (C.22) imply that

$$
\begin{aligned}
& G_{i j} G_{k l}^{*} I_{i k j l} \\
& =\left[\Phi^{*}{ }_{1111}\left|C_{c}\right|^{2}+2 \Phi^{*}{ }_{1122} \operatorname{Re}\left(C_{c}\left(1-C_{c}^{*}\right)\right)+\left|1-C_{c}\right|^{2} \Phi^{*}{ }_{2222}\right] \mid \widetilde{\mathcal{G}_{0}}\left[1+\left.w^{\prime}\left(z_{0}\right)\right|^{2}\right. \\
& +2 \operatorname{Re}\left(G_{22} G_{33}^{*}-\left|G_{23}\right|^{2}\right)\left(I_{2323}-I_{2222}\right)\left|z_{0}^{3} w^{\prime \prime}\left(z_{0}\right)\right|^{2}+ \\
& \frac{2}{\omega^{2}}\left|\frac{\sqrt{c^{2}} \cos \theta}{c_{\infty}(M \cos \theta-1)} \nabla_{T}\left[\frac{\widetilde{\mathcal{G}_{0}} \sqrt{\widetilde{c^{2}}}}{(1-M \cos \theta)}\right]-\frac{(\gamma-1) \widetilde{\mathcal{G}_{0}} c_{\infty}}{2(1-M \cos \theta)} \nabla_{T} M\right|\left|z_{0}^{3} w^{\prime \prime}\left(z_{0}\right)\right|^{2}\left(I_{1122}+I_{1212}\right)
\end{aligned}
$$

where $C_{c}$ is defined by equation(6.16). As in the preceding sub-section, we now assume that this result along with the symmetry conditions

$$
\Phi_{2222}^{*}=\Phi_{3333}^{*}, \quad \Phi_{1122}^{*}=\Phi_{1133}^{*}, \quad \Phi_{1212}^{*}=\Phi_{1313}^{*}
$$

also holds when $\int_{V} \boldsymbol{乛}_{i j k l}^{M}(\boldsymbol{y} ; \boldsymbol{\xi}, \tau) d \boldsymbol{\xi}$ is replaced by

$$
\int_{V} \exp i \frac{\omega}{c_{\infty}}\left(\xi_{1} \cos \theta+\xi_{T} \cdot \nabla_{\mathbf{y}_{\mathbf{T}}} S\right) \boldsymbol{R}_{i j k l}^{M}(\boldsymbol{y} ; \boldsymbol{\xi}, \tau) d \boldsymbol{\xi} .
$$

Then, inserting (6.24),along with equations(6.19),(C.18),(E.3) and (E.4) into (6.22), and using the result in equations (5.31), (5.29) and (5.13), shows that the far-field acoustic spectrum $I_{\omega}(\boldsymbol{x} \mid \boldsymbol{y})$ can now be expressed in terms of five distinct components of the spectral tensor

$$
\Phi_{i j k l}^{*} \equiv \Phi^{*}{ }_{i j k l}\left(\boldsymbol{y} ; \frac{\omega}{c_{\infty}} \cos \theta, \frac{\omega}{c_{\infty}} \nabla_{\boldsymbol{y}_{T}} S, \omega\left(1-M_{c} \cos \theta\right)\right)
$$


by

$$
\begin{aligned}
& I_{\omega}(\boldsymbol{x} \mid \boldsymbol{y}) \rightarrow \\
& \left(\frac{2 \pi}{x}\right)^{2} \frac{2 \pi \omega}{c_{\infty}} \sin \theta\left\{\left[\Phi{ }^{*}{ }_{1111}\left|C_{c}\right|^{2}+2 \Phi^{*}{ }_{1122} \operatorname{Re}\left(C_{c}\left(1-C_{c}^{*}\right)\right)+\Phi^{*}{ }_{2222}\left|1-C_{c}\right|^{2}\right]\left|\widetilde{\mathcal{G}_{0}}\left[1+w^{\prime}\left(z_{0}\right)\right]\right|^{2}\right. \\
& \left.+4 \mid \frac{\sqrt{c^{2}} \cos \theta}{c_{\infty}(M \cos \theta-1)} \nabla_{T}\left(\frac{\widetilde{\mathcal{G}_{0}} \sqrt{\widetilde{c}^{2}}}{\omega(1-M \cos \theta)}\right)-\frac{(\gamma-1) \widetilde{\mathcal{G}_{0}} c_{\infty}}{2 \omega(1-M \cos \theta)} \nabla_{T} M\right]\left.z_{0}^{3} w^{\prime \prime}\left(z_{0}\right)\right|^{2} \Phi_{1212}^{*} \\
& \left.+2\left(\left|G_{23}\right|^{2}-\operatorname{Re}_{22} \Gamma_{33}^{*}\right)\left|z_{0}^{3} w^{\prime \prime}\left(z_{0}\right)\right|^{2}\left(\Phi^{*}{ }_{2222}-\Phi^{*}{ }_{2233}\right)\right\}
\end{aligned}
$$

with the replacements (5.38) and (5.39) being made just downstream of the critical layer. And since

$$
\Phi *_{1111}=\Phi^{*}{ }_{2222}=2 \Phi^{*}{ }_{1212}+\Phi^{*}{ }_{1122} \text { and } \quad \Phi^{* 2233}=\Phi_{1122}^{*}
$$

for isotropic turbulence, this result reduces to equation(6.14) in that case, even though the latter applies when the turbulence is not quasi-normal. This shows that the quasi-normal hypothesis does not effect the isotropic limit of equation (6.27). An alternate representation of the source terms was given by Musifar $(1993,2006)$

Equation (6.27) can be rewritten by adding $4 \mathbb{R e}_{e}\left[C_{c}\left(1-C_{c}^{*}\right)\right] \Phi^{*}{ }_{1212}$ to the first term in square brackets and subtracting the corresponding result from the second. The modified first term will then be proportional to $\Phi{ }_{1111}\left|C_{c}\right|^{2}+2 \operatorname{Re}\left[C_{c}\left(1-C_{c}^{*}\right)\right]\left(2 \Phi{ }_{1212}+\Phi^{*}{ }_{1122}\right)+\left|1-C_{c}\right|^{2} \Phi^{*}{ }_{2222}$, which reduces to $\Phi^{*}{ }_{1111}$ when the turbulence is isotropic, and in the more general non-isotropic case (see Equation(6.16)), becomes identical to the self noise term found by Goldstein \& Rosenbaum (1973 b) in the Lighthill equation context when the acoustic Mach number $M$ is set equal to zero (see Ribner (1969)). The remaining (modified) terms can be shown to vanish in the absence of mean shear (i.e., 
when $M \equiv 0, \widetilde{c^{2}}=c_{\infty}^{2}$ and $\widetilde{\mathcal{G}_{0}}$ is determined from the free space Green's function). The vanishing of the last term is a direct consequence of replacing $\widetilde{\mathcal{G}_{0}}$ by the free space Green's function while the second term vanishes because the subtracted $\operatorname{Re}\left[C_{c}\left(1-C_{c}^{*}\right)\right]\left|\widetilde{\mathcal{G}_{0}}\right|^{2}$ term cancels with $\left|\nabla_{T}\left(\widetilde{\mathcal{G}_{0}} / \omega\right)\right|^{2}$ in this case. It would, therefore, not be unreasonable to follow Khavaran \& Bridges (2004) and refer to the remaining (modified) terms as the shear noise terms. Notice that they are both proportional to $\Phi^{*}{ }_{1212}$ when the turbulence is isotropic (but not necessarily quasi-normal, see(6.27)). This latter spectral component becomes proportional to $\Phi^{*}{ }_{1111}$ when the turbulence is also assumed to be quasi-normal, but $\Phi^{*}{ }_{1212}$ and $\Phi{ }^{*}{ }_{1111}$ can be independently specified in the more general case being considered here (which implies that the shear and self noise spectra can be independently specified). A similar decomposition was made by Musifar (1992).

The $1^{\text {st }}$ term in equation (6.27) can also be rewritten by collecting coefficients of $\left|C_{c}\right|^{2}, \operatorname{Re}_{c}$ and 1. It can be shown that it is the same as the result that would be obtained if the modified tensor $\boldsymbol{R}_{i j k l}^{M}$ were assumed to be quasi-normal with the corresponding $2^{\text {nd }}$ order tensors being axisymmetric.

Equation (6.27) contains five modified spectral tensor components. Equations(5.13), (5.29) and (6.23) imply that they can be expressed in terms of five distinct components of the spectrum (6.1) of the Reynolds Stress autocovariance tensor, which, with the present methodology, can be specified independently of one another. It follows from (6.16)and the far field behavior the Fourier transformed Green's function, that the coefficient of $\Phi^{*}{ }_{2222}$ and the second term in the absolute value squared multiplying $\Phi^{*}{ }_{1212}$ are the only two terms in (6.27) that do not have a $\cos \theta$ or a $\cos ^{2} \theta$ factor. The entire $90^{\circ}$ sound field is therefore produced by these terms. But our numerical computations indicate that the contribution from the latter term is, as expected, negligibly small.

As written equation (6.27) is the sum of three distinct terms: 1 ) the term containing the square bracket (which we refer to as term $I$ ), 2) the term involving $\Phi_{1212}^{*}$ (which we refer to as term $I I$ ), and 3) the remaining term(referred to as term $I I I$ ). The first of these only involves the 1111, the 2222. and the 1122 components of the Reynolds stress autocovariance tensor. The second only involves the 1212 component and the last the 2222 and the 2233 components. Terms $I$ and $I I I$, therefore only depend on the covariances of the squares of velocity components, whereas term II only involves the autocovariance of the cross velocity components. Notice that the first member of the latter term has 6 powers of the Doppler factor $1-M \cos \theta$ in its denominator when the implied differentiation is carried out - which will cause it to produce a very large contribution to the small angle sound field at supersonic Mach numbers. (The other terms in the equation only have at most 4 powers of the Doppler factor in their denominators.) 


\subsection{Spectral Modeling}

Experiments (Bridges \& Podboy, 1999; Harper-Bourne, 2003) suggest that $R_{i j k l}^{M}$ cannot be expressed as a simple product of its space and time components ( as was often assumed in the past, e.g., Goldstein and Rosenbaum,1973 b) but that a good local model, which is consistent with axi-symmetry but does not account for the potentially significant long range coherence effects that are most effected by the streamwise retarded time variations, might be

$$
R_{i j k l}^{M}=\mathrm{a}_{0,0} e^{-X+\tilde{\gamma}}
$$

where

$$
X\left(\tilde{\xi}_{1}, \tilde{\xi}_{T}, \tilde{\tau}\right) \equiv \sqrt{\tilde{\xi}_{1}^{2}+\beta^{2}+\tilde{\tau}^{2}}=\sqrt{\hat{\xi}_{1}^{2}+\beta^{2}} \quad \text { with } \quad \hat{\xi}_{1}^{2} \equiv \tilde{\xi}_{1}^{2}+\tilde{\tau}^{2}
$$

$\beta^{2}=\beta^{2}\left(\tilde{\xi}_{T}\right)$ and $\tilde{\gamma}^{2}=\tilde{\gamma}^{2}\left(\tilde{\xi}_{T}\right)$ are arbitrary functions of $\tilde{\xi}_{T}$ which vanish at

$\tilde{\xi}_{T}=0, \mathrm{a}_{0,0}$ is a parameter, the tildes denote the normalized variables

$\tilde{\xi}_{1} \equiv \xi_{1} / l_{1}, \tilde{\xi}_{T} \equiv \xi_{T} / l_{T}, \tilde{\tau} \equiv \tau \lambda$, with $\xi_{T} \equiv \sqrt{\xi_{2}^{2}+\xi_{3}^{2}}$ and the intermediate length and time scales $l_{1}, l_{\perp}$, and $1 / \lambda$ can be related to the integral scales measured by (Bridges \& Podboy, 1999).

Notice that $R_{i j k l}^{M}\left(\boldsymbol{y}, \boldsymbol{\xi}_{1}, \boldsymbol{0}, 0\right)$ and $R_{i j k l}^{M}(\boldsymbol{y}, 0, \boldsymbol{0}, \tau)$ exhibit the usual cusps at $\xi_{1}=0$ and $\tau=0$, respectively, that are observed in virtually all high Reynolds number experiments (Pope, 2000, pp. 67, 70, 71,110, figures 3.20, 3.22 (a), 5.13). Of course, these correlations must, in reality, be smooth functions of their arguments even at these points, but the corresponding radii of curvature turn out to be of the order of the Taylor microscale (Pope, 2000, pp.198-200) which would certainly be negligibly small at the high Reynolds numbers that are of interest here.

Since (5.13), (5.29) and the symmetry conditions (6.25) imply that $R_{2222}^{M}\left(\boldsymbol{y}, \boldsymbol{\xi}_{1}, \boldsymbol{\xi}_{T}, \tau\right)$ can be replaced by $\frac{1}{2}\left[R_{2222}^{M}\left(\boldsymbol{y}, \xi_{1}, \boldsymbol{\xi}_{T}, \tau\right)+R_{3333}^{M}\left(\boldsymbol{y}, \xi_{1}, \boldsymbol{\xi}_{T}, \tau\right)\right]$ in

$\Phi^{*}{ }_{2222}\left(\boldsymbol{y} ; \boldsymbol{k}_{1}, \boldsymbol{k}_{T}, \omega\left(1-M_{c} \cos \theta\right)\right)$, that $R_{1122}^{M}\left(\boldsymbol{y}, \boldsymbol{\xi}_{1}, \boldsymbol{\xi}_{T}, \tau\right)$ can be replaced by $\frac{1}{2}\left[R_{1122}^{M}\left(\boldsymbol{y}, \boldsymbol{\xi}_{1}, \boldsymbol{\xi}_{T}, \tau\right)+R_{1133}^{M}\left(\boldsymbol{y}, \boldsymbol{\xi}_{1}, \boldsymbol{\xi}_{T}, \tau\right)\right]$ in $\Phi^{*}{ }_{1122}\left(\boldsymbol{y} ; \boldsymbol{k}_{1}, \boldsymbol{k}_{T}, \omega\left(1-M_{c} \cos \theta\right)\right)$, etc, and since these latter quantities depend on $\xi_{T}$ only through $\xi_{T}=\sqrt{\xi_{2}^{2}+\xi_{3}^{2}}$, a reasonable global model that has the same local characteristics as (6.29) but is less compact (and, therefore, able to reproduce the negative loops in the correlation tensor (Bridges \& Podboy (1999)) would, then be 


$$
\begin{aligned}
R_{i j k l}^{M}=\sum_{m=0}^{\infty} \sum_{l=0}^{\infty} a_{m, l} D_{1}^{m} D_{\tau}^{l} e^{-X+\tilde{\gamma}}= & \\
& \left\{a_{0,0}-\frac{a_{0,1} \tilde{\tau}^{2}+a_{1,0} \tilde{\xi}_{1}^{2}}{X}+\frac{a_{1,1} \tilde{\tau}^{2} \tilde{\xi}_{1}^{2}}{X^{2}}\left(1+\frac{1}{X}\right)+\ldots\right\} e^{-X+\tilde{\gamma}},
\end{aligned}
$$

where the $a_{m, l}$ are parameters, the operators $D_{\kappa}^{n}, \kappa=\tau, 1$ which are defined by

$$
D_{1} \equiv \tilde{\xi}_{1} \frac{\partial}{\partial \tilde{\xi}_{1}}, \quad D_{\tau} \equiv \tilde{\tau} \frac{\partial}{\partial \tilde{\tau}}, D_{\kappa}^{0} \equiv 1, D_{\kappa}^{n} \equiv D_{\kappa} \ldots D_{\kappa}, n \text { times, }
$$

satisfy the commutation relations $D_{\kappa} D_{\lambda}=D_{\lambda} D_{\kappa}$.

Then since (5.12)implies that

$$
a_{0,0}=R_{i j k l}(\boldsymbol{y} ; \boldsymbol{0}, 0) \equiv \frac{1}{2 T} \int_{-T}^{T}\left[\rho v_{i}^{\prime} v_{j}^{\prime}-\overline{\rho v_{i}^{\prime} v_{j}^{\prime}}\right]\left(\boldsymbol{y}, \tau_{0}\right)\left[\rho v_{k}^{\prime} v_{l}^{\prime}-\overline{\rho v_{k}^{\prime} v_{l}^{\prime}}\right]\left(\boldsymbol{y}, \tau_{0}\right) d \tau_{0}
$$

must be different for the different components of $R_{i j k l}^{M}$ it is reasonable to expect that the remaining $a_{m, l}$ will be different as well.

The corresponding spectral tensor is

$$
\Psi_{i j k l}\left(k_{1}, \boldsymbol{k}_{T}, \omega\right)=\frac{l_{1} l_{T}^{2}}{\lambda} \iint_{-\infty}^{\infty} \int_{-\infty}^{\infty} e^{i\left(\xi_{1} k_{1}+\boldsymbol{\xi}_{T} \cdot \boldsymbol{k}_{T}-\omega \tau\right)} \sum_{m, l=0}^{\infty} a_{m, l} D_{1}^{m} D_{\tau}^{l} e^{-X+\gamma} d \tilde{\boldsymbol{\xi}}_{T} d \tilde{\tau} d \tilde{\xi}_{1}
$$

which, as shown in appendix $\mathrm{F}$ (which contains the details of the derivation), can be written as

$$
\Psi_{i j k l}\left(k_{1}, \boldsymbol{k}_{T}, \omega\right)=-\frac{2 \pi l_{1} l_{T}^{2}}{\lambda} \sum_{m, l=0}^{\infty} a_{m, l}(-1)^{m+l} D_{k_{1}}^{m} D_{\omega}^{l} \frac{1}{R} \frac{d}{d R} G_{0}\left(\tilde{k}_{T}, R\right)
$$

where the operators $D_{k_{1}}, D_{\omega}$ are defined by

$$
D_{k_{1}} \equiv \frac{\partial}{\partial k_{1}} k_{1}=\left(\frac{\partial}{\partial \tilde{k}_{1}} \tilde{k}_{1}\right)_{\tilde{k}_{T}, \tilde{\omega}=\text { const. }}, \quad D_{\omega} \equiv \frac{\partial}{\partial \omega} \omega=\left(\frac{\partial}{\partial \tilde{\omega}} \tilde{\omega}\right)_{\tilde{k}_{T}, \tilde{k}_{1}=\text { const. }}
$$


we have put $\tilde{k}_{1} \equiv k_{1} l_{1}, \quad \tilde{\omega} \equiv \omega / \lambda, \quad \tilde{\boldsymbol{k}}_{T} \equiv \boldsymbol{k}_{T} l_{T}$

$$
R \equiv \sqrt{\tilde{k}_{1}^{2}+\tilde{\omega}^{2}}
$$

and

$$
\begin{gathered}
G_{0}\left(\tilde{k}_{T}, R\right) \equiv \frac{1}{2 \pi\left(1+R^{2}\right)^{1 / 2}} \int e^{i \tilde{\xi}_{T} \cdot \tilde{k}_{T}-\beta\left(\tilde{\xi}_{T}\right)\left(1+R^{2}\right)^{1 / 2}+\tilde{\gamma}\left(\tilde{\xi}_{T}\right)} d \tilde{\xi}_{T} \\
=\frac{1}{\left(1+R^{2}\right)^{1 / 2}} \int_{0}^{\infty} J_{0}\left(\tilde{\xi}_{T} \tilde{k}_{T}\right) e^{-\beta\left(\tilde{\xi}_{T}\right)\left(1+R^{2}\right)^{1 / 2}+\tilde{\gamma}\left(\tilde{\xi}_{T}\right)_{\tilde{\xi}_{T}} d \tilde{\xi}_{T}}
\end{gathered}
$$

is just the Hankel transform of $\left(1+R^{2}\right)^{-1 / 2} \exp \left[-\beta \sqrt{1+R^{2}}+\tilde{\gamma}\right]$. This shows that the acoustic spectrum (5.31) depends on $\nabla_{y_{T}} S$ only through the square root of $\left(\nabla_{\boldsymbol{y}_{\boldsymbol{T}}} S\right) \cdot\left(\nabla_{\boldsymbol{y}_{\boldsymbol{T}}} S\right)=\left[1-M\left(\boldsymbol{y}_{T}\right) \cos \theta\right]^{2} c_{\infty}^{2} / \widetilde{c^{2}}-\cos ^{2} \theta$, which can be negative for sufficiently small $\cos \theta$.

When $\beta$ and $\gamma$ are set equal to

$$
\beta^{2}=\left(\tilde{\xi}_{T}\right)^{2}+\alpha^{(2-s)}\left(\tilde{\xi}_{T}\right)^{S} \geq 0, \quad \tilde{\gamma}^{2}=\alpha^{(2-s)}\left(\tilde{\xi}_{T}\right)^{S} \geq 0
$$

where $0<s<2$ and $\alpha$ are real parameters, the span-wise component of (6.29) behaves like

$$
R_{i j k l}^{M}\left(0, \tilde{\xi}_{T}, 0\right)=\mathrm{a}_{0,0} e^{-X\left(0, \tilde{\xi}_{T}, 0\right)+\tilde{\gamma}\left(\tilde{\xi}_{T}\right)} \rightarrow \mathrm{a}_{0,0} e^{-\tilde{\xi}_{T}^{(2-s / 2)} / 2 \alpha^{(1-s / 2)}} \text { as } \tilde{\xi}_{T} \rightarrow 0(6.40)
$$

and equation (6.38) becomes

$$
G_{0}\left(\tilde{k}_{T}, R\right)=\frac{H\left(\frac{Z^{(2-S)}}{1+R^{2}}, B, Z\right)}{\left(1+R^{2}+\tilde{k}_{T}^{2}\right)^{3 / 2}}
$$


where

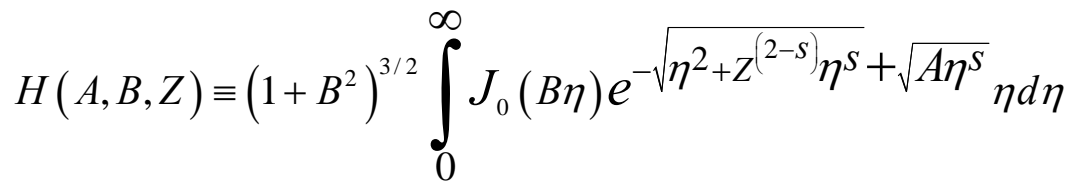

$$
\begin{aligned}
& Z \equiv \alpha\left(1+R^{2}\right)^{1 / 2}, \\
& B^{2} \equiv \frac{\tilde{k}_{T}^{2}}{1+R^{2}},
\end{aligned}
$$

Gradshteyn \& Ryzhik (p.712, \# 6.623, 2 (1965)) show that $H\left(Z^{(2-S)} /\left(1+R^{2}\right), B, Z\right)=1$, when $Z=0$ in which case (6.35) simplifies to

$$
\Psi_{i j k l}\left(k_{1}, \boldsymbol{k}_{T}, \omega\right)=\frac{6 \pi l_{1} l_{T}^{2}}{\lambda} \sum_{m, l=0}^{\infty} a_{m, l}(-1)^{m+l} D_{k_{1}}^{m} D_{\omega}^{l} \frac{1}{\left(1+R^{2}+\tilde{k}_{T}^{2}\right)^{5 / 2}}
$$

Unfortunately, equation (6.42) can be very difficult to evaluate in the general case where $Z \neq 0$, but since $\alpha$ will always be very small, of the order of 0.3 , $Z^{(2-S)} /\left(1+R^{2}\right)=\alpha^{(2-S)} /\left(1+R^{2}\right)^{S / 2}$ will certainly be small as well and $H\left(Z^{(2-S)} /\left(1+R^{2}\right), B, Z\right)$ can be approximated by

$$
H\left(Z^{(2-s)} /\left(1+R^{2}\right), B, Z\right) \approx H(0, B, Z)
$$

Changing the integration variable to $Z^{(2 / s-1)} \eta$ in (6.42) shows that $H(0, B, Z)$ becomes independent of $B$ when $Z$ becomes large. Then since $H(0, B, Z)$ is independent of its second argument for both small and large values of $Z$, and since $\tilde{k}_{T}$, which is proportional to the turbulence Mach number, is usually fairly small compared to $R$, it seems reasonable to approximate $H\left(Z^{(2-S)} /\left(1+R^{2}\right), B, Z\right)$ by

$$
H\left(Z^{(2-s)} /\left(1+R^{2}\right), B, Z\right) \approx H(0,0, Z)=\int_{0}^{\infty} e^{-\sqrt{\eta^{2}+Z^{(2-s)} \eta^{s}}} \eta d \eta,
$$

which depends on the single argument $Z$ and is much easier to evaluate. Equation (6.35) then becomes 


$$
\begin{aligned}
& \Psi_{i j k l}\left(k_{1}, \boldsymbol{k}_{T}, \omega\right) \approx \\
& \frac{6 \pi l_{1} l_{T}^{2}}{\lambda} \sum_{m, l=0}^{\infty} a_{m, l}(-1)^{m+l} D_{k_{1}}^{m} D_{\omega}^{l} \frac{1}{\left(1+R^{2}+\tilde{k}_{T}^{2}\right)^{3 / 2}}\left[\frac{H(0,0, Z)}{1+R^{2}+\tilde{k}_{T}^{2}}-\frac{\alpha^{2}}{3 Z} \frac{d H(0,0, Z)}{d Z}\right]
\end{aligned}
$$

And since

$$
H(0,0, Z) \rightarrow \frac{2}{s \mathrm{Z}^{2(2 / s-1)}} \Gamma\left(\frac{4}{s}\right) \quad \text { as } \quad Z \rightarrow \infty
$$

it follows that

$$
\begin{aligned}
& \Psi_{i j k l}\left(k_{1}, \omega\right) \rightarrow \\
& =\frac{2 \pi l_{1} l_{T}^{2}}{\lambda} \frac{2}{s} \Gamma\left(1+\frac{4}{s}\right) \alpha^{2(2-1 / s)} \sum_{m, l=0}^{\infty} a_{m, l}(-1)^{m+l} D_{k_{1}}^{m} D_{\omega}^{l} \frac{1}{\left(1+R^{2}+\tilde{k}_{T}^{2}\right)^{5 / 2}\left(1+R^{2}\right)^{(2 / s-1)}} \\
& \text { as } Z \rightarrow \infty
\end{aligned}
$$

When $s=1$, equation (6.47) can be put into the form

$$
H(0,0, Z)=1-\left(\frac{\mathrm{Z}}{2}\right)^{2} \int_{0}^{\infty} e^{-(Z / 2) \eta} \frac{\eta}{\sqrt{\eta^{2}+1}} d \eta
$$

which can be expressed in terms of the Struve and Bessel functions $\boldsymbol{H}_{1}(\hat{Z})$ and $N_{1}(\hat{Z})$, respectively, since (Gradshteyn \& Ryzhik (1965), p.316, \#3.366, 3)

$$
\int_{0}^{\infty} e^{-\eta \hat{Z}} \frac{\eta}{\sqrt{\eta^{2}+1}} d \eta=\frac{\pi}{2}\left[\boldsymbol{H}_{1}(\hat{Z})-N_{1}(\hat{Z})\right]-1
$$

and it follows from (Abramowitz \& Stegun, 1965, pp.361, $496,497, \# s 9.1 .30,12.1 .13,12.1 .29)$ that all of the higher order terms in (6.48) can be expressed as higher order Struve and Bessel functions $\boldsymbol{H}_{n}(\hat{Z})$ and $N_{n}(\hat{Z}), n=2,3, \ldots$ plus simple polynomials, which means that it is essentially given in closed form for this case. But the result can be 
rapidly computed even in the more general case where $s$ is not equal to 1 , by simply tabulating $H(0,0, Z)$ against its single argument $Z$.

All of our computations were carried out with $s$ set equal to 1 , in which case (6.40) implies that the transverse correlation tensor $R_{i j k l}^{M}\left(y, 0, \xi_{T}, 0\right)$ behaves like $\exp \cdot \frac{1}{2}\left(\tilde{\xi}_{T} / \alpha^{1 / 3}\right)^{3 / 2}$ and, therefore, has zero slope but large curvature at this point. Figure 4 shows the predicted density-weighted fourth order correlation $R_{1111}^{M}\left(y, 0, \xi_{T}, 0\right)$ based on the functional form (6.39) with the $s, \alpha$ values of $1,0.1$ that were used in the actual acoustic computations. It also shows the corresponding quantity inferred from the Bridges and Podboy (1999) measurements of the second-order correlations by using a locally homogeneous, quasi-normal approximation. The measuring point was on the nozzle lip line 7.5 diameters downstream of the exit in a $M_{J}=0.9$ jet.

\section{Application to a Round Jet}

Current aircraft are usually configured with circular engine exhaust streams. $\mathrm{We}$, therefore, make the present results more concrete by considering the case of an axisymmetric mean flow, where $r_{\perp} \equiv\left|\mathbf{y}_{T}\right|, \quad \cos \varphi=y_{2} / r_{\perp}$,

$$
M=M\left(r_{\perp}\right), \quad \widetilde{c^{2}}=\widetilde{c^{2}}\left(r_{\perp}\right) \text {. The coefficients in equations (4.21) are expressed in }
$$

polar coordinates in Appendix $\mathrm{G}$ and the linear operator in (4.21) now becomes

$$
\mathcal{L}_{k}=\frac{1}{r_{\perp}} \frac{\partial}{\partial r_{\perp}} \frac{r_{\perp} \widetilde{c^{2}}}{(k U-\omega)^{2}} \frac{\partial}{\partial r_{\perp}}-\frac{\widetilde{c^{2}}}{(k U-\omega)^{2}}\left(k^{2}-\frac{1}{r_{\perp}^{2}} \frac{\partial^{2}}{\partial \varphi^{2}}\right)+1 \text {. }
$$

which implies that $\hat{G}_{o}$ will be of the form

$$
\hat{G}_{o}=\sum_{n=-\infty}^{\infty} e^{i n\left(\varphi-\varphi^{\prime}\right)} G^{(n)}\left(r_{\perp} \mid r_{\perp}^{\prime}\right)
$$

where

$$
\mathcal{L}_{n k} G^{(n)}=\frac{\delta\left(r_{\perp}-r_{\perp}^{\prime}\right)}{(2 \pi)^{3} r_{\perp}}
$$

and

$$
\mathcal{L}_{n k} \equiv \frac{1}{r_{\perp}} \frac{d}{d r_{\perp}} \frac{r_{\perp} \widetilde{c^{2}}}{(k U-\omega)^{2}} \frac{d}{d r_{\perp}}+\left[1-\frac{\widetilde{c^{2}}}{(k U-\omega)^{2}}\left(k^{2}+\frac{n^{2}}{r_{\perp}^{2}}\right)\right]
$$

It follows that 


$$
G^{(n)}=\frac{w_{n}^{(1)}\left(r_{\perp}, k\right) w_{n}^{(2)}\left(r_{\perp}^{\prime}, k\right)}{\Delta_{n}(k)} \quad(\text { no sum on } n)
$$

for $r_{\perp}>r_{\perp}^{\prime}$ (and a similar equation with $r_{\perp}$ and $r_{\perp}^{\prime}$ interchanged when $r_{\perp}<r^{\prime}$ ), where

$$
\begin{gathered}
\mathcal{L}_{n k} w_{n}^{(l)}\left(r_{\perp}, k\right)=0 \quad \text { for } l=1,2 \quad \text { (no sum on } n \text { ) } \\
w_{n}^{(1)} \sim \frac{1}{\sqrt{r_{\perp}}} e^{-\sqrt{k^{2}-\left(\omega / c_{\infty}\right)^{2}} r_{\perp}} \quad \text { as } r_{\perp} \rightarrow \infty \\
w_{n}^{(2)} \rightarrow r_{\perp}^{|n|} \quad \text { as } r_{\perp} \rightarrow 0
\end{gathered}
$$

and

$$
\Delta_{n} \equiv-(2 \pi)^{3} r_{\perp} \widetilde{c^{2}} W_{n} /(k U-\omega)^{2}
$$

where the Wronskian

$$
\left.W_{n} \equiv w_{n}^{(1)} \frac{d}{d r_{\perp}} w_{n}^{(2)}-w_{n}^{(2)} \frac{d}{d r_{\perp}} w_{n}^{(1)}, \quad \text { (no sum on } n\right)
$$

of $w_{n}^{(1)}$ and $w_{n}^{(2)}$, is independent of $r_{\perp}$. It follows from (4.17) and (4.21) that

$$
\widetilde{\mathcal{G}_{0}}=\sum_{n=-\infty}^{\infty} \frac{e^{i n\left(\varphi-\varphi^{\prime}\right)}}{\Delta_{n}} w_{n}^{(2)}\left(r_{\perp}^{\prime}, \frac{\omega}{c_{\infty}} \cos \theta\right)
$$

Since the spectral functions are expected to be independent of $\varphi^{\prime}$ in equation(6.27), it is appropriate to average it over this angle. Appendix $G$ and equations (C.18) to (C.22) can be used to show that the result is given by

$$
\frac{1}{2 \pi} \int_{0}^{2 \pi} I_{\omega} d \varphi^{\prime}=\left(\frac{2 \pi}{x}\right)^{2} \frac{2 \pi \omega^{4} \sin \theta}{c_{\infty}} \sum_{n=-\infty}^{n=\infty} I_{n}
$$

where

$$
\begin{gathered}
I_{n} \equiv\left|F_{n}\left[1+w^{\prime}\left(z_{0}\right)\right]\right|^{2}\left[\Phi{ }^{*}{ }_{1111}\left|C_{c}\right|^{2}+2 \mathcal{R e}\left[C_{c}\left(1-C_{c}^{*}\right)\right] \Phi{ }^{*}{ }_{1122}+\left|1-C_{c}\right|^{2} \Phi^{*}{ }_{2222}\right] \\
+4 B_{n} \Phi^{*}{ }_{1212}+2 E_{n}\left(\Phi^{*}{ }_{2222}-\Phi^{*}{ }_{2233}\right)
\end{gathered}
$$

with $C_{c}$ given by (6.16), 


$$
\begin{aligned}
& \Phi *_{i j k l} \equiv \Phi{ }_{i j k l}\left(\mathbf{y} ; \frac{\omega}{c_{\infty}} \cos \theta, \omega\left(1-M_{c} \cos \theta\right)\right) \\
& F_{n} \equiv \frac{w_{n}^{(2)}}{\Delta_{n} \omega^{3 / 2}}, \\
& B_{n} \equiv\left[\left|\frac{\omega \sqrt{\widetilde{c^{2}}} \cos \theta}{c_{\infty} \bar{D}}\left(\frac{F_{n} \sqrt{\widetilde{c^{2}}}}{\bar{D}}\right)^{\prime}-\frac{(\gamma-1) F_{n} c_{\infty} M^{\prime}}{2 \bar{D}}\right|^{2}+\frac{n^{2} C^{2} \widetilde{c^{2}}}{r_{\perp}^{2}}\left|\frac{F_{n}}{\bar{D}}\right|^{2}\right]\left|z_{0}^{3} w^{\prime \prime}\left(z_{0}\right)\right|^{2} \\
& E_{n} \equiv \\
& {\left[\left|\frac{\widetilde{c^{2}}}{\bar{D}^{2}}\left(\frac{F_{n}^{\prime}}{r_{\perp}}-\frac{n^{2} F_{n}}{r_{\perp}^{2}}\right)+\frac{\left(1-C^{2}\right) F_{n}}{2}\right|^{2}+\frac{\widetilde{c^{2}} n^{2}}{\bar{D}^{2}}\left|\left(\frac{F_{n} \sqrt{\widetilde{c^{2}}}}{r_{\perp} \bar{D}}\right)^{\prime}\right|^{2}-\frac{\left(1-C^{2}\right)^{2}\left|F_{n}\right|^{2}}{4}\right]\left|z_{0}^{3} w^{\prime \prime}\left(z_{0}\right)\right|^{2}}
\end{aligned}
$$

and

$$
\bar{D} \equiv \omega(1-M \cos \theta)
$$

where the Doppler weighted cosine function $C_{c}$ is defined by (6.16) with $C$ given by (C.4) and the replacements (5.38) and (5.39) inserted after the critical layer disappears.

\section{Prediction of the Sound Field and Comparison with Measurements}

In order to use the point-wise acoustic spectra (7.12)-(7.17) to predict the radiated sound field it is necessary to relate the modified spectra $\Phi_{i j k l}\left(\mathbf{y} ; \frac{\omega}{c_{\infty}} \cos \theta, \frac{\omega}{c_{\infty}} \nabla_{\mathbf{y}} S, \omega\left(1-M_{c} \cos \theta\right)\right)$ to the ordinary moving frame spectra $\Psi_{i j k l}\left(\mathbf{y} ; \frac{\omega}{c_{\infty}} \cos \theta, \frac{\omega}{c_{\infty}} \nabla_{\mathbf{y}} S, \omega\left(1-M_{c} \cos \theta\right)\right)$ by inserting (5.13) into the basic definitions (5.29) and (6.1). Appropriate special cases of the source model(6.47) and (6.48) can then be inserted into the result.

The simplest formulas that are consistent with the Bridges \& Podboy (1999) measurements correspond to setting the integer $s$ equal to 1 in (6.47) and 
retaining only the lowest-order source non-compactness term, i.e., the $a_{1,0}$ term. . Then since

$$
k_{1} \frac{\partial}{\partial k_{1}}=\frac{\tilde{k}_{1}^{2} \alpha^{2}}{Z} \frac{\partial}{\partial Z}
$$

equation (6.48) reduces to

$$
\begin{aligned}
& \Psi_{i j k l}\left(k_{1}, \boldsymbol{k}_{T}, \omega\right)=\frac{2 \pi l_{1} l_{T}^{2}}{\lambda\left(1+R^{2}+\tilde{k}_{T}^{2}\right)^{3 / 2}}\left\{\left(a_{0,0}-a_{1,0}\right)\left(\frac{3 H(0,0, Z)}{1+R^{2}+\tilde{k}_{T}^{2}}-\frac{\alpha^{2}}{Z} \frac{d H(0,0, Z)}{d Z}\right)\right. \\
& \left.+a_{1,0} \tilde{k}_{1}^{2}\left(\frac{15 H(0,0, Z)}{\left(1+R^{2}+\tilde{k}_{T}^{2}\right)^{2}}-\left[\frac{\alpha^{4}}{Z^{3}}+\frac{6 \alpha^{2}}{Z\left(1+R^{2}+\tilde{k}_{T}^{2}\right)}\right] \frac{d H(0,0, Z)}{d Z}+\frac{\alpha^{4}}{Z^{2}} \frac{d^{2} H(0,0, Z)}{d Z^{2}}\right)\right\}
\end{aligned}
$$

where $H(0,0, Z)$ is given by $(6.51)$.

Historically, the convection Mach number $M_{c}$ has typically been set equal to $0.68 U_{J} / c_{\infty}$ (where $U_{J}$ denotes the mean jet exit velocity)—which would certainly be reasonable upstream of the end of the potential core, but would not make sense downstream of this point. We therefore generalize this result by setting

$$
M_{c}=0.68 U_{c / l} / c_{\infty}
$$

where $U_{c / l}$ denotes the centerline velocity.

Equation (7.12) can now be summed over the noise producing region of the jet to predict the radiated sound. But, as with any acoustic analogy approach, this requires information about the mean flow and turbulence statistics, which ultimately has to be obtained from measurements of the flow, which limit the applicability of the result to a limited range of operating conditions. But it is necessary to extrapolate the experimental results beyond the available data base in order to extend this approach to a broad range of operating conditions. We do this using the experimental results to relate the source model parameters to the $k-\varepsilon$ length, time and velocity scales, $k^{3 / 2} / \varepsilon, k / \varepsilon$ and $k^{1 / 2}$,respectively. We then use the NASA Glenn version of the NPARC Wind code (Nelson and Power, 2001) to determine the mean flow along with the spatial distribution of the length, time and velocity scales within the jet along with the dimensionless time scale parameter $k U^{\prime} / \varepsilon$, where the prime is meant to denote differentiation with respect to $r_{\perp}$ in the present context. More specifically, we assume that the length, time and velocity scales $l_{1}, l_{T}, \lambda^{-1}$ and $a_{0,0}$ that appear in equation (6.48) (directly and 
implicitly through equations(6.37) and(6.43)) are proportional to the $k-\varepsilon$ length, time and kinetic energy scales $k^{3 / 2} / \varepsilon, k / \varepsilon$ and $(\bar{\rho} k)^{2}$ respectively, i.e. we put

$$
l_{1} \approx C_{1} k^{3 / 2} / \varepsilon, \quad l_{T} \approx C_{T} k^{3 / 2} / \varepsilon, \quad \lambda^{-1} \approx C_{\tau} k / \varepsilon, a_{0,0}=R_{i j k l}(\boldsymbol{y}, \boldsymbol{0}, 0) \approx C_{i j k l}(\bar{\rho} k)^{2}
$$

where the dimensionless parameters $C_{1}, C_{T}, C_{\tau}$ and $C_{i j k l}$ can, in general, depend on the dimensionless time scale parameter $k\left|U^{\prime}\right| / \varepsilon$. The first three of these are determined with reference to the integral length and time scales measured by (Bridges \& Pobody,1999), and the last is inferred from their mean square fluctuating velocity measurements. As noted in section $6, \Psi_{1212}$ depends on the autocovariance of the cross velocity components, while the remaining spectral components in the acoustic formulas depend only on the covariances of the squares of velocity components. We, therefore, expect the parameters in the former to scale somewhat differently than those in the latter.

Since RANS-type models are based on the assumption that the turbulence is close to equilibrium, we expect the dimensionless parameters $C_{1}, C_{T}, C_{\tau}$ and $C_{i j k l}$ to be relatively constant in the initial mixing layers and fully developed region further downsteam where the flow evolves fairly slowly in the streamwise direction (Pope,2000, p.362 \& p. 365) —but not necessarily in the transition region, where the flow can be fairly far from equilibrium (see figure 2). We account for variations in the latter region by allowing these parameters to depend on $k\left|U^{\prime}\right| / \varepsilon$. A comparison of the experimentally measured length, time and kinetic energy scales with the corresponding RANS-derived quantities showed that the data could be reasonably well represented by using constant values of $C_{1}, C_{T}, C_{i j k l}$ everywhere in the flow, but that $C_{\tau}$ would have to depend on $k\left|U^{\prime}\right| / \varepsilon$ in the transition region. Figure 5 shows results obtained by plotting the ratio of the experimentally measured integral time scale obtained from auto-correlations of the streamwise velocity fluctuations to the $k / \varepsilon$ RANS based scale vs. $k\left|U^{\prime}\right| / \varepsilon$ for a $M_{J}=0.9$ jet. These scales are directly proportional to one another in the fully developed region and in the initial shear layer, which maps into a single point in this plot because $k\left|U^{\prime}\right| / \varepsilon$ turns out to be nearly constant there. The time scale $\lambda^{-1}$ can, therefore, be made to transition from its initial shear layer behavior to its behavior in the fully developed region by putting $C_{\tau}=C_{\tau}^{(0)} F\left(k\left|U^{\prime}\right| / \varepsilon\right)$. We use $C_{\tau}^{(0)}=0.7$ in $\Psi_{1212} C_{\tau}^{(0)}=0.31$ for all the other spectral components.

As noted in section 6.2 , the individual $a_{0,0}$ values are equal to the densityweighted averages of the appropriate velocity components, and it turns out that the ones that actually appear in the final formulas only involve squares of these components. Lacking any information about the $4^{\text {th }}$ order correlations we assume quasi-normality and infer from equation (6.17) that the dimensionless $C_{i j k l}$ can be express expressed entirely in terms of these quantities, or more specifically that 
$C_{1111}=2\left(\overline{v_{1}^{\prime 2}}\right)^{2} ; C_{2222}=C_{3333}=C_{2233}=2\left(\overline{v_{2}^{\prime 2}}\right)^{2} ; C_{1122}=C_{1133}=C_{1212}=2 \overline{v_{1}^{\prime 2}} \frac{1}{v_{2}^{\prime 2}}$, where $\boldsymbol{v}^{\prime}=\left\{v_{1}^{\prime}, v_{2}^{\prime}, v_{3}^{\prime}\right\}$. The last relation follows from equation(6.19) which implies that $\left[R_{12}^{M}(\boldsymbol{y}, \boldsymbol{0}, 0)\right]^{2}=R_{11}^{M}(\boldsymbol{y}, \boldsymbol{0}, 0) \times R_{22}^{M}(\boldsymbol{y}, \boldsymbol{0}, 0)$ since $R_{1212}^{M}(\boldsymbol{y}, \boldsymbol{0}, 0)$ and $R_{1122}^{M}(\boldsymbol{y}, \boldsymbol{0}, 0)$ must be equal by definition. The mean square velocity components are now assumed to be proportional to the turbulence kinetic energy distribution with the best overall fit to the data corresponding to the relative proportionality $\overline{v_{1}^{\prime 2}}=0.8 \mathrm{k}$ and $\overline{v_{2}^{\prime 2}}=\overline{v_{3}^{\prime 2}}=0.6 \mathrm{k}$. The anisotropy in the turbulent kinetic energy has a strong effect on the $90^{\circ}$ degree spectral shape because there are strong cancellations among the various components of the spectra $\Psi_{i j k l}^{*}$ of the generalized Reynolds stress autocovariance tensor that contribute to the spectra $\Phi_{i j k l}^{*}$ appearing in (6.27).

The (Bridges/Pobody ,1999) data suggest that $C_{1}=0.96, C_{T}=0.144$, for $\Psi_{1212}$ and $C_{1}=0.58, C_{T}=0.186$ for all other spectral components would be reasonable values of the remaining parameters in (8.4). It also seems consistent to put $\alpha=0.2, a_{1,0}=0.972 a_{0,0}$ for $\Psi_{1212}$ and $\alpha=0.34, a_{1,0}=0.54$ for all other components of (8.2). The remaining unknowns in (7.12), including quantities related to the critical layer, depend on the mean flow and can, therefore be determined directly from the RANS calculation.

The WIND code was used to obtain RANS solutions for cold jets with acoustic Mach numbers $M_{J} \equiv U_{J} / c_{\infty}$ of $0.50,0.90$, and 1.4--which span the range of current interest--and upstream nozzle conditions specified in terms of appropriate plenum temperature and pressure ratios. Equations (7.12) to (7.17) were then used along with the parameter choices described above to calculate their far-field acoustic spectra on the arc $x / D_{J}=100$.

Figure 6 is a comparison of the results with narrow band data obtained from the NASA Glenn SHJAR rig (Khavaran et. al., 2002) for the same upstream conditions. No vertical adjustments were made to obtain the best fit. Atmospheric attenuation was removed from the measurements in order to make lossless comparisons with the predictions. The overall agreement appears to be quite good but there is a tendency to under predict the higher frequency components of the $90^{\circ}$ spectrum in the supersonic case. This is because the flow is not correctly expanded in this case and the present analysis does not account for the resulting shock associated noise (shown cross hatched in Figure 6c). Another significant discrepancy is in the prediction of the $\theta=30^{\circ}$ spectrum for $M_{J}=0.9$ jet (figure 6e). This may, in part, be due to the breakdown of the weakly non-parallel flow approximation in the region near the end of the potential core - which is an important source region for this transonic Mach number. Notice that the spectra tend to be narrower and more highly peaked at $\theta=30^{\circ}$ than at $\theta=90^{\circ}$, especially at the higher Mach numbers. Figure 7 is a comparison of the present result with the best previous results obtained from the Glenn JeNo code, which is based on 
a parallel model (with an ad hock assumption used to eliminate the critical layer singularity) and does not account for the strong retarded time variations produced by the long range correlations.

\section{Discussion}

The acoustic predictions of the last section were based on the special case (7.12) of the more general result(6.27) which, as pointed out at the end of section 6.1 , is composed of three distinct terms. The first of these (referred to as term $I$ ) is the product involving the first term in square brackets. It is not hard to see that it exhibits the usual quadrupole- like behavior at low frequencies, i.e. it behaves like $\omega^{4}$ as $\omega \rightarrow 0$. The second term (referred to as term II) is the product involving $\Phi_{1212}^{*}$. It exhibits dipole- like behavior at low frequencies, i.e. it behaves like $\omega^{2}$ as $\omega \rightarrow 0$. It should not, however, be interpreted as an actual dipole source, since it is now known (Goldstein (1975), Balsa (1977), Musafir (1992) and Afsar et al (2007)) that the mean flow can raise the efficiency of certain components of the quadrupole source from $\omega^{4}$ to $\omega^{2}$ as $\omega \rightarrow 0$. Afsar et al (2007) even show that, these components exhibit the same inverse six Doppler factor singularity as term II, which as noted above, corresponds to the strongest critical layer singularity in the present result . So, while the Goldstein (1975) and Afsar et al (2007) result shows that term $I I$ is the dominant small angle noise source at low frequencies, the present result shows that this term is the dominant small angle supersonic noise source at all frequencies. The remaining term does not appear to make a significant contribution to the sound field for any of the conditions considered and will, therefore, not be discussed any further.

Term $I I$ only contributes to the $90^{\circ}$ sound field at extremely low frequencies, but becomes increasingly important at small angles as the acoustic Mach number increases. Each of the three terms acts like a statistically independent sound source. Their individual contributions to the overall $30^{\circ}$ spectrum are shown in figure 8. Term $I I I$ is too small to show up on the figure. Notice that Term $I I$ is only dominant at relatively low frequencies when $M_{J}$ is equal to 0.5 , but becomes dominant at all frequencies when the Mach number is supersonic. This occurs because the six powers of the Doppler factor that appears in the first member of this term causes it to be very large.

Figure 9 shows the contribution of various axial slices of the jet to the overall spectra for the supersonic case. Notice that these point-wise spectra tend to be much narrower at $\theta=30^{\circ}$ than at $\theta=90^{\circ}$--especially in the upstream region. This is, in part, due to the fact that much of the small angle sound is generated in the critical layer while none of the $90^{\circ}$ sound is generated there.

For acoustic Mach number is close to unity, much of the small angle radiation is generated near the end of the potential core --which may explain the tendency to under predict the peak frequency of the $30^{\circ}$ spectrum in the $M_{J}=0.9$ case, since the weakly non-parallel flow assumption tends to breakdown in this region. But this may also be caused by the break down in the quasi-equilibrium assumption (on which the RANS modeling is based) in this region. These 
difficulties can probably be overcome by using a hybrid RANS/LES approach of the type described by Goldstein (2006), but that would greatly increase the expense of the computation.

The spectra of each of the three components of (6.27) is determined from(8.2), but with different coefficients for each component. The 2 nd term in the curly brackets of this latter equation is associated with source non-compactness effects and does not contribute to the $90^{\circ}$ sound field. It is also relatively insignificant at all angles when $M$ becomes small but becomes the dominant source of the small angle sound at $M_{J}=1.4$. This term introduces an $\omega^{2}$ factor which causes term $I I$ to behave more like term $I$ at high Mach numbers.

Computations were only carried out for subsonic convection Mach numbers which correspond to the relatively low $(<1.5)$ acoustic Mach numbers where nonlinear propagation effects and Mach wave radiation are believed to be unimportant. The instability wave contribution to the Green's function is then negligibly small and the convective amplification of the sound sources turns out to be relatively insignificant compared to the very strong critical layer effects produced by the mean flow Doppler factor. Fortunately, this also corresponds to the Mach number range of most technological interest.

The critical layer only appears when the mean flow acoustic Mach number is supersonic and the observation angle (as measured from the downstream axis) is fairly small $\left(<45^{\circ}\right)$. It gradually moves inboard from the nozzle lip line with increasing downstream distance until it reaches a point beyond the end of the potential core where it quickly moves onto the jet axis and suddenly disappears. But the propagator is still very close to being singular, and can consequently be relatively large, for a significant distance downstream of this point. It was, therefore, necessary to construct a new inner solution for this region as wellespecially since much of the small angle sound field is actually generated in this relatively localized portion of the jet when the acoustic Mach number is close to unity. This tends to decrease the width of the corresponding acoustic spectrum because the band width of the turbulent source tends to be relatively small in each streamwise slice of the jet as compared to the relatively large frequency variation that occurs over the entire jet.

The source distribution for the larger angle acoustic radiation tends to be more evenly distributed over the jet (since no singular layer occurs at these angles). This contributes to making the large angle acoustic spectrum more broadband than the small angle spectrum when the jet acoustic Mach number is close to one, but the relative spectral width of the individual slice contributions is probably the more important factor here.

The upstream critical layers move into the high turbulence region along the nozzle lip with increasing jet Mach number. This causes more of small angle radiation will be generated in these layers, which generate narrower spectra than the reminder of the jet when the correct inner solutions are used in these regions. This partially explains why the width of the small angle spectra 
decreases with jet Mach number. It is, of course, well known that Doppler frequency shift and mean-flow refraction effects also play an important role here.

This work was carried out under the NASA Glenn IR\&D program. The authors would like to thank Dr. Anthony Strazisar (the Glenn Chief Scientist) and the Glenn Research Advisory Board for supporting this work. We would like to thank Dr.James Bridges for his support and helpful discussion of the experimental results, Dr. Nicholas Georgiadis for running the WIND code, and Dr. Abbas Khavaran for supplying the JeNo results. We would also like to thank an anonymous referee for pointing out the connection between the dominant small angle noise source in the present work and the earlier low frequency results of Afsar, et al (2007) and Goldstein (1975).

\section{Appendix A Mean Flow}

As in Goldstein \& Leib (2005), we suppose that all lengths have been normalized by some characteristic cross flow dimension of the jet (say its diameter, in the case of a round jet) and all velocities by some appropriate characteristic streamwise velocity with similar obvious normalization for the density, pressure and temperature. Then the mean flow variables and stresses should expand like

$$
\begin{gathered}
\tilde{v}_{1}=U\left(Y, \boldsymbol{y}_{T}\right)+\varepsilon U^{(1)}\left(Y, \boldsymbol{y}_{T}\right)+\ldots \\
\tilde{\boldsymbol{v}}_{T}=\varepsilon \boldsymbol{V}\left(Y, \boldsymbol{y}_{T}\right)+\varepsilon^{2} \boldsymbol{V}^{(1)}\left(Y, \boldsymbol{y}_{T}\right)+\ldots \\
\bar{p}_{e}=P\left(Y, \boldsymbol{y}_{T}\right)+\varepsilon P^{(1)}\left(Y, \boldsymbol{y}_{T}\right)+\varepsilon^{2} P^{(2)}\left(Y, \boldsymbol{y}_{T}\right)+\ldots \\
\bar{\rho}=\bar{R}\left(Y, \boldsymbol{y}_{T}\right)+\varepsilon R^{(1)}\left(Y, \boldsymbol{y}_{T}\right)+\ldots
\end{gathered}
$$

and

$$
\overline{e_{\mu j}^{\prime}} \equiv-\bar{\rho} \widetilde{v_{\mu}^{\prime} v_{j}^{\prime}}+\frac{\gamma-1}{2} \delta_{v j} \bar{\rho} \widetilde{v^{\prime 2}}+\bar{\sigma}_{v j}=T_{\mu j}^{(0)}+\varepsilon T_{\mu j}^{(1)}+\varepsilon^{2} T_{\mu j}^{(2)}+\ldots,
$$

where

$$
Y \equiv \varepsilon y_{1}
$$

denotes the slow streamwise "source" variable, and

$$
\boldsymbol{y}_{T}=\left\{y_{2}, y_{3}\right\}
$$




$$
\tilde{\boldsymbol{v}}_{T}=\left\{\tilde{v}_{2}, \tilde{v}_{3}\right\}
$$

denote cross flow variables.

Substituting these into the mean flow equations (2.7) to(2.9) (written in terms of the "source" variable $y$ ) and assuming that the generalized Reynolds stresses vanish in the free stream, shows that the result will only balance if

$$
\begin{gathered}
T_{1 j}^{(0)}=T_{j 1}^{(0)}=T_{4 j}^{(0)}=0 \quad \text { for } j=2,3 \\
\frac{\partial}{\partial y_{j}}\left(P-T_{j j}^{(0)}\right)=\frac{\partial}{\partial y_{l}} T_{j l}^{(0)} \quad(\text { no sum on } j \neq l=2,3)
\end{gathered}
$$

and

$$
\frac{\partial}{\partial y_{j}}\left(P^{(1)}-T_{j j}^{(1)}\right)=\frac{\partial}{\partial y_{l}} T_{j l}^{(1)} \quad(\text { no sum on } j \neq l=2,3)
$$

The lowest order mean flow equations become

$$
\begin{gathered}
D_{o} \bar{R}=0 \\
D_{o} \bar{R} U=S \\
D_{o} \bar{R} V_{j}+\frac{\partial P^{(2)}}{\partial y_{j}}=\frac{\partial}{\partial Y} T_{j 1}^{(1)}+\frac{\partial T_{j l}^{(2)}}{\partial y_{l}}, \text { for } j, l=2,3,
\end{gathered}
$$

and

$$
\begin{aligned}
D_{0}\left(\frac{\gamma}{\gamma-1} P+\frac{1}{2} \bar{R} U^{2}\right) & =\frac{\partial}{\partial Y}\left[\frac{T_{41}^{(0)}}{\gamma-1}+U T_{11}^{(0)}+\frac{1}{2} U\left(T_{11}^{(0)}+T_{l l}^{(0)}\right)\right] \\
& +\frac{\partial}{\partial y_{j}}\left[\frac{T_{4 j}^{(1)}}{\gamma-1}+U T_{j 1}^{(1)}+V_{l} T_{j l}^{(0)}+\frac{1}{2} V_{j}\left(T_{11}^{(0)}+T_{l l}^{(0)}\right)\right] \text { for } j, l=2,3,
\end{aligned}
$$

where the operator $D_{0}$ is now given by

and we have put

$$
D_{0}=\frac{\partial}{\partial Y} U+\frac{\partial}{\partial y_{j}} V_{j} \quad \text { for } j=2,3
$$




$$
S \equiv \frac{\partial}{\partial Y}\left(T_{11}^{(0)}-P\right)+\frac{\partial}{\partial y_{j}} T_{1 j}^{(1)}, \quad \text { for } j=2,3 .
$$

Equations(2.21), (A.3) and (A.5) imply that

$$
\tilde{\theta}_{i j}=\delta_{i j}\left(P+\varepsilon P^{(1)}\right)-\left(T_{i j}^{(0)}+\varepsilon T_{i j}^{(1)}\right)+0\left(\varepsilon^{2}\right)
$$

and it therefore follows from (A.10) and (A.11) that

$$
\frac{\partial \tilde{\theta}_{i j}}{\partial y_{j}}=-\varepsilon \delta_{i 1} S+0\left(\varepsilon^{2}\right)
$$

\section{Appendix B Details of Critical Layer Solution}

Equation (4.35) can be written as

$$
\left(V_{c}-U_{c} \frac{\partial y_{c}}{\partial Y}\right)\left(\frac{\partial}{\partial \bar{y}_{\perp}}-2 \alpha^{2} \bar{y}_{\perp}\right) g_{54}^{0}=\left[U_{c}\left(\frac{\partial U}{\partial Y}\right)_{c}+V_{c} U_{c}^{\prime}\right] g_{14}^{0}
$$

or since the chain rule implies that

$$
U_{c}^{\prime} \frac{\partial y_{c}}{\partial Y}+\left(\frac{\partial U}{\partial Y}\right)_{c}=\frac{d U\left(y_{c}(Y), Y\right)}{d Y}=0
$$

as

$$
\left(\frac{\partial}{\partial \bar{y}_{\perp}}-2 \alpha^{2} \bar{y}_{\perp}\right) g_{54}^{0}=U_{c}^{\prime} g_{14}^{0}
$$

And it, therefore, follows that

$$
\begin{aligned}
g_{54}^{0} & =-2 \alpha^{2} \widetilde{c_{c}^{2}} \hat{a}_{4} \exp \cdot\left(\alpha^{2} \bar{y}_{\perp}^{2}\right) \int_{\bar{y}_{\perp}}^{\infty} \exp \cdot\left(-\alpha^{2} \eta^{2}\right) \frac{\partial}{\partial \eta} \exp \cdot\left(\alpha^{2} \eta^{2}\right) \int_{\eta}^{\infty} \exp \cdot\left(-\alpha^{2} \bar{\eta}^{2}\right) d \bar{\eta} d \eta \\
& =2 \alpha^{2} \widetilde{c_{c}^{2}} \hat{a}_{4} \exp \cdot\left(\alpha^{2} \bar{y}_{\perp}^{2}\right) \int_{\bar{y}_{\perp}}^{\infty}\left[1+\alpha^{2}\left(\bar{y}_{\perp}^{2}-\eta^{2}\right)\right] \exp \cdot\left(-\alpha^{2} \eta^{2}\right) d \eta
\end{aligned}
$$




$$
=\frac{\widetilde{c_{c}^{2}} \hat{a}_{4}}{2} \frac{\partial^{2}}{\partial \bar{y}_{\perp}^{2}} \exp .\left(\alpha^{2} \bar{y}_{\perp}^{2}\right) \int_{\bar{y}_{\perp}}^{\infty} \exp .\left(-\alpha^{2} \eta^{2}\right) d \eta \sim \frac{1}{\bar{y}_{\perp}^{3}} \quad \text { as } \overline{\mathrm{y}}_{\perp} \rightarrow \infty
$$

which implies that $\hat{g}_{54}^{a, 0}=0$ in (4.27). Inserting (4.41) into (4.33) shows that $g_{t 4}^{0}$ satisfies

$$
\left(V_{c}-U_{c} \frac{\partial y_{c}}{\partial Y}\right)\left(\frac{\partial}{\partial \bar{y}_{\perp}}-2 \alpha^{2} \bar{y}_{\perp}\right) g_{t 4}^{0}=-\frac{\partial \bar{\gamma}}{\partial s}
$$

which, in view of (4.44) possesses the solution

$$
\begin{aligned}
g_{t 4}^{0}=\frac{-1}{\left(V_{c}-U_{c} \frac{\partial y_{c}}{\partial Y}\right)} \frac{\partial}{\partial s} \hat{a}_{4} \widetilde{c_{c}^{2}}\left(2 \alpha^{2} \bar{y}_{\perp} e^{\alpha^{2} \bar{y}_{\perp}^{2}} \int_{\bar{y}_{\perp}}^{\infty} e^{-\alpha^{2} \eta^{2}} d \eta-1\right) \\
=\frac{-1}{\left(V_{c}-U_{c} \frac{\partial y_{c}}{\partial Y}\right)} \frac{\partial}{\partial s} \widetilde{c_{c}^{2}} \hat{a}_{4} \frac{\partial}{\partial \bar{y}_{\perp}} e^{\alpha^{2} \bar{y}_{\perp}^{2}} \int_{\bar{y}_{\perp}}^{\infty} e^{-\alpha^{2} \eta^{2}} d \eta
\end{aligned}
$$

that matches with the 2 nd equation(4.27). And inserting this along with equations(4.43) and (4.45) into equation(4.34) and using (4.29) and (4.39) shows that

$$
\begin{aligned}
\frac{\partial}{\partial y_{\perp}}\left(g_{\perp 4}^{0}\right. & \left.+\frac{k^{2} \widetilde{c_{c}^{2}}}{\left(V_{c}-U_{c} \frac{\partial y_{c}}{\partial Y}\right)} \hat{a}_{4} e^{\alpha^{2} \bar{y}_{\perp}} \int_{\bar{y}_{\perp}}^{\infty} e^{-\alpha^{2} \eta^{2}} d \eta\right) \\
& =\frac{-1}{\left(V_{c}-U_{c} \frac{\partial y_{c}}{\partial Y}\right) h_{\perp}} \frac{\partial}{\partial s} h_{\perp} \frac{\partial \widetilde{c_{c}^{2}} \hat{a}_{4}}{\partial s} \frac{\partial}{\partial \bar{y}_{\perp}} e^{\alpha^{2} \bar{y}_{\perp}^{2}} \int_{\bar{y}_{\perp}}^{\infty} e^{-\alpha^{2} \eta^{2}} d \eta
\end{aligned}
$$

which, in view of the $2^{\text {nd }}$ equation(4.27), implies that 


$$
g_{\perp 4}^{0}=\frac{-1}{\left(V_{c}-U_{c} \frac{\partial y_{c}}{\partial Y}\right)}\left(\frac{1}{h_{\perp}} \frac{\partial}{\partial s} h_{\perp} \frac{\partial \widetilde{c_{c}^{2}} \hat{a}_{4}}{\partial s}+k^{2} \widetilde{c_{c}^{2}} \hat{a}_{4}\right) e^{\alpha^{2} \bar{y}_{\perp}^{2}} \int_{y_{\perp}}^{\infty} e^{-\alpha^{2} \eta^{2}} d \eta
$$

\section{Appendix C Uniformly Valid Formulas for Propagator Components}

In the "outer region" where the source point, $\boldsymbol{y}_{T}$, is outside of the critical layer, equations(4.21),(5.20) and (5.32) imply that

$$
\begin{aligned}
& \bar{\Gamma}_{i i}\left(\boldsymbol{x} \mid \boldsymbol{y}_{T}\right)=-\widetilde{\mathcal{G}_{0}}\left(\varphi, \frac{\omega}{c_{\infty}} \cos \theta, \omega \mid \boldsymbol{y}_{T}\right) \\
& \bar{\Gamma}_{11}\left(\boldsymbol{x} \mid \boldsymbol{y}_{\perp}\right)=-C^{2} \widetilde{\mathcal{G}_{0}}\left(\varphi, \frac{\omega}{c_{\infty}} \cos \theta, \omega \mid \boldsymbol{y}_{\perp}\right)
\end{aligned}
$$

and

$$
\begin{aligned}
& G_{1 i}=-\frac{i \cos \theta}{2 c_{\infty} \omega}\left[\frac{\widetilde{c^{2}}}{(1-M \cos \theta)^{2}} \frac{\partial \widetilde{\mathcal{G}_{0}}}{\partial y_{i}}+\frac{\partial}{\partial y_{i}} \frac{\widetilde{\mathcal{G}_{0}} \widetilde{c^{2}}}{(1-M \cos \theta)^{2}}\right]+i \frac{\gamma-1}{2 \omega} \frac{\partial U}{\partial y_{i}} \frac{\widetilde{\mathcal{G}_{0}}}{(1-M \cos \theta)} \\
& =-\frac{i}{\omega(1-M \cos \theta)}\left[\frac{\cos \theta \sqrt{c^{2}}}{c_{\infty}} \frac{\partial}{\partial y_{i}} \frac{\widetilde{\mathcal{G}_{0}} \sqrt{\widetilde{c^{2}}}}{(1-M \cos \theta)}-\frac{(\gamma-1)}{2} c_{\infty} \frac{\partial M}{\partial y_{i}} \widetilde{\mathcal{G}_{0}}\right], \quad i=2,3
\end{aligned}
$$

where the Doppler weighted cosine function $C$ is defined by

$$
C^{2} \equiv \frac{\widetilde{c^{2}} \cos ^{2} \theta}{c_{\infty}^{2}\left[M\left(\boldsymbol{y}_{T}\right) \cos \theta-1\right]^{2}}
$$

To obtain the corresponding formulas in the "inner region", notice that equations (4.54) and (5.22) imply

$$
\bar{a} \rightarrow{\frac{e}{\sqrt{x_{\perp}}}}^{-x_{\perp} \sqrt{k^{2}-\left(\omega / c_{\infty}\right)^{2}}} \widetilde{a}\left(\varphi, k, \omega \mid y_{T}\right)
$$

as $x_{T} \rightarrow \infty$ 


$$
\left.\widetilde{\boldsymbol{a}}\left(\varphi, k, \omega \mid \boldsymbol{y}_{\boldsymbol{T}}\right) \equiv \widetilde{\mathcal{G}_{0}}\left(\varphi, k, \omega \mid \boldsymbol{y}_{\boldsymbol{T}}\right)\right|_{y_{\perp}=0}
$$

and use the coordinate transform

$$
y_{1}, y_{2}, y_{3} \rightarrow y_{1}, y_{\perp}, s
$$

together with equations (4.48) and (4.51) to obtain

$$
\begin{gathered}
G_{i i}=\bar{\Gamma}_{i i}=\frac{\partial \bar{g}_{i 4}^{a}}{\partial y_{i}}=-\tilde{a}\left[1+w^{\prime}\left(\alpha_{0} \bar{y}_{\perp}\right)\right], \\
G_{11}=-\frac{2 \alpha_{0} \widetilde{c_{c}^{2}}}{\varepsilon\left(U_{c}^{\prime}\right)^{2}} \tilde{\boldsymbol{a}} w^{\prime}\left(\alpha_{0} \bar{y}_{\perp}\right),
\end{gathered}
$$

where the prime denotes differentiation with respect to the entire argument and

$$
\alpha_{0}^{2} \equiv \frac{i k_{0} U_{c}^{\prime}}{2\left(V_{c}-U_{c} \frac{\partial y_{c}}{\partial Y}\right)}, \quad \text { with } \quad k_{0} \equiv \frac{\omega \cos \theta}{c_{\infty}}
$$

It therefore follows that

$$
2 \operatorname{Re}\left(G_{11} G_{i i}^{*}\right)=|\tilde{\boldsymbol{a}}|^{2}\left[1+w^{\prime}\left(\alpha_{0} \bar{y}_{\perp}\right)\right]^{2} 2 \operatorname{Re} C_{I},
$$

and

$$
\left|G_{11}\right|^{2}=|\tilde{\boldsymbol{a}}|^{2}\left|C_{I}\right|^{2}
$$

where

$$
C_{I} \equiv-\frac{\alpha_{0}^{2} w^{\prime}\left(\alpha_{0} \bar{y}_{\perp}\right)}{2 \varepsilon\left(U_{c}^{\prime}\right)^{2}\left[1+w^{\prime}\left(\alpha_{0} \bar{y}_{\perp}\right)\right]}
$$

Then since equations (4.48) to (4.50) show that 


$$
\begin{gathered}
\frac{1}{2}\left[\frac{\partial \hat{g}_{14}^{a}}{\partial y_{\perp}}-i k \hat{g}_{\perp 4}^{a}-(\gamma-1) U_{c}^{\prime} \hat{g}_{44}^{a}\right]=-\frac{\alpha_{0}}{i k_{0} \sqrt{\varepsilon}\left(U_{c}^{\prime}\right)^{2}}\left[\frac{\partial}{\partial s}\left(\frac{1}{h_{\perp}} \frac{\partial}{\partial s} h_{\perp} \frac{\partial \widetilde{c_{c}^{2}} \tilde{\boldsymbol{a}}}{\partial s}+k^{2} \widetilde{c_{c}^{2}} \tilde{\boldsymbol{a}}\right) w\left(\alpha_{0} \bar{y}_{\perp}\right)\right. \\
\left.\quad+\frac{\tilde{\boldsymbol{a}} \widetilde{c_{c}^{2}}}{\varepsilon} \alpha_{0}^{2} w^{\prime \prime}\left(\alpha_{0} \bar{y}_{\perp}\right)\right]+\frac{\gamma-1}{2} \frac{\tilde{\boldsymbol{a}} U_{c}^{\prime}}{k_{0} \alpha_{0} \sqrt{\varepsilon}} w\left(\alpha_{0} \bar{y}_{\perp}\right) \\
=\frac{-\alpha_{0}^{3} \widetilde{c_{c}^{2}} \tilde{\boldsymbol{a}}}{i k_{0} \varepsilon^{3 / 2}\left(U_{c}^{\prime}\right)^{2}} w^{\prime \prime}\left(\alpha_{0} \bar{y}_{\perp}\right)+\frac{\gamma-1}{2} \frac{U_{c}^{\prime}}{k_{0} \alpha_{0} \sqrt{\varepsilon}} \tilde{\boldsymbol{a}} w\left(\alpha_{0} \bar{y}_{\perp}\right)+O\left(\varepsilon^{-1 / 2}\right) \\
\frac{1}{2}\left(\frac{\partial \hat{g}_{14}^{a}}{\partial s}-i k \hat{g}_{t 4}^{a}\right)=-\frac{\alpha_{0}^{2}}{i k_{0} \varepsilon\left(U_{c}^{\prime}\right)^{2}} \frac{\partial \widetilde{c_{c}^{2}} \tilde{\boldsymbol{a}}}{\partial s} w^{\prime}\left(\alpha_{0} \bar{y}_{\perp}\right),
\end{gathered}
$$

it follows from the coordinate transform (C.7) that

$$
\left|G_{12}\right|^{2}+\left|G_{13}\right|^{2}=\frac{1}{\varepsilon^{3}}\left|\frac{\alpha_{0}^{3} \widetilde{c_{c}^{2}} \tilde{\boldsymbol{a}}}{k_{0}\left(U_{c}^{\prime}\right)^{2}}\right|^{2}\left|w^{\prime \prime}\left(\alpha_{0} \bar{y}_{\perp}\right)\right|^{2}+o\left(\varepsilon^{-3}\right) .
$$

to the lowest order of approximation. And similarly that

$$
\left|G_{23}\right|^{2}-\operatorname{Re} \Gamma_{22} \Gamma_{33}^{*}=\frac{1}{\varepsilon^{3}}\left|\frac{\alpha_{0}^{3} \widetilde{c_{c}^{2}}}{k_{0}^{2}\left(U_{c}^{\prime}\right)^{2}} \frac{\partial \tilde{\boldsymbol{a}}}{\partial s} w^{\prime \prime}\left(\alpha_{0} \bar{y}_{\perp}\right)\right|^{2}+o\left(\varepsilon^{-3}\right) .
$$

Using the product rule (see discussion at the end of section 4), to combine equations (C.1) to (C.17) and retaining only the lowest order approximation within the critical layer, we obtain the uniformly valid approximations to the sums and products

$$
\begin{gathered}
G_{i i}\left(\boldsymbol{x} \mid \boldsymbol{y}_{T}\right)=-\widetilde{\mathcal{G}_{0}}\left(\varphi, \frac{\omega}{c_{\infty}} \cos \theta, \omega \mid \boldsymbol{y}_{T}\right)\left[1+w^{\prime}\left(z_{0}\right)\right] \\
2 \operatorname{Re}_{11} G_{i i}^{*}=2 \operatorname{Re}_{c}\left|\widetilde{\mathcal{G}_{0}}\left(\varphi, \frac{\omega}{c_{\infty}} \cos \theta, \omega \mid \mathbf{y}_{T}\right)\left[1+w^{\prime}\left(z_{0}\right)\right]\right|^{2} \\
\left|G_{11}\right|^{2}=\left|\widetilde{\mathcal{G}_{0}}\left(\varphi, \frac{\omega}{c_{\infty}} \cos \theta, \omega \mid \mathbf{y}_{T}\right)\left[1+w^{\prime}\left(z_{0}\right)\right]\right|^{2}\left|C_{c}\right|^{2}
\end{gathered}
$$




$$
\begin{aligned}
\left|G_{12}\right|^{2}+\left|G_{13}\right|^{2}=\frac{1}{\omega^{2}} \mid \frac{\sqrt{\widetilde{c^{2}}} \cos \theta}{c_{\infty}\left[M\left(\mathbf{y}_{T}\right) \cos \theta-1\right]} \nabla_{T} \frac{\widetilde{\mathcal{G}_{0}} \sqrt{\widetilde{c^{2}}}}{(1-M \cos \theta)} \\
\quad-\left.\frac{(\gamma-1) \widetilde{\mathcal{G}_{0}} c_{\infty}}{2(1-M \cos \theta)} \nabla_{T} M\right|^{2}\left|z_{0}^{3} w^{\prime \prime}\left(z_{0}\right)\right|^{2}+o(1)
\end{aligned}
$$

and

$$
\left|G_{23}\right|^{2}-\operatorname{Re} \Gamma_{22} \Gamma_{33}^{*}=\left(\left|\left(G_{23}\right)_{\text {out }}\right|^{2}-\operatorname{Re}\left(\Gamma_{22}\right)_{\text {out }}\left(\Gamma_{33}^{*}\right)_{\text {out }}\right)\left|z_{0}^{3} w^{\prime \prime}\left(z_{0}\right)\right|^{2}+o(1)
$$

of the dominant $G_{i j}$ components, where $z_{0}$ and $C_{c}$ are given by (6.15) and (6.16).

\section{Appendix D Isotropic Source Model}

Inserting equations(6.11) and (6.12) into(5.31) yields

$$
\begin{aligned}
& I_{\omega}(\boldsymbol{x} \mid \boldsymbol{y}) \rightarrow\left(\frac{2 \pi}{x}\right)^{2} \frac{2 \pi \omega}{c_{\infty}} \sin \theta\left\{| G _ { i i } ( \boldsymbol { x } | \boldsymbol { y } _ { T } ) | ^ { 2 } \left[\tilde{\phi}_{1}\left(\boldsymbol{y} ; \frac{\omega}{\boldsymbol{c}_{\infty}} \cos \theta, \omega\left(1-M_{c} \cos \theta\right)\right)\right.\right. \\
& \left.+G_{i j}\left(\boldsymbol{x} \mid \boldsymbol{y}_{T}\right) G_{i j}^{*}\left(\boldsymbol{x} \mid \boldsymbol{y}_{T}\right) 2 \tilde{\phi}_{2}\left(\boldsymbol{y} ; \frac{\omega}{\boldsymbol{c}_{\infty}} \cos \theta, \omega\left(1-M_{c} \cos \theta\right)\right)\right\} \\
& =\left(\frac{2 \pi}{x}\right)^{2} \frac{2 \pi \omega}{c_{\infty}} \sin \theta\left\{\left|G_{i i}\left(\boldsymbol{x} \mid \boldsymbol{y}_{T}\right)\right|^{2} \Phi^{*}{ }_{1111}\left(\boldsymbol{y} ; \frac{\omega}{c_{\infty}} \cos \theta, \omega\left(1-M_{c} \cos \theta\right)\right)\right. \\
& \left.+2\left[G_{i j}\left(\boldsymbol{x} \mid \boldsymbol{y}_{T}\right) G_{i j}^{*}\left(\boldsymbol{x} \mid \boldsymbol{y}_{T}\right)-\left|G_{i i}\left(\boldsymbol{x} \mid \boldsymbol{y}_{T}\right)\right|^{2}\right] \Phi^{*}{ }_{1212}\left(\boldsymbol{y} ; \frac{\omega}{c_{\infty}} \cos \theta, \omega\left(1-M_{c} \cos \theta\right)\right)\right\}
\end{aligned}
$$

$$
\text { as } x \rightarrow \infty
$$

where $G_{i j}$ is defined by(5.32). Then since

$$
\begin{aligned}
& \left|G_{i i}\left(\boldsymbol{x} \mid \boldsymbol{y}_{T}\right)\right|^{2}= \\
& \quad\left|G_{11}\left(\boldsymbol{x} \mid \boldsymbol{y}_{T}\right)\right|^{2}+\left|G_{22}\left(\boldsymbol{x} \mid \boldsymbol{y}_{T}\right)\right|^{2}+\left|G_{33}\left(\boldsymbol{x} \mid \boldsymbol{y}_{T}\right)\right|^{2}+2 \boldsymbol{R e}\left(G_{22} G_{11}^{*}+G_{33} G_{11}^{*}+G_{22} G_{33}^{*}\right)
\end{aligned}
$$

and 


$$
\begin{aligned}
& G_{i j}\left(\boldsymbol{x} \mid \boldsymbol{y}_{T}\right) G_{i j}^{*}\left(\boldsymbol{x} \mid \boldsymbol{y}_{T}\right) \\
& =\left|G_{11}\left(\boldsymbol{x} \mid \boldsymbol{y}_{T}\right)\right|^{2}+\left|G_{22}\left(\boldsymbol{x} \mid \boldsymbol{y}_{T}\right)\right|^{2}+\left|G_{33}\left(\boldsymbol{x} \mid \boldsymbol{y}_{T}\right)\right|^{2}+2\left(\left|G_{21}\right|^{2}+\left|G_{31}\right|^{2}+\left|G_{23}\right|^{2}\right)
\end{aligned}
$$

And, in view of (C.18) to(C.20), it follows that

$$
\begin{aligned}
& G_{i j}\left(\boldsymbol{x} \mid \boldsymbol{y}_{T}\right) G_{i j}^{*}\left(\boldsymbol{x} \mid \boldsymbol{y}_{T}\right)-\left|G_{i i}\left(\boldsymbol{x} \mid \boldsymbol{y}_{T}\right)\right|^{2}=2\left[\left|G_{21}\right|^{2}+\left|G_{31}\right|^{2}+\left|G_{23}\right|^{2}-\operatorname{Re}\left(G_{22} G_{11}^{*}+G_{33} G_{11}^{*}+G_{22} G_{33}^{*}\right)\right] \\
& =2\left[\left|G_{21}\right|^{2}+\left|G_{31}\right|^{2}+\left|G_{23}\right|^{2}+\left|G_{11}\right|^{2}-\operatorname{Re}\left(G_{i i} G_{11}^{*}+G_{22} G_{33}^{*}\right)\right] \\
& =2\left[\left|G_{21}\right|^{2}+\left|G_{31}\right|^{2}+\left|G_{23}\right|^{2}-\operatorname{Re}_{22} G_{33}^{*}+\operatorname{Re}_{c}\left(C_{c}^{*}-1\right)\left[1+w^{\prime}\left(z_{0}\right)\right]^{2}\left|\widetilde{\mathcal{G}_{0}}\left(\varphi, \frac{\omega}{c_{\infty}} \cos \theta, \omega \mid \boldsymbol{y}_{T}\right)\right|^{2}\right]
\end{aligned}
$$

and, therefore that

$$
\begin{aligned}
& I_{\omega}(\boldsymbol{x} \mid \boldsymbol{y}) \rightarrow\left(\frac{2 \pi}{x}\right)^{2} \frac{2 \pi \omega}{c_{\infty}} \sin \theta\left\{\left[\Phi{ }_{1111}\left(\boldsymbol{y} ; \frac{\omega}{c_{\infty}} \cos \theta, \frac{\omega}{c_{\infty}} \nabla_{\boldsymbol{y}_{T}} S, \omega\left(1-M_{c} \cos \theta\right)\right)\right.\right. \\
& \left.+4 \operatorname{Re}_{c}\left(C_{c}^{*}-1\right) \Phi^{*}{ }_{1212}\left(\boldsymbol{y} ; \frac{\omega}{c_{\infty}} \cos \theta, \frac{\omega}{c_{\infty}} \nabla_{\boldsymbol{y}_{T}} S, \omega\left(1-M_{c} \cos \theta\right)\right)\right]\left|\widetilde{\mathcal{G}_{0}}\left(\varphi, \frac{\omega}{c_{\infty}} \cos \theta, \omega \mid \boldsymbol{y}_{T}\right)\left[1+w^{\prime}\left(z_{0}\right)\right]\right|^{2} \\
& \left.+4\left[\left|G_{21}\right|^{2}+\left|G_{31}\right|^{2}+\left(\left|G_{23}\right|^{2}-\operatorname{Re}_{22} G_{33}^{*}\right)\right] \Phi_{1212}^{*}\left(\boldsymbol{y} ; \frac{\omega}{c_{\infty}} \cos \theta, \frac{\omega}{c_{\infty}} \nabla_{\boldsymbol{y}_{T}} S, \omega\left(1-M_{c} \cos \theta\right)\right)\right\}
\end{aligned}
$$

where $C_{c}$ is given by(6.16). And, it now follows from (C.21) and(C.22) that equation (6.14) holds.

\section{Appendix E Axisymmetric source Model}

Goldstein \& Rosenbaum (1973 a) show that

$$
H_{i j} G_{k l}^{*} I_{i k j l}=H_{i i} G_{k k}^{*} I_{1212}-\left(H_{22} G_{33}^{*}+H_{33} G_{22}^{*}\right)\left(I_{1212}-I_{2323}\right)+H_{i j} G_{i j}^{*} Q_{i j}
$$

with $I_{i j k l}$ defined by equation (6.23) $Q_{11} \equiv I_{1111}-I_{1212}, \quad Q_{12}=Q_{13} \equiv I_{1212}+I_{1122}$, 
$Q_{22}=Q_{33}=I_{2222}-I_{1212}, Q_{23}=I_{2222}-I_{2323}$ for any $2^{\text {nd }}$ order symmetric tensors $G_{i j}$ and $H_{i j}$ and any axisymmetric tensorial function $R_{i j}^{M}(\xi)$ of $\xi$. It, therefore, follows from equations (D.2) and(D.3) that

$$
\begin{aligned}
& G_{i j} G_{k l}^{*} I_{i k j l}=\left|G_{11}\right|^{2} I_{1111}+\left(\left|G_{22}\right|^{2}+\left|G_{33}\right|^{2}-2\left|G_{23}\right|^{2}\right) I_{2222}+2 \operatorname{Re}\left(G_{22} G_{33}^{*}-\left|G_{23}\right|^{2}\right) I_{2323} \\
& +2\left(\left|G_{12}\right|^{2}+\left|G_{13}\right|^{2}\right) I_{1122}+2\left[\left|G_{12}\right|^{2}+\left|G_{13}\right|^{2}+\operatorname{Re} G_{11}\left(G_{22}^{*}+G_{33}^{*}\right)\right] I_{1212} \\
& =\left|G_{11}\right|^{2}\left(I_{1111}-2 I_{1212}\right)+2 \operatorname{Re} G_{11} G_{i i}^{*} I_{1212}+2\left(\left|G_{12}\right|^{2}+\left|G_{13}\right|^{2}\right)\left(I_{1122}+I_{1212}\right) \\
& +\left(\left|G_{22}\right|^{2}+\left|G_{33}\right|^{2}+2\left|G_{23}\right|^{2}\right) I_{2222}+2 \operatorname{Re}\left(G_{22} G_{33}^{*}-\left|G_{23}\right|^{2}\right) I_{2323}+\left(\left|G_{22}\right|^{2}+\left|G_{33}\right|^{2}-2\left|G_{23}\right|^{2}\right) I_{2222} \\
& =\left|G_{11}\right|^{2}\left(I_{1111}-2 I_{1212}\right)+2 \operatorname{Re} G_{11} G_{i i}^{*} I_{1212}+\left(\left|G_{i i}\right|^{2}-2 \operatorname{Re} G_{11} G_{i i}^{*}+\left|G_{11}\right|^{2}\right) I_{2222} \\
& +2 \operatorname{Re}\left(G_{22} G_{33}^{*}-\left|G_{23}\right|^{2}\right)\left(I_{2323}-I_{2222}\right)+2\left(\left|G_{12}\right|^{2}+\left|G_{13}\right|^{2}\right)\left(I_{1122}+I_{1212}\right)
\end{aligned}
$$

It, therefore follows from (C.18) to (C.22) and (6.16) that equation (6.24)holds.

Taking $H_{i j}=\delta_{i j}$ shows that

$$
G_{k l}^{*} I_{i k i l}=G_{k k}^{*}\left(I_{1212}+I_{2222}+I_{2323}\right)+G_{11}^{*}\left(I_{1111}+I_{1212}-I_{2222}-I_{2323}\right)
$$

And taking $H_{i j}=G_{i j}^{*}=\delta_{i j}$ shows that

$$
I_{i j i j}=4 I_{1212}+2 I_{2222}+2 I_{2323}+I_{1111}
$$

\section{Appendix F Details of Spectral Component Model}

Integrating equation(6.34) by parts shows that

$$
\Psi_{i j k l}=\frac{l_{1} l_{T}^{2}}{\lambda} \iint_{-\infty}^{\infty} \int_{-\infty}^{\infty} \sum_{m, l=0}^{\infty} a_{m, l}\left[(-1)^{m+l} D_{k_{1}}^{m} D_{\omega}^{l} e^{i\left(\xi_{1} k_{1}+\xi_{T} \cdot \boldsymbol{k}_{T}-\omega \tau\right)}\right] e^{-X+\gamma} d \tilde{\xi}_{T} d \tilde{\tau} d \tilde{\xi}_{1}
$$


where $D_{k_{1}}, D_{\omega}$, and $D_{k_{T}}$ are defined by equations(6.32). Then since (Campbell \& Foster,1942, p111, \#867)

$$
\int_{-\infty}^{\infty} e^{-i \omega \tau-X} d \tilde{\tau}=\frac{2 \sqrt{\tilde{\xi}_{1}^{2}+\beta^{2}} K_{1}\left(\sqrt{\tilde{\xi}_{1}^{2}+\beta^{2}} \sqrt{1+\tilde{\omega}^{2}}\right)}{\sqrt{1+\tilde{\omega}^{2}}}
$$

where $K_{1}$ is a modified Bessel function of the second kind. (Campbell \& Foster, 1942, p125, \#917.8) and equation (6.30) show that equation (F.1) can be written as

$$
\begin{aligned}
& \Psi_{i j k l}\left(k_{1}, \tilde{\boldsymbol{k}}_{T}, \omega\right) \\
& =\frac{l_{1} l_{T}^{2}}{\lambda} \sum_{m, l=0}^{\infty} a_{m, l}(-1)^{m+l} D_{k_{1}}^{m} D_{\omega}^{l} \int\left[\frac{1}{\left(1+R^{2}\right)^{3 / 2}}+\frac{\beta}{\left(1+R^{2}\right)}\right] e^{i \boldsymbol{\xi}_{T} \cdot \boldsymbol{k}_{T}-\beta\left(1+R^{2}\right)^{1 / 2}+\gamma} d \tilde{\boldsymbol{\xi}}_{T} \\
& =\frac{l_{1} l_{T}^{2}}{\lambda} \sum_{m, l=0}^{\infty} a_{m, l}(-1)^{m+l} D_{k_{1}}^{m} D_{\omega}^{l}\left\{\frac{1}{\left(1+R^{2}\right)^{3 / 2}}-\frac{1}{\left(1+R^{2}\right)^{1 / 2} R} \frac{d}{d R}\right\} \int e^{i \boldsymbol{\xi}_{T} \cdot \boldsymbol{k}_{T}-\beta\left(1+R^{2}\right)^{1 / 2}+\gamma} d \tilde{\boldsymbol{\xi}}_{T} \\
& =\frac{l_{1} l_{T}^{2}}{\lambda} \sum_{m, l=0}^{\infty} a_{m, l}(-1)^{m+l} D_{k_{1}}^{m} D_{\omega}^{l} \frac{1}{R} \frac{d}{d R} \frac{\int e^{i \xi_{T} \cdot \boldsymbol{k}_{T}-\beta\left(1+R^{2}\right)^{1 / 2}+\gamma} d \tilde{\boldsymbol{\xi}}_{T}}{\left(1+R^{2}\right)^{1 / 2}}
\end{aligned}
$$

which can now be rewritten as equation (6.35) .

\section{Appendix G Axisymmetric Jet}

When expressed in polar coordinates, the coefficients in equations(6.14) and (6.27) become

$$
\left|C \nabla_{T} \frac{\widetilde{\mathcal{G}_{0}} \sqrt{\widetilde{c^{2}}}}{(1-M \cos \theta)}-\frac{(\gamma-1) \widetilde{\mathcal{G}_{0}} c_{\infty}}{2(1-M \cos \theta)} \nabla_{T} M\right|^{2}
$$




$$
=\left|C \frac{\partial}{\partial r_{\perp}} \frac{\widetilde{\mathcal{G}_{0}} \sqrt{\widetilde{c^{2}}}}{(1-M \cos \theta)}-\frac{(\gamma-1) \widetilde{\mathcal{G}_{0}} c_{\infty}}{2(1-M \cos \theta)} \frac{\partial}{\partial r_{\perp}} M\right|^{2}+\frac{\widetilde{c^{2}} C^{2}}{(1-M \cos \theta)^{2} r_{\perp}^{2}}\left|\frac{\partial \widetilde{\mathcal{G}_{0}}}{\partial \varphi^{\prime}}\right|^{2}
$$

And

$$
\begin{aligned}
\left|\left(G_{23}\right)_{\text {out }}\right|^{2}-\mathcal{R e}_{e}\left(\Gamma_{22}\right)_{\text {out }}\left(\Gamma_{33}^{*}\right)_{\text {out }}=\frac{\widetilde{c^{2}}}{(1-M \cos \theta)^{2} \omega^{4}}\left[\left|\frac{\partial^{2}}{\partial r_{\perp} \partial \varphi^{\prime}}\left(\frac{\widetilde{\mathcal{G}_{0}} \sqrt{c^{2}}}{r_{\perp}(1-M \cos \theta)}\right)\right|^{2}\right. \\
\left.-\mathbb{R e}_{e}\left(\frac{\partial}{\partial r_{\perp}} \frac{\widetilde{\mathcal{G}_{0}}{\widetilde{r_{\perp}}}^{\widetilde{c^{2}}}}{(1-M \cos \theta)^{2}}\right)\left(\frac{1}{r_{\perp}^{2}} \widetilde{\mathcal{G}}_{o \varphi^{\prime} \varphi^{\prime}}^{*}+\frac{1}{r_{\perp}} \widetilde{\mathcal{G}}_{o r_{\perp}}^{*}\right)\right]
\end{aligned}
$$

where $C$ is defined by (C.4). Since

$$
\begin{aligned}
& \operatorname{Re}\left(\frac{\partial}{\partial r_{\perp}} \frac{\widetilde{\mathcal{G}_{0}}{\widetilde{r_{\perp}}}^{c^{2}}}{(1-M \cos \theta)^{2}}\right)\left(\frac{1}{r_{\perp}^{2}} \widetilde{\mathcal{G}}_{O \varphi^{\prime} \varphi^{\prime}}^{*}+\frac{1}{r_{\perp}} \widetilde{\mathcal{G}}_{r_{\perp}}^{*}\right)=-\operatorname{Re}\left\{\frac{\widetilde{c^{2}}}{(1-M \cos \theta)^{2}}\left(\frac{1}{r_{\perp}^{2}} \widetilde{\mathcal{G}}_{o \varphi^{\prime} \varphi^{\prime}}+\frac{1}{r_{\perp}} \widetilde{\mathcal{G}}_{{ }_{r_{\perp}}}\right)\right. \\
& \left.+\omega^{2}\left(1-C^{2}\right) \widetilde{\mathcal{G}_{0}}\right\}\left(\frac{1}{r_{\perp}^{2}} \widetilde{\mathcal{G}}_{O \varphi^{\prime} \varphi^{\prime}}^{*}+\frac{1}{r_{\perp}} \widetilde{\mathcal{G}}_{O r_{\perp}}^{*}\right)=-\mathcal{R e}_{e}\left[\frac{\widetilde{c^{2}}}{(1-M \cos \theta)^{2}} \mid \frac{1}{r_{\perp}^{2}} \widetilde{\mathcal{G}}_{O \varphi^{\prime} \varphi^{\prime}}+\frac{1}{r_{\perp}} \widetilde{\mathcal{G}}_{r_{\perp}}\right. \\
& \left.+\left.\frac{\omega^{2}\left(1-C^{2}\right) \widetilde{\mathcal{G}_{0}}(1-M \cos \theta)^{2}}{2 \overline{c^{2}}}\right|^{2}\right]-\frac{\omega^{4}\left(1-C^{2}\right)^{2}\left|\widetilde{\mathcal{G}_{0}}\right|^{2}(1-M \cos \theta)^{2}}{4 c^{2}}
\end{aligned}
$$

equation (G.2) becomes

$$
\begin{aligned}
& \left|\left(G_{23}\right)_{\text {out }}\right|^{2}-\operatorname{Re}\left(\Gamma_{22}\right)_{\text {out }}\left(\Gamma_{33}^{*}\right)_{\text {out }}=\frac{\widetilde{c^{2}}}{(1-M \cos \theta)^{2} \omega^{4}}\left[\left|\frac{\partial^{2}}{\partial r_{\perp} \partial \varphi^{\prime}}\left(\frac{\widetilde{\mathcal{G}_{0}} \sqrt{\widetilde{c^{2}}}}{r_{\perp}(1-M \cos \theta)}\right)\right|^{2}\right. \\
& +\frac{\widetilde{c^{2}}}{(1-M \cos \theta)^{2}} \mid \frac{1}{r_{\perp}^{2}} \widetilde{\mathcal{G}}_{\text {o }^{\prime} \varphi^{\prime}}+\frac{1}{r_{\perp}} \widetilde{\mathcal{G}}_{r_{\perp}}
\end{aligned}
$$




$$
\left.+\left.\frac{\omega^{2}\left(1-C^{2}\right) \widetilde{\mathcal{G}_{0}}(1-M \cos \theta)^{2}}{2 \widetilde{c^{2}}}\right|^{2}-\frac{\omega^{4}\left(1-C^{2}\right)^{2}\left|\widetilde{\mathcal{G}_{0}}\right|^{2}(1-M \cos \theta)^{2}}{4 \widetilde{c^{2}}}\right]
$$

Integrating these over the circumferential direction and using equation(7.11) shows that

$$
\begin{gathered}
\frac{1}{2 \pi} \int_{0}^{2 \pi}\left|\widetilde{\mathcal{G}_{0}}\left(\varphi^{\prime}, \frac{\omega}{c_{\infty}} \cos \theta, \omega \mid \mathbf{y}_{\perp}\right)\right|^{2} d \varphi^{\prime}=\sum_{n=-\infty}^{\infty}\left|\frac{w_{n}^{(2)}\left(r_{\perp}^{\prime}, \frac{\omega}{c_{\infty}} \cos \theta\right)}{\Delta_{n}}\right|^{2} \\
\frac{1}{2 \pi} \int_{0}^{2 \pi}\left|C \nabla_{T} \frac{\widetilde{\mathcal{G}_{0}} \sqrt{\bar{c}^{2}}}{(1-M \cos \theta)}-\frac{(\gamma-1) \widetilde{\mathcal{G}_{0}} c_{\infty}}{2(1-M \cos \theta)} \nabla_{T} M\right|^{2} d \varphi^{\prime}= \\
\sum_{n=-\infty}^{\infty}\left|C \frac{\partial}{\partial r_{\perp}}\left(\frac{w_{n}^{(2)} \sqrt{\widetilde{c^{2}}}}{\Delta_{n}(1-M \cos \theta)}\right)-\frac{(\gamma-1) \widetilde{\mathcal{G}_{0}} c_{\infty}}{2 \Delta_{n}(1-M \cos \theta)} \frac{\partial}{\partial r_{\perp}} M\right|^{2} \\
+\frac{C^{2} \widetilde{c^{2}}}{r_{\perp}^{2}(1-M \cos \theta)^{2}} \sum_{n=-\infty}^{\infty} n^{2}\left|\frac{w_{n}^{(2)}}{\Delta_{n}}\right|^{2}
\end{gathered}
$$

and

$$
\begin{aligned}
& \left.\frac{1}{2 \pi} \int_{0}^{2 \pi}||\left(G_{23}\right)_{\text {out }}\right|^{2}-\left.\operatorname{Re}\left(\Gamma_{22}\right)_{\text {out }}\left(\Gamma_{33}^{*}\right)_{\text {out }}\right|^{2} d \varphi^{\prime}=-\sum_{n=-\infty}^{\infty} \frac{\left(1-C^{2}\right)^{2}\left|w_{n}^{(2)}\right|^{2}}{4 \Delta_{n}^{2}} \\
& +\sum_{n=-\infty}^{\infty}\left|\frac{\overline{c^{2}}}{\omega^{2}(1-M \cos \theta)^{2}}\left[\frac{1}{r_{\perp}} \frac{\partial}{\partial r_{\perp}}\left(\frac{w_{n}^{(2)}}{\Delta_{n}}\right)-\frac{n^{2} w_{n}^{(2)}}{r_{\perp}^{2} \Delta_{n}}\right]+\frac{\left(1-C^{2}\right) w_{n}^{(2)}}{2 \Delta_{n}}\right|^{2} \\
& +\frac{\overline{c^{2}}}{\omega^{2}(1-M \cos \theta)^{2}} \sum_{n=-\infty}^{\infty} n^{2}\left|\frac{\partial}{\partial r_{\perp}}\left(\frac{w_{n}^{(2)} \sqrt{c^{2}}}{r_{\perp} \omega \Delta_{n}(1-M \cos \theta)}\right)\right|^{2}
\end{aligned}
$$

Inserting these into equations (4.21)and (6.27) shows that (7.12) holds. 


\section{REFERENCES}

Abramowitz, M. \& Stegun, I.A., 1965, Handbook of Mathematical Functions, National Bureau of Standards, Washington

Afsar, M. Z., Dowling, A.P.,\& Karabasov, S. A., 2006, Comparison of jet noise models, $12^{\text {th }}$ AIAA/CEAS Aeroacoustics Conference, Cambridge, Mass.

Afsar, M. Z., Dowling, A.P.,\& Karabasov, S. A., 2007, Jet Noise in the Zone of Silence, $13^{\text {th }}$ AIAA/CEAS Aeroacoustics Rome, Italy

Balsa, T. F., 1977, The acoustic field of sources in shear flow with application to jet noise: convective amplification, Journal of Fluid Mechanics, vol.70 , pp. 3347.

Batchelor, G.K., 1953, Theory of Homogeneous Turbulence," Cambridge University Press,

Bridges, J. and Podboy, G. G., 1999, Measurements of Two-Point Velocity Correlations in a Round Jet with Application to Jet Noise , AIAA Paper \#19991966,

Campbell, G.A. and Foster, R. M.,1942, Fourier Integrals for Practical Applications, The American Telephone and Telegraph Company

Crighton, D.G.,1975, Basic Principles of Aerodynamic Noise Generation, Progress in Aerospace Sciences, Vol. 16, pp. 31-96

Crighton, D.G.,1979, Why Do the Acoustics and the Dynamics of a Hypothetical Mean Flow Bear on the Issue of Sound generated by Turbulence?, Mechanics of Sound Generation in Flows, Springer-Verlag, Heidelberg, pp.1-11.

Dowling, A.P. \& Ffowcs Williams, J. E, 1983, Sound and Sources of Sound, Ellis Horwood Limited, Chichester

Favre, A.,1969, Statistical Equations of Turbulent Gases", Problems of Hydrodynamics and Continuum Mechanics, SIAM, Philadelphia, pp.1-7.

Ffowcs Williams, J.E., 1963, The Noise from Turbulence Convected at High Speed, Phil. Trans. Roy. Soc., A 225, pp. 469-503

Ffowcs Williams,J.E.,1969, Hydrodynamic Noise" Annual Review of Fluid Mechanics vol.1, pp. 197-222

Freund, J.B., 2002, Turbulent Jet Noise: Shear Noise, Self-Noise, and Other Contributions, AIAA Paper 2002-2423. 
Goldstein, M.E., 2002, A unified approach to some recent developments in jet noise theory International Journal of Aeroacoustics, vol. 1,no. 1, pp. 1-16.

Goldstein, M.E., 2003, A Generalized Acoustic Analogy, Journal of Fluid Mechanics, vol. 488, pp. 315-333.

Goldstein, M.E., 2004, The $90^{\circ}$ Acoustic Spectrum of a High Speed Air Jet, AIAA Journal, Vol.42, No.11, pp. 96-102.

Goldstein, M.E.,1975, The Low Frequency Sound from Multipole Sources in Axisymmetric Shear Flows with Application to Jet Noise, Journal of Fluid Mechanics, vol.70, No.3, pp.595-604.

Goldstein, M.E. and Rosenbaum, B.M., 1973 a, Emission of Sound from Turbulence Convected by a Parallel Flow in the Presence of Solid Boundaries, NASA TN D-7118.

Goldstein, M.E. and Rosenbaum, B. M., 1973 b, Effect of Anisotropic Turbulence on Aerodynamic Noise, Journal of the Acoustical Society of America, vol. 54, no. 3, pp. 630-645.

Goldstein, M.E. and Leib, S. J., 2005, The role of instability waves in predicting jet noise Journal of Fluid Mechanics, Volume 525, pp 37-72

Goldstein, M.E., 2006, Hybrid Reynolds-Averaged Navier-Stokes/ Large Eddy Simulation Approach for Predicting Jet Noise, AIAA Journal, vol. 44,No. 12, pp3136-3142

Gradshteyn, I. S. and Ryzhik, I. M., 1965, Table of Integrals, Series, and Products, Academic Press, New York

Harper-Bourne, M., 2003, Jet Noise Turbulence Measurements, AIAA Paper 2003-3214

Kerschen, E.J.,1983, Constraints on the Invariant Function of Axisymmetric Turbulence, AIAA Journal, vol. 21, no. 7, , pp. 978-985.

Khavaran, A., Bridges, J., and Freund, J.B., 2002, A Parametric Study of FineScale Turbulence Mixing Noise, NASA/TM-2002-211696. 
Khavaran, A. and Bridges, J., 2004, Modeling of Turbulence Generated Noise in Jets, AIAA paper \# 2004-2983, Presented at the $10^{\text {th }}$ AIAA/CEAS Aeroacoustics Conference \& Exhibit, Manchester, U. K.

Khavaran, A and Kenzakowski, D. C., 2007, Progress Toward Improving Jet Noise Predictions in Hot Jets, AIAA paper \# 2007-0012

Khavaran,A., Bridges, J. \& Georgiadis, N., 2005, Prediction of turbulence generated in unheated jets, NASA/TM-2005-213827

Lighthill, M.J.,1952, On Sound Generated Aerodynamically: I. General Theory, Proceedings R. Society Lond., A 211, pp. 564-587

Lilley, G.M., 1972, Generation of Sound in a Mixing Region," Lockheed Aircraft Co. $4^{\text {th }}$ Monthly progress report on contract F-33615-71-C-1663, Marietta

Lilley, G.M., 1974, "On the Noise from Jets," Noise Mechanism, AGARD-CP131

Lilley, G.M., 1996, The Radiated Noise from Isotropic Turbulence with Applications to the Theory of Jet Noise, Journal of Sound Vibration, Vol. 190, pp. 463-476.

Morfey, C.L., Szewczyk, V.M., and Tester, B.J., 1978, New Scaling Laws for Hot and Cold Jet Mixing Noise Based on a Geometric Acoustics Model, Journal of Sound and Vibration, vol. 61, no. 2, pp.255-292.

Morse, P.M. and Feshbach, H.,1953, Methods of Theoretical Physics, McGrawHill,.

Musifar, R. E., 1992, On the solution of Lilley's equation, Proceedings of the $14^{\text {th }}$ Congress on Acoustics-ICA, Beijing, vol4. paper K2-7.

Musifar, R. E., 1993 "A note on the description of jet noise source terms", Proc. Institute of Acoustics (UK) Vol. 15, part 3(4), 901-909.

Musifar, R. E.,2006, "Relating the spectra of hot and cold jets", Proc. 13 ICSV, Vienna.

Nelson, C. C. and Power, G.D., 2001, "CHSSI Project CFD-7:The NPARC Alliance Flow Simulation System," AIAA Paper 2001-0594.

Phillips, O.M. ,1960, On the Generation of Sound by Supersonic Turbulent Shear Layers, J. Fluid Mech., vol. 9, pp. 1-28.

Pope, S. B.,2000, Turbulent flows, Cambridge University Press 
Ribner, H. S., 1969, Quadrupole Correlations Governing the Pattern of Jet Noise, J. Fluid Mach. Vol. 38, pp. 1-8

Van Dyke, M., 1975, Perturbation Methods in Fluid Mechanics, the Parabolic Press, Stanford CA

Wundrow, D.W. \& Goldstein, M. E. 1994, Nonlinear Instability of a Uni-Directional Transversely Sheared Mean Flow, NASA Technical Memorandum 106779

Viswanathan, K.,2007, Improved Method for Prediction of Noise from Single Jets, AIAA Journal, vol. 45, No. 1, pp. 151-161

Watson, G. N., 1966, A Treatise on the Theory of Bessel Functions, Cambridge University Press

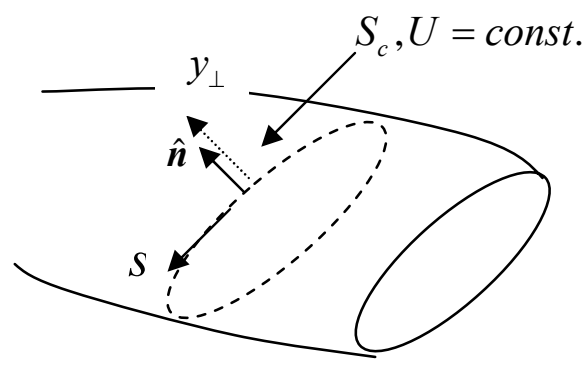

Figure 1 Critical layer surface 


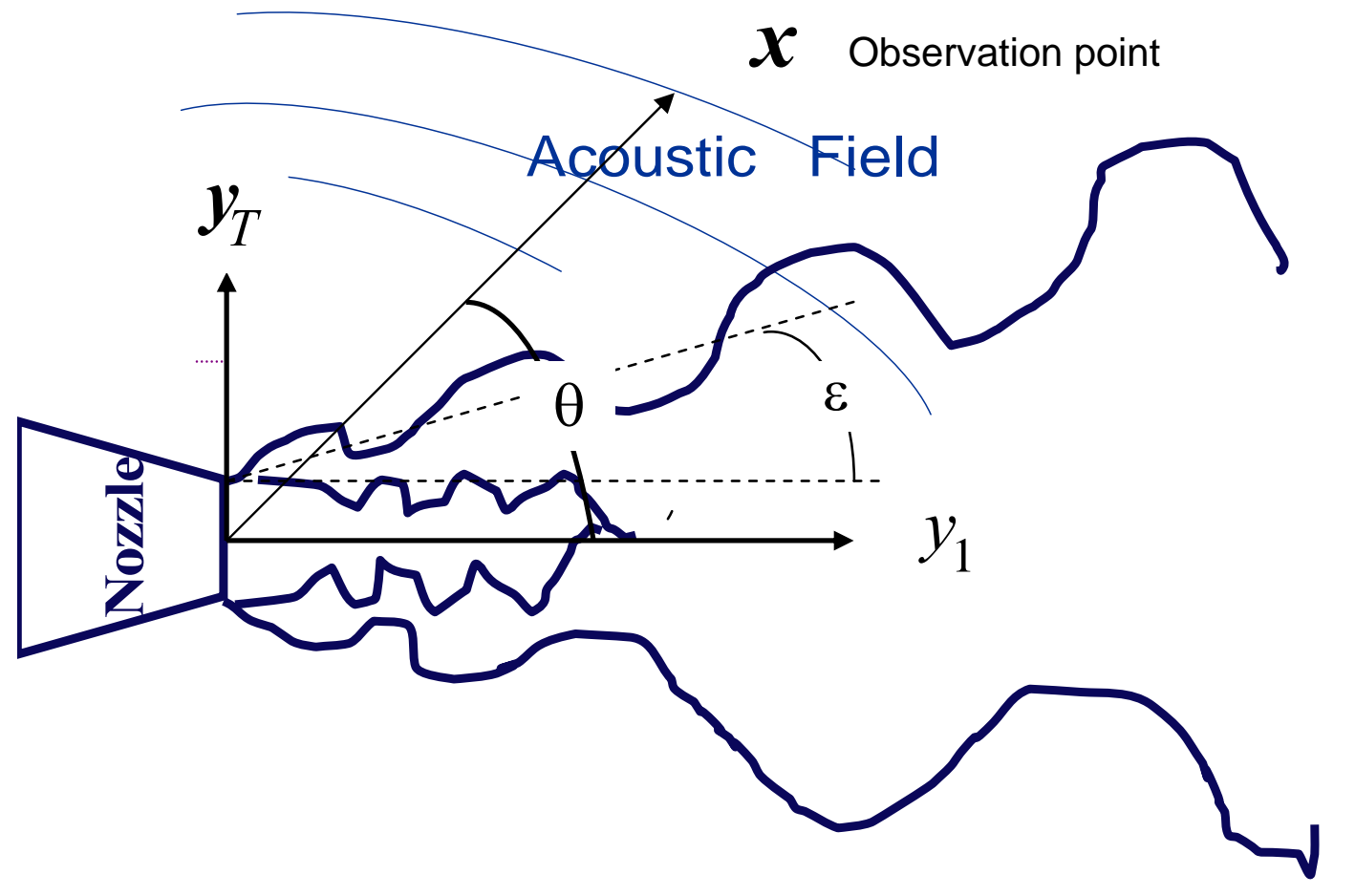

Figure 2 Flow Configuration

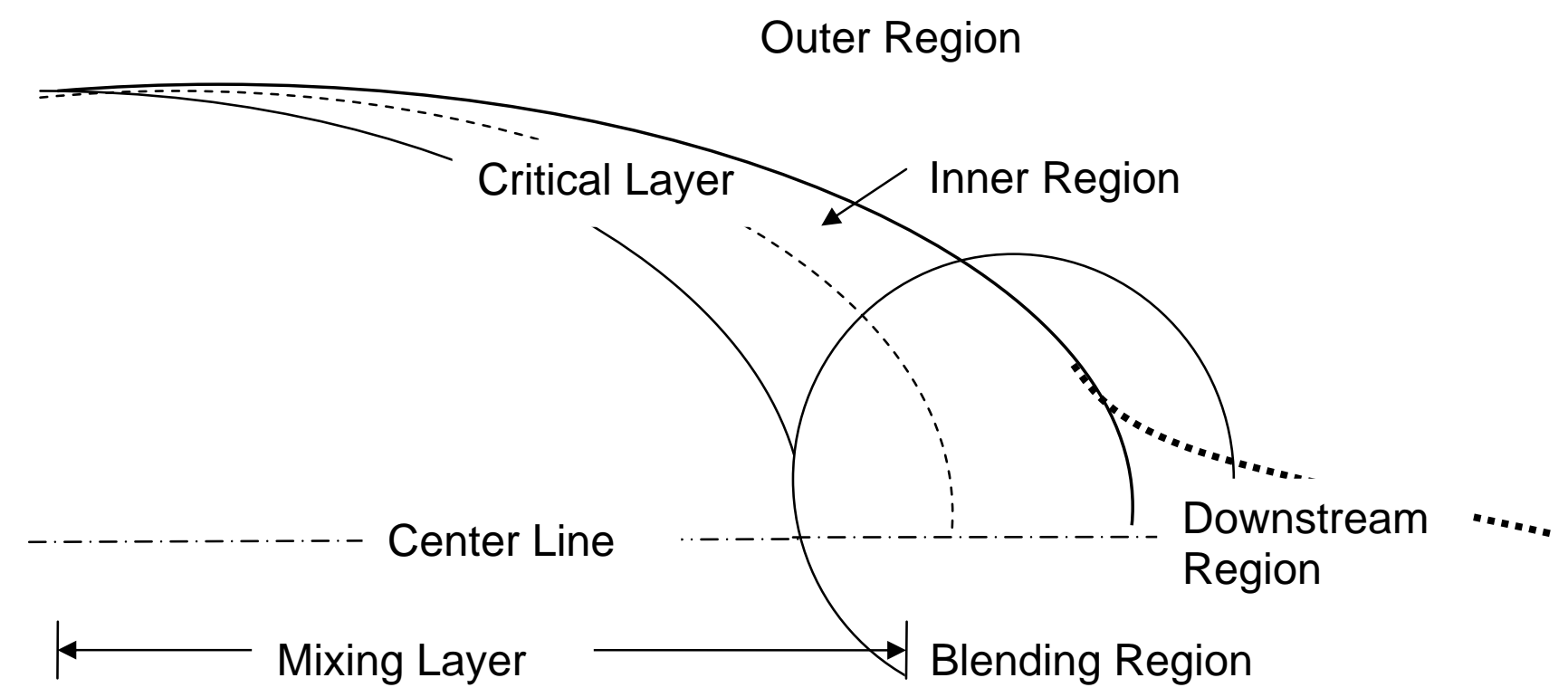

Figure 3 Critical Layer Structure in Supersonic Jet 


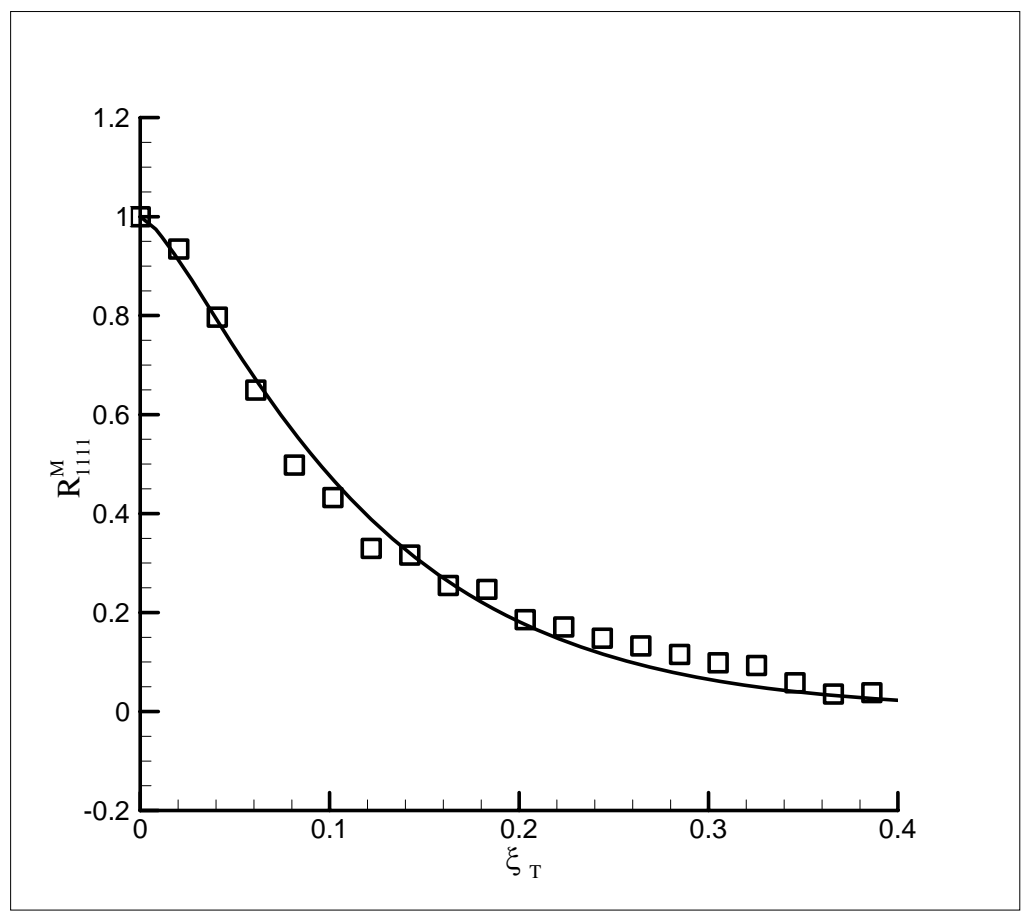

Figure 4 Comparison of the functional form (6.32) with experimental data.

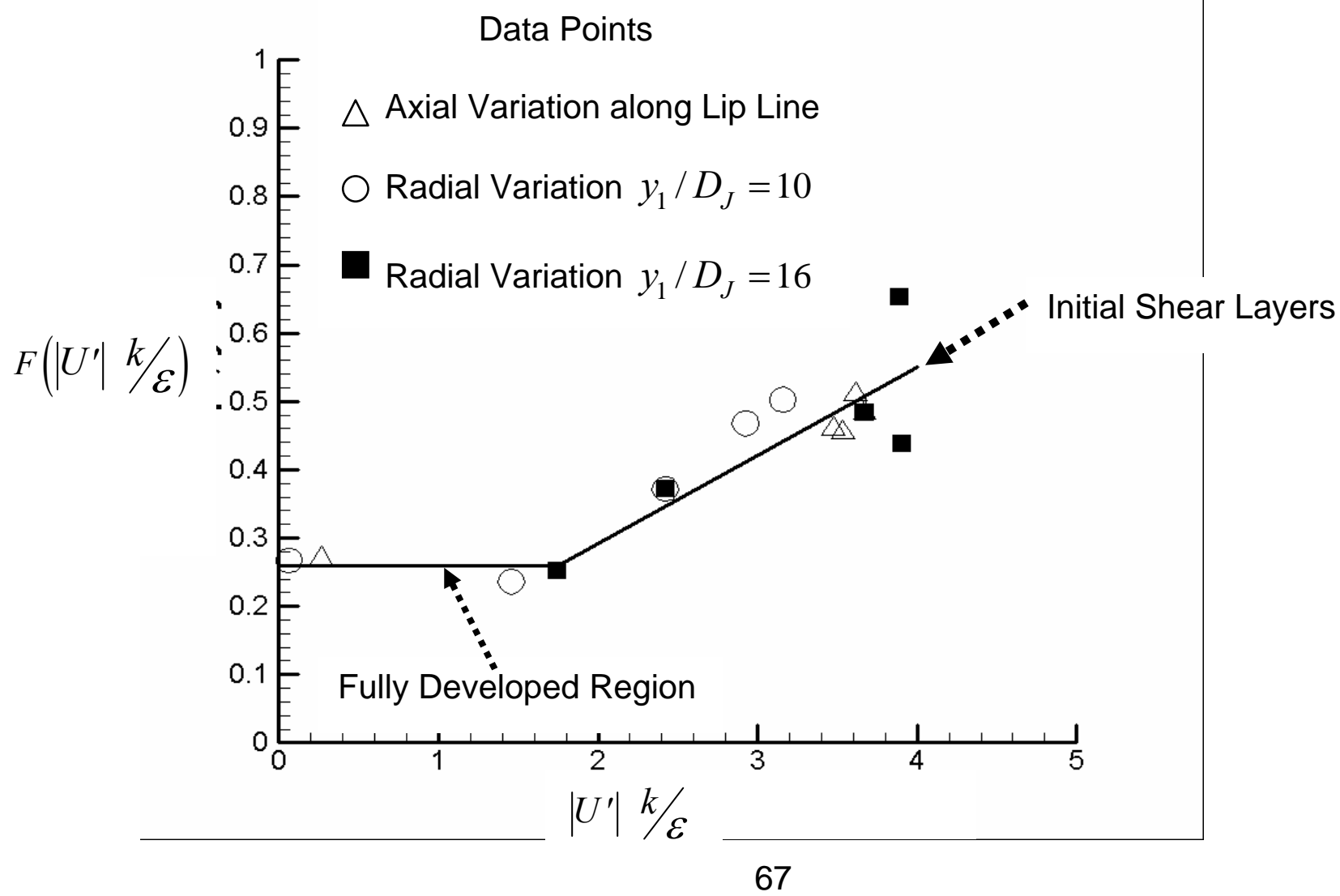


Figure 5 Variation of $C_{\tau} / C_{\tau}^{(0)}$ with time scale parameter $\left|U^{\prime}\right| k / \varepsilon$

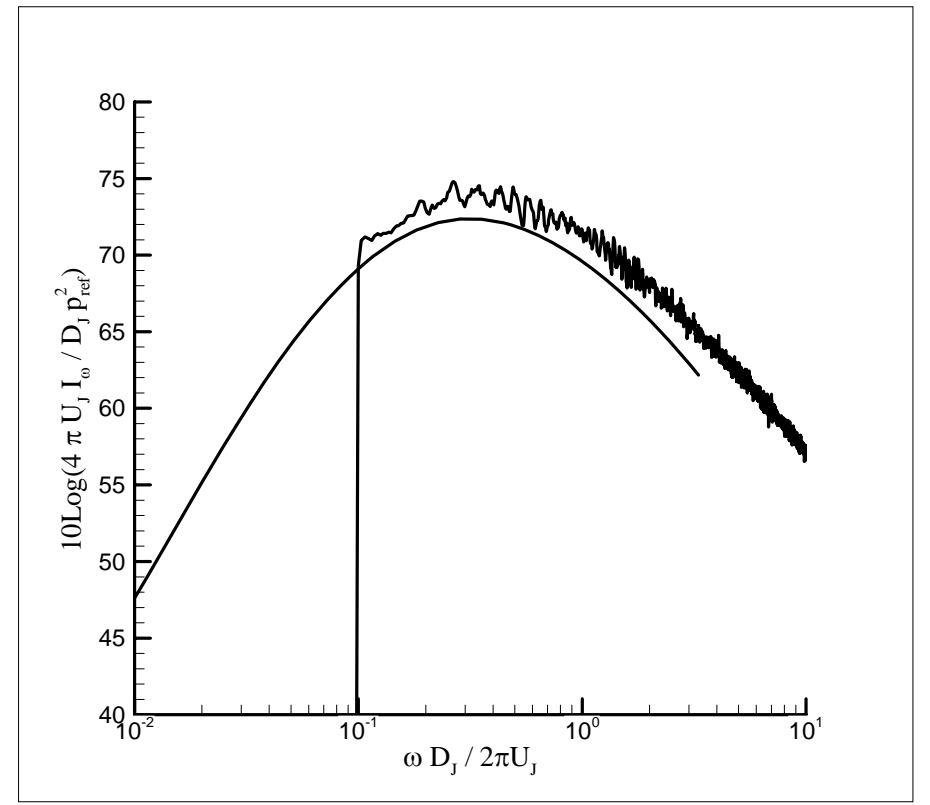

Part a) $M_{J}=0.5, \theta=90^{\circ}$

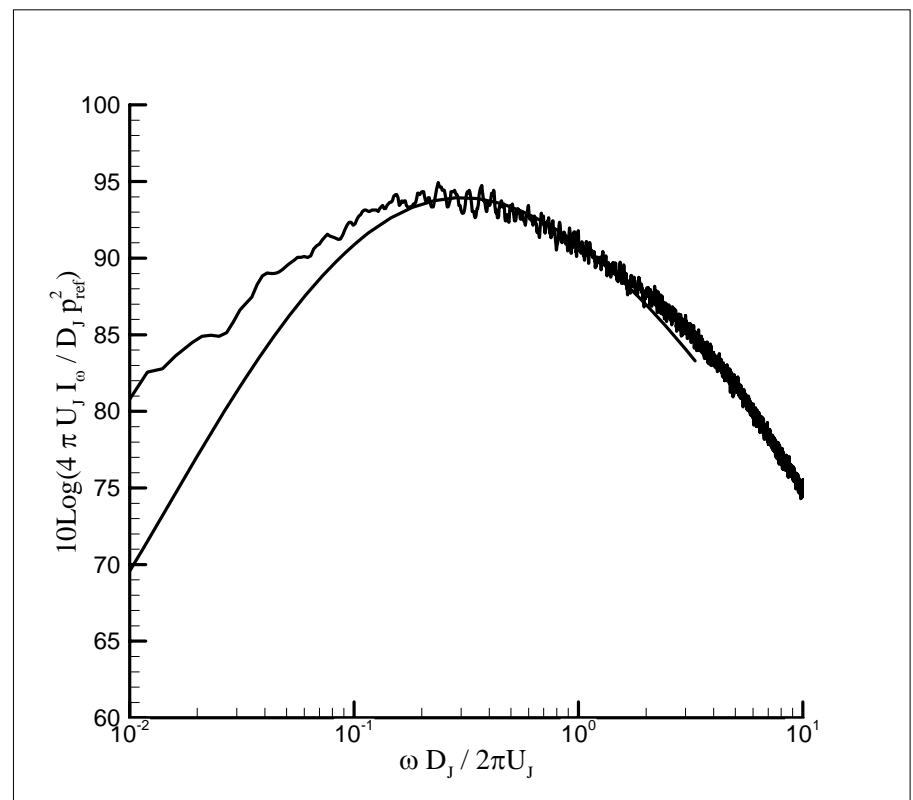

Part b) $M_{J}=0.9, \theta=90^{\circ}$ 


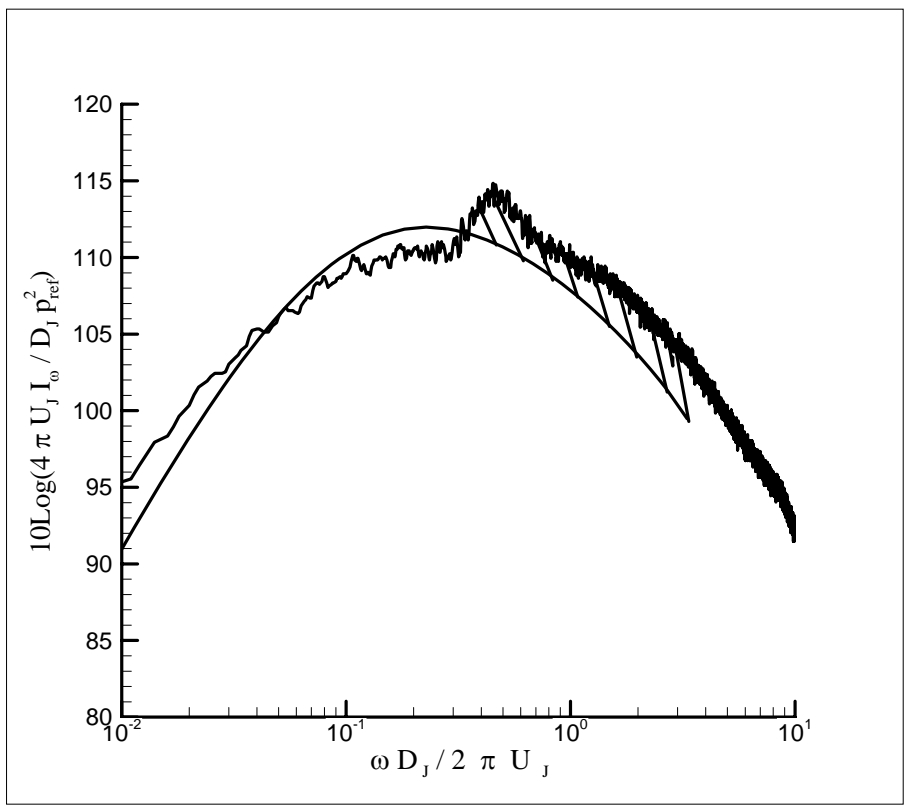

Part c) $M_{J}=1.4, \theta=90^{\circ}$

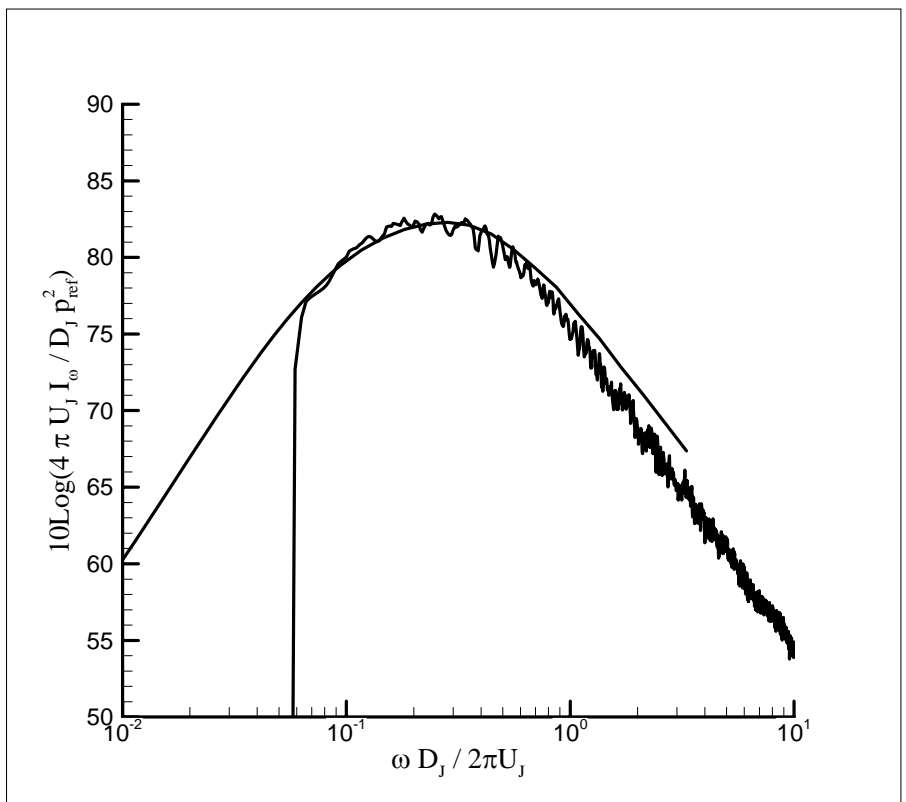

Part d) $M_{J}=0.5 \theta=30^{\circ}$ 


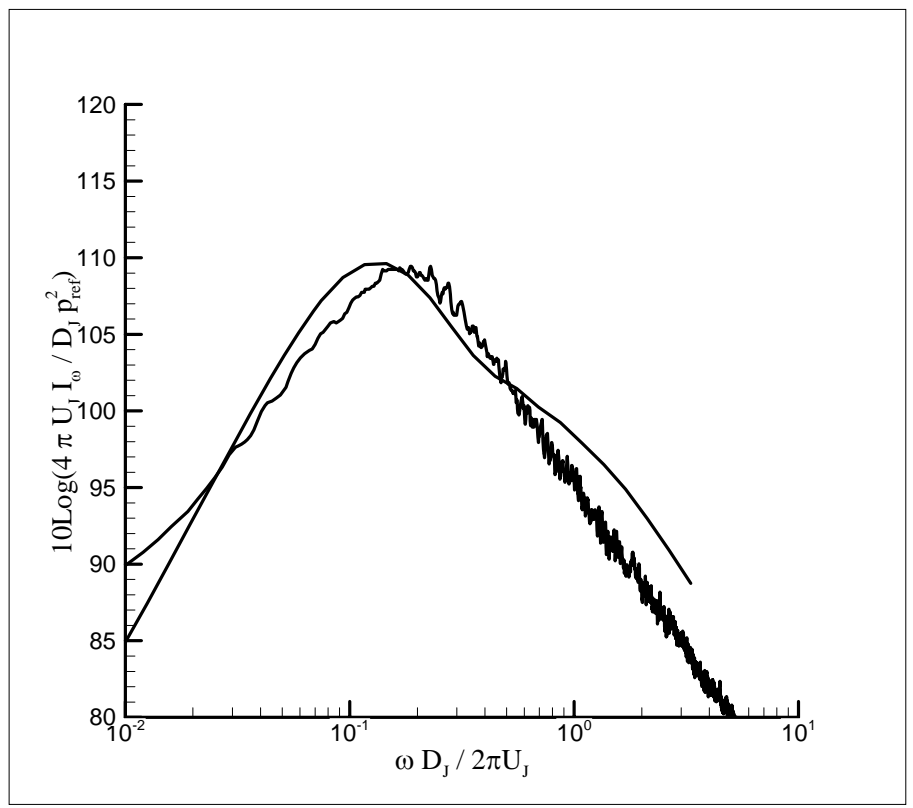

Part e) $M_{J}=0.9, \theta=30^{\circ}$

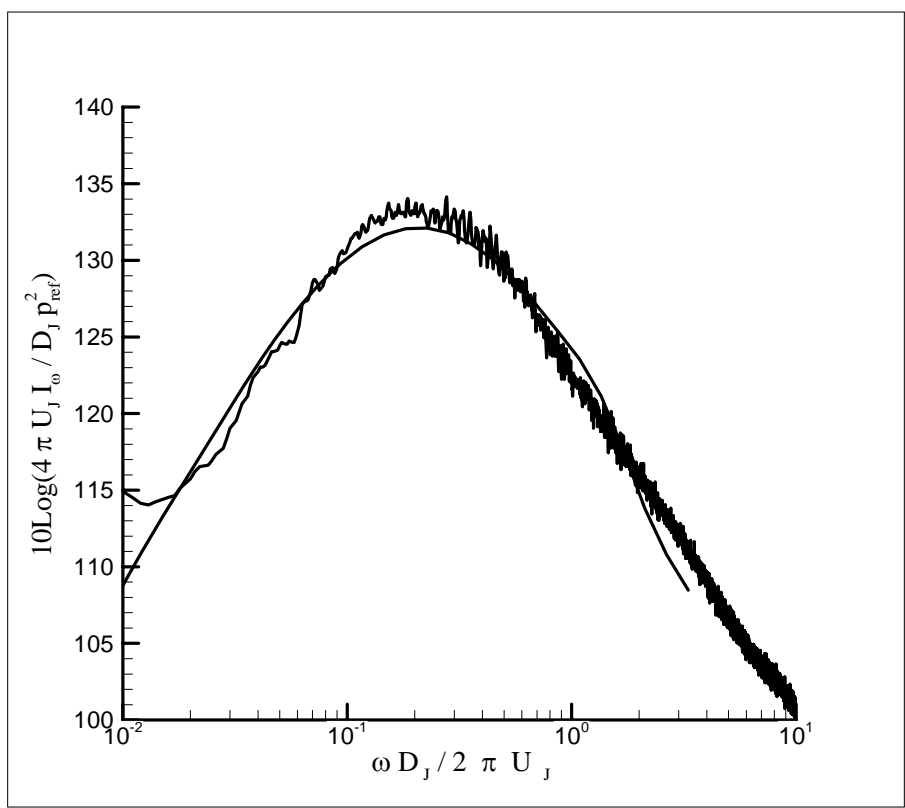

Part f) $M_{J}=1.4 \theta=30^{\circ}$

Figure 6 Comparison of Predictions with data 


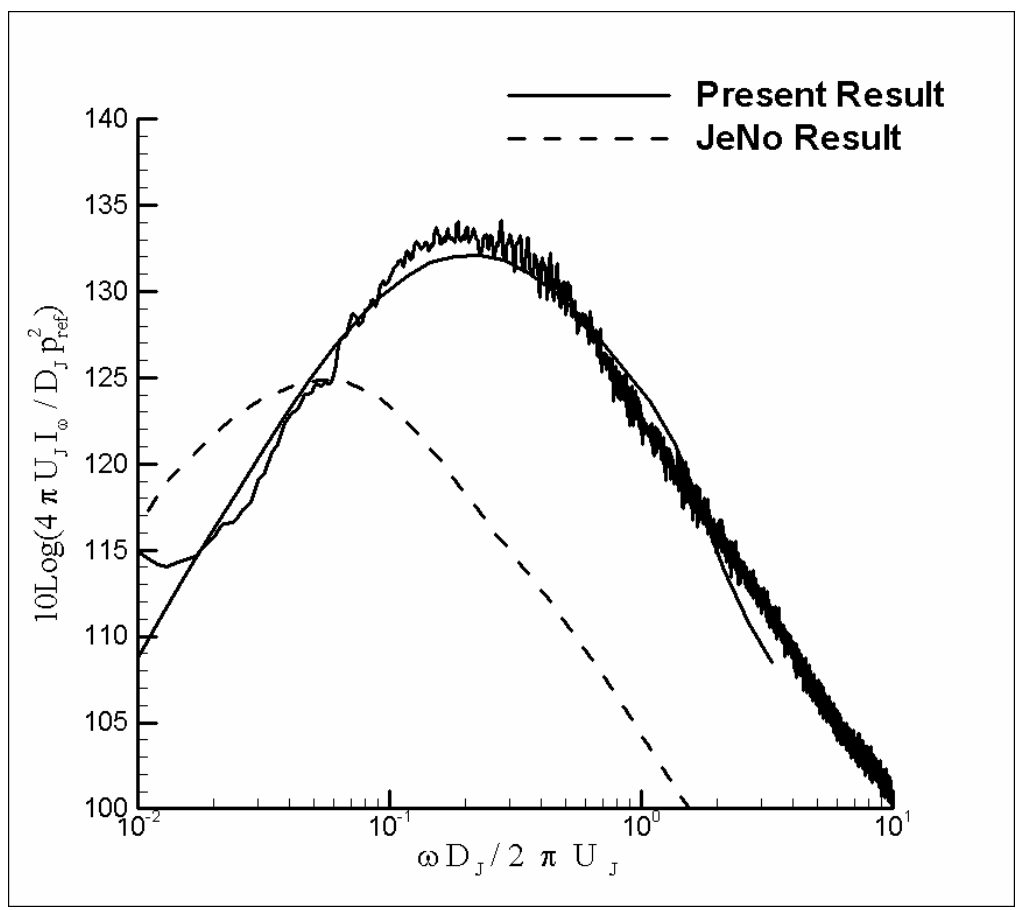

Figure 7. Comparison with best previous results obtained from Glenn JeNo code (Khavaran, Bridges,. \& Georgiadis, N., 2005)
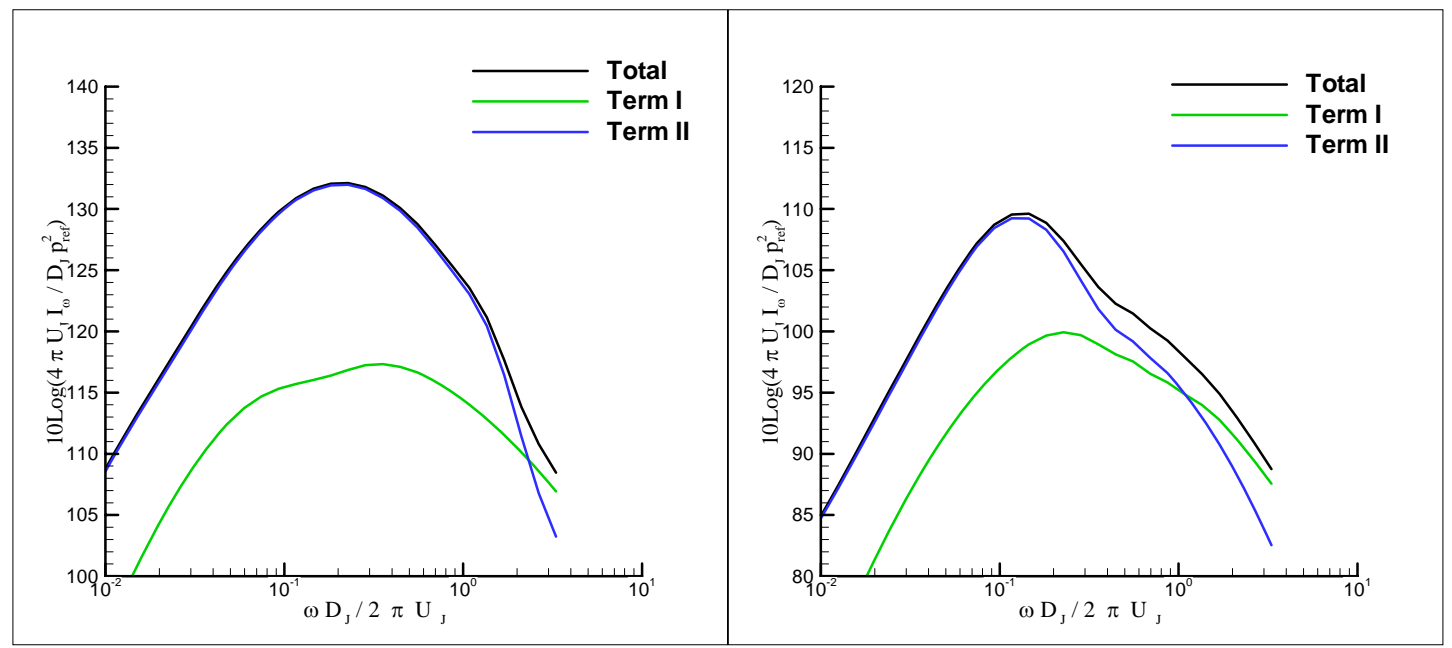

Part (a) $M_{J}=1.4$

Part (b) $M_{J}=0.9$ 


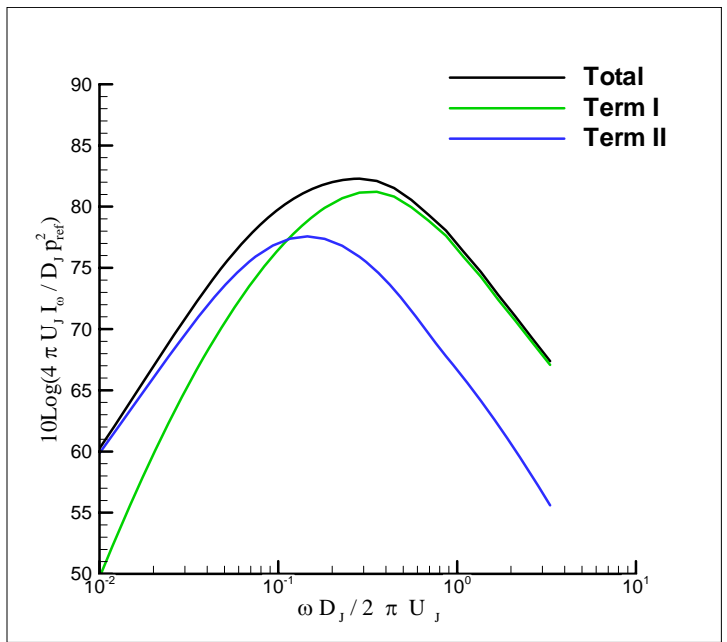

Part (c) $M_{J}=0.5$

Figure 8. Contribution of individual terms to overall spectra at $\theta=30^{\circ}$

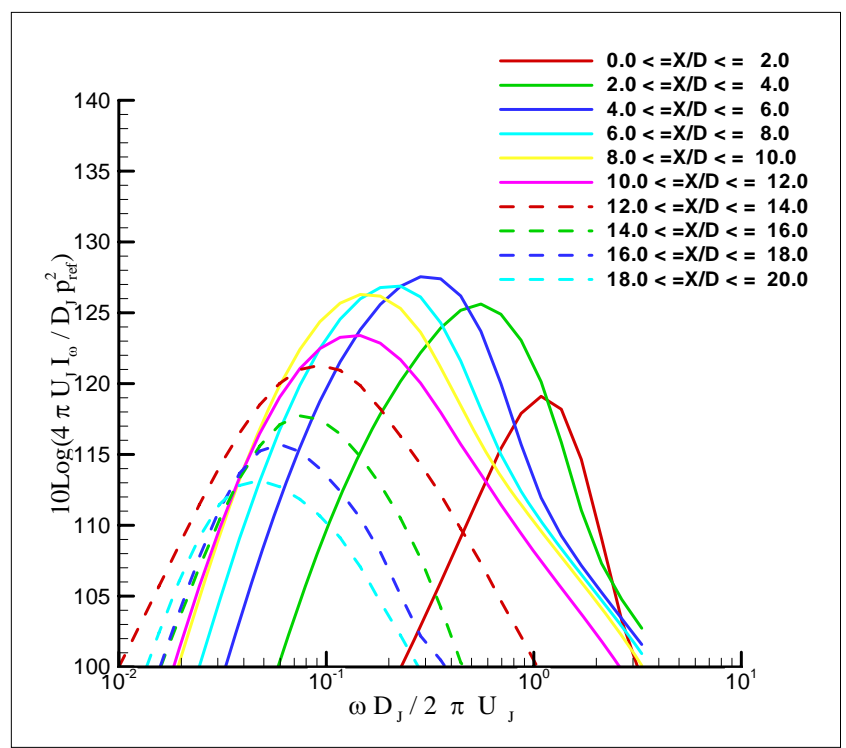

Part a) $\theta=30^{\circ}$ 


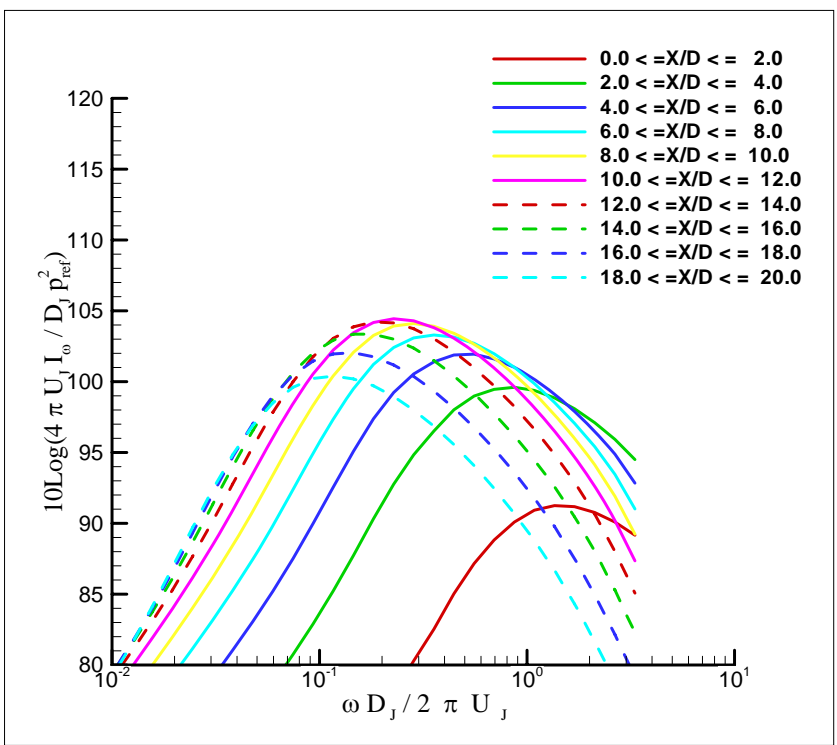

Part b) $\theta=90^{\circ}$

Figure 9 Contribution of individual slices to overall spectrum for $M_{J}=1.4$ jet 